\title{
FUNÇÕES GERADORAS DE MOMENTOS
}

\author{
ANGELA MARIA CASSAVIA JORGE CORRÊA
}

Orientador: Prof. Dr. IZAIAS RANGEL NOGUEIRA

Dissertação apresentada à Escola Superior de Agricultura "Luiz de Queiroz", da Universidade de São Paulo, para obtenção do título de Mestre em Estatística e Experimentação Agronômica.

PIRACICABA

Estado de São Paulo - Brasil

Novembro, 1981 
ii.

A meu marido,

A meu filho,

A meus pais,

OFERECOO. 


\section{AGRADECIMENTOS}

Ao Dr. Izaias Rangel Nogueira, Professor Titular do Departamento de Matemática e Estatística da ESALQ/USP, pela valiosa orientação, incentivos e sugestões durante a elaboração deste trabalho.

Ao Dr. Humberto de Campos, Coordenador do Curso de Estatística ex perimentação Agronômica da ESALQ/USP, pelas sugestões e incenti vos.

Ao Dr. Frederico Pimentel Gomes, Professor Catedrático do Departamento de Matemática e Estatística da ESALQ/USP, pelo incentivo e auxílio dado na elaboração do Summary.

Ao Prof. Renato Soliani, Chefe do Departamento de Matemática da UNIMEP, pela valiosa orientação sobre Variáveis Complexas.

Ao Prof. José Francisco C. Marques, do Departamento de Matemática da UNIMEP, pela valiosa orientação sobre Convergência de Séries.

A meu marido, Coriolano Ferraz Corrêa, pelo apoio e incentivos.

Ao corpo docente do Departamento de Matemática e Estatística da ESALQ/USP, pelos ensinamentos.

A todos que, de uma forma ou de outra, contribuiram para a realização deste trabalho. 


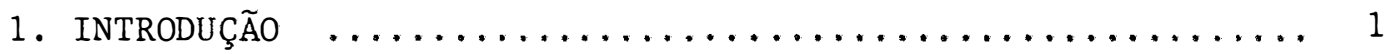

2. REVISÃo de Literatura $\ldots \ldots \ldots \ldots \ldots \ldots \ldots \ldots \ldots \ldots \ldots \ldots \ldots \ldots$

3. FUndAMENTOS TEÓRICOS $\ldots \ldots \ldots \ldots \ldots \ldots \ldots \ldots \ldots \ldots \ldots \ldots \ldots \ldots$

3.1 - Considerações Preliminares $\ldots \ldots \ldots \ldots \ldots \ldots \ldots \ldots$

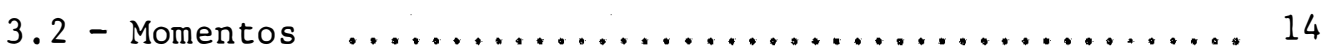

3.2 .1 - Momentos em relação à origem ............ 15

3.2.2 - Momentos conjuntos em relação à origem .... 15

3.2 .3 - Momentos centrados $\ldots \ldots \ldots \ldots \ldots \ldots \ldots \ldots \ldots$

3.2 .4 - Momentos conjuntos em relação à média . ... 17

3.2.5 - Relação geral entre momentos centrados e momentos em relação à origem $\ldots \ldots \ldots \ldots \ldots 17$

3.2.5.1 - Cálculo de alguns momentos pela relação geral entre momentos centrados e momentos em relação à origem 10

3.2 .6 - Momentos absolutos ..................... 20

3.2 .7 - Momentos fatoriais $\ldots \ldots \ldots \ldots \ldots \ldots \ldots \ldots \ldots \ldots \ldots \ldots$

3.3 - Funções Geradoras de Momentos (f.g.m.) ........... 24

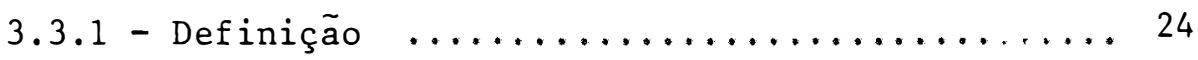

3.3 .2 - Algumas propriedades das f.g.m. ........ 27

3.3 .3 - Funções geradoras de momentos conjuntos ....28 3.3 .3 .1 - Definição $\ldots \ldots \ldots \ldots \ldots \ldots \ldots 28$ 
3.3.3.2 - Algumas propriedades das f.g.m. con juntos $\ldots \ldots \ldots \ldots \ldots \ldots \ldots \ldots \ldots 29$

3.3.3.3 - Funções geradoras de momentos marginais $\ldots \ldots \ldots \ldots \ldots \ldots . \ldots . \ldots 30$

3.3.3.4 - Função geradoras de momentos conjun tos e independência estocástica mütua de $\left(x_{1}, \ldots, x_{k}\right) \ldots . . . . .30$

3.3 .4 - Funções geradoras de momentos fatoriais .... 34

3.3 .4 .1 - Definição $\ldots \ldots \ldots \ldots \ldots \ldots \ldots \ldots$

3.4 - Cálculo da Funçäo Geradora de Momentos e Principuis Momentos, para Algumas Distribuições Especiais de Pro babilidade $\ldots \ldots \ldots \ldots \ldots \ldots \ldots \ldots \ldots \ldots \ldots \ldots . \ldots . \ldots . \ldots$

3.4 .1 - Distribuições discretas $\ldots \ldots \ldots \ldots \ldots \ldots . \ldots 36$

3.4.1.1 - Distribuição uniforme discreta ...36

3.4.1.2 - Distribuição de Bernoul1i ...... 38

3.4.1.3 - Distribuição Binomial ........ 39

3.4.1.4 - Distribuição de Poisson ....... 42

3.4.1.5 - Distribuição geomētrica (ou Pascal) 45

3.4.1.6 - Distribuição binomial negativa ... 47

3.4.1.7 - Distribuição multinomial ...... 50

3.4 .2 - Distribuições continuas $\ldots \ldots \ldots \ldots \ldots \ldots \ldots$

3.4.2.1 - Distribuição retangular ou uniforme 53

3.4.2.2 - Distribuição exponencial (negativa) 55

3.4 .2 .3 - Distribuição gama ......... 57 
Päg.

3.4 .2 .4 - Distribuiçäo beta $\ldots \ldots \ldots \ldots \ldots .59$

3.4.2.5 - Distribuição normal ........6 61

3.4.2.6 - Distribuição de qui-quadrado .... 70

3.4 .2 .7 - Distribuição multinormal ..... 72

3.5 - Fórmula Geral para Obtenção de Momentos nas Distriuui ções Bernoulli, Binomial, Poisson, Geométrica, Binomial Negativa, Normal, Gama e Exponencial (Negativa) 80

3.5 .1 - Förmula recorrente (NOGUEIRA, 1965) ..... 80

3.5 .2 - Distribuição de Bernoulli ........... 83

3.5 .3 - Distribuição binomial ............ 85

3.5 .4 - Distribuição de Poisson ............ 87

3.5 .5 - Distribuição geométrica (Pascal) ........ 89

3.5 .6 - Distribuição binomial negativa ........ 92

3.5 .7 - Distribuição exponencial (negativa) ....... 94

3.5 .8 - Distribuição gama $\ldots \ldots \ldots \ldots \ldots \ldots \ldots \ldots$

3.5 .9 - Distribuição normal .............. 99

3.6 - Técnica da Função Geradora de Momentos ........... 100

3.6 .1 - Descrição da técnica ................ 101

3.6.1.1 - Aplicações da técnica das f.g.m. à obtenção de distribuições conjuntas 102

3.6.2 - Distribuição da soma de V.A. independentes .. 107

3.6.2.1 - Aplicações da técnica da f.g.m. à distribuição da soma de V.A. independentes $\ldots \ldots \ldots \ldots \ldots \ldots \ldots$ 
vii.

Pāg.

3.6.3 - Aplicação da técnica da f.g.m. à obtenção de distribuições limite $\ldots \ldots \ldots \ldots \ldots \ldots \ldots . \ldots$

3.6.3.1 - Função de distribuição 1imite ... 118

3.6.3.2 - Aplicação 1: Obtenção da distribui ção 1 imite da distribuição binomial quando $n$ è grande e $\mathrm{p}$ é pequeno...

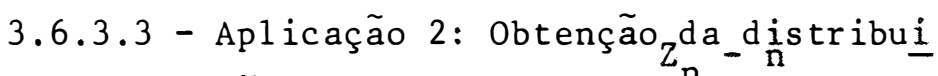
ção 1 imite para $Y_{n}=\frac{{ }_{n}}{\sqrt{2 n}}$ quando n é grande $\ldots \ldots \ldots \ldots \ldots \ldots \ldots$

3.6.3.4 - Aplicação da f.g.m. limite ao tenrema do limite central ........

3.7 - Funções Caracteristicas $\ldots \ldots \ldots \ldots \ldots \ldots \ldots$

3.7 .1 - Definição $\ldots \ldots \ldots \ldots \ldots \ldots \ldots \ldots \ldots \ldots \ldots \ldots \ldots \ldots \ldots \ldots \ldots$

3.7.2 - Obtenção de momentos a partir da função caracteristica $\ldots \ldots \ldots \ldots \ldots \ldots \ldots \ldots \ldots$

3.7.2.1 - Obtenção dos momentos por desenvol vimento em série da função caracte ristica $\ldots . . \ldots \ldots \ldots \ldots \ldots . . . \ldots$

3.7.2.2 - Obtenção dos momentos por derivação sucessiva de $\phi_{X}(t) \quad \ldots \ldots \ldots$

3.7.2.3 - Cálculo de momentos atravēs da fun ção característica: distribuição ga ma $\ldots \ldots \ldots \ldots \ldots \ldots \ldots \ldots$

3.7.3 - Algumas propriedades das funções características $\ldots . . \ldots \ldots \ldots \ldots \ldots \ldots \ldots . . \ldots \ldots$

3.7.4 - Alguns teoremas básicos das funções caracteristicas $\ldots \ldots \ldots \ldots \ldots \ldots \ldots \ldots \ldots \ldots$ 
viii.

Pág.

3.7.4.1 - Teorema da inversão ........ 137

3.7.4.2 - Teorema da unicidade $\ldots \ldots \ldots \ldots 146$

3.7.5 - Funções características para funções de varias variāveis aleatórias ............ 146

3.7 .5 .1 - Definição $\ldots \ldots \ldots \ldots \ldots \ldots \ldots 147$

3.7.5.2 - Propried ades ............. 147

3.7 .5 .3 - Teoremas $\ldots \ldots \ldots \ldots \ldots \ldots \ldots \ldots 148$

3.7.6 - Cálculo das funções características de algumas distribuições especiais de probabilidade

3.7.6.1 - Distribuições discretas ....... 150

3.7 .6 .2 - Distribuições contỉnuas ....... 156

3.7 .7 - o caso da distribuição de Cauchy ........ 162

3.7.7.1 - Distribuição de Cauchy ....... 162

3.7.7.2 - Verificação de que a função gerado ra de momentos para a distribuição de Cauchy não existe .......... 163

3.7.7.3 - Obtenção da função caracterỉstica de uma distribuição de Cauchy ...

4. RESULTADOS E DISCUSSÁ $\ldots \ldots \ldots \ldots \ldots \ldots \ldots \ldots \ldots \ldots \ldots \ldots$

4.1 - Definiçäo de Momentos .................... 169

4.2 - Funções Geradoras de Momentos ............... 170

4.3 - Generalização da Förmula Recorrente (NOGUEIRA, 1 ,65: 171

4.4 - Técnica da Função Geradora de Momentos .......... 174

4.5 - Momentos da Distribuição Normal ............. 176

4.6 - Teorema da Inversäo $\ldots \ldots \ldots \ldots \ldots \ldots \ldots \ldots \ldots \ldots \ldots$ 
Pāg .

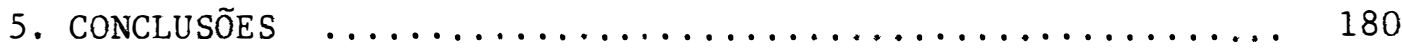

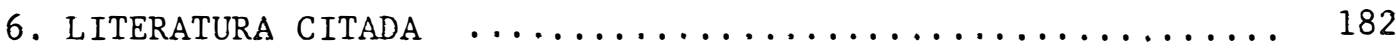

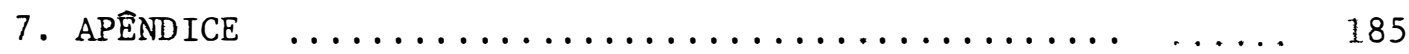


Autora:

Orientador:
Angela Maria Cassavia Jorge Corrêa

Dr. Izaias Rangel Nogueira

\section{$R E S \cup M O$}

Para se caracterizar uma distribuição de probabilida de, é de fundamental importância o conhecimento de seus principais momentos, principalmente o primeiro momento em relação à origem (mé dia), o segundo momento em relação à média (variância), e o terceiro e o quarto momentos em relação à média, utilizados na caracterização da assimetria e da curtose, respectivamente, de uma distribui ção.

Esta dissertação pretende ser um estudo abrangente so bre os momentos de uma distribuição, as funções geradoras de momentos e funções características, förmulas recorrentes para o cálculo de momentos de ordem superior ao segundo, aplicações da técnica da função geradora de momentos e do teorema da Inversão. Faz-se, para tanto, um trabalho de revisão bibliográfica e apresenta-se, de forma didática, o que se considera de maior importância, bem como se fornecem contribuições pröprias, particularmente para momentos de ordem superior ao segundo. Com essa finalidade: 
- definem-se momentos usuais, momentos conjuntos, absolutos e fatoriais e apresenta-se uma importante relação geral entre momentos centrados e momentos em relação à origem;

- define-se função geradora de momentos, e apresentam-se suas principais propriedades;

- obtém-se as funções geradoras de momentos, e calculam-se os principais momentos para algumas distribuições especiais de probabi lid ade;

- apresenta-se a função geradora de momentos fatorial e aplica-se a mesma às distribuições binomial e Poisson;

- estuda-se a fórmula recorrente (NOGUEIRA, 1965) para momentos de ordem superior à segunda, e generaliza-se o seu resultado pa ra algumas distribuiçōes discretas e contínuas;

- apresenta-se a técnica da função geradora de momentos e suas aplicações fundamentais, como obtenção de distribuições conjuntas, de soma de variáveis aleatórias independentes e distribuições limite;

- define-se função característica, obtêm-se momentos a partir de funçöes características, apresentam-se suas propriedades e teore mas fundamentais;

- aplica-se o teorema da inversão para obtenção de funções de densidade;

- obtém-se a função característica de algumas distribuições es peciais de probabilidade; 
xii.

- apresenta-se a distribuição de Cauchy como exemplo de uma distribuição que não possui funçăo geradora de momentos e obtém-se a sua função característica.

Por ser uma dissertação estritamente teórica, é uma contribuição didática e bibliográfica sobre o tema desenvolvido. 
xiii.

MOMENT GENERATING FUNCTIONS

Candidate: Angela Maria Cassavia Jorge Corrêa

Adviser: Dr. Izaias Rangel Nogueira

$S U M M A R Y$

This dissertation is mostly a review of literature on moments of probability distributions, moment generating functions and characteristic functions, as well as on recurrence formulas for moment calculation. There are, however, a few author's contributions, specially in connection of moments of higher orders.

Having this in view, the author shows the main topics of the theory, hoping that it will favor the teaching and studying of the subject. 
Entre as funções geradoras de momentos, destaca-se a função $m_{x}(t)=E\left[e^{t x}\right]$, que é denominada simplesmente função geradora de momentos e a função $\phi_{X}(t)=E\left[e^{i t x}\right]$, denominada função ca racterística. Alguns tratados de Estatística mais avançados apresen tam essas funções geradoras, dando ênfase especial à função caracte ristica. Outros, limitam-se ao estudo da função geradora de momentos e à aplicação de sua técnica, apenas citando as funções caracte rísticas.

Além das funções geradoras, têm-se também förmulas recorrentes, que permitem obter, de modo rápido e fácil, os momentos de ordem superior ao segundo.

A finalidade desta dissertação é a apresentação, em um só conjunto, de um estudo detalhado e ordenado sobre a função ge 
radora de momentos e a função característica, com ênfase em alguns tópicos e aplicações, tais como: momentos, funções geradoras de momentos e funções características das distribuições de uso mais corrente em Estatística, förmulas recorrentes, aplicações da técnicada função geradora de momentos e de alguns teoremas fundamentais, como o teorema da inversão.

Acredita-se que este trabalho é uma contribuição didática para alunos do Curso de Pós-Graduação em Estatística e Experimentação Agronômica da ESALQ/USP, pela ordenação didática dos tópicos desenvolvidos e ampliados com contribuições próprias, além de bibliográfica, pois a busca em livros é muito desordenada.

Espera-se que esta dissertação venha a ser $\vec{u}$ ill para aqueles estudantes que se iniciam no estudo de funções geradoras de momentos, e para tanto procura-se tornar o texto o mais claro e sim ples possível. 


\section{REVISAOO DE LITERATURA}

Funções geradoras de momentos e funções característi cas foram originariamente desenvolvidas como um instrumento para a solução de problemas em Teoria de Probabilidades e em Estatística Matemática. o seu estudo, e o de suas importantes aplicações, vem sendo feito hả algum tempo e por diversos autores.

Segundo TATON (1964), vários matemáticos famosos jä se dedicaram ao estudo das funções geradoras de momentos. LAPLACE, em 1812, em sua obra Théorie Analytique des Probabilités, apresentou uma teoria das funções geradoras de momentos, também chamadas funções geratrizes de momentos. CAUCHY, em 1853, definiu com precisão a função característica $\int e^{i t x} f(x) d x$, e a aplicou à função atualmente conhecida como função de densidade de probabilidade de Cauchy,

$$
f(x)=\frac{1}{\pi} \cdot\left[\frac{1}{1+x^{2}}\right] .
$$


A introdução da teoria das funções caracteristicas dentro da Estatística, particularmente à Teoria de Probabilidades, deve-se a H. Poincaré e P. Lévy. Poincaré, em 1912, deu a conhecimento a definição e o nome da função característica, porém a considerando como $E\left[e^{t x}\right]$. P. Lévy em 1925, apresentou a prova do primeiro teorema do limite e, de forma até certo ponto menos geral, o teorema da inversão. Posteriormente, LEVY e CRAMER (1937) demonstra ram simultaneamente a forma generalizada, hoje conhecida, do teorema da inversão.

CRAMER (1955) estuda funções geradoras de momentos, particularmente as do tipo $E\left(e^{t x}\right)$ para funções de uma variāvel alea tória, efetuando apenas citação rāpida da definição de momentos para distribuições em duas dimensões. Ao estudar funções geradoras do tipo $E\left(e^{t x}\right)$, afirma, sem demonstrar, que a existência da função geradora $E\left(e^{t x}\right)$, ou de uma sequência de momentos, determina a correspondente distribuição unicamente e, também, que a função geradorade momentos de uma soma de variáveis aleatórias independentes é o produto das funções geradoras de momentos dos termos. Apresenta as fun ções geradoras de momentos para as distribuições Binomial, Poisson e Normal, e a seguinte expressão para o cálculo de momentos de ordem par para esta ūltima:

$$
\begin{aligned}
& \text { Se } x \cap N(0,1) \text {, então } \mu_{2 n}=1,3 \ldots(2 n-1) \\
& \text { Se } x \cap N\left(m, \sigma^{2}\right) \text {, então } \mu_{2 n}=1 \cdot 3 \ldots(2 n-1) \cdot \sigma^{2 n} \text {. } \\
& \text { Afirma também que as funções características têm im- }
\end{aligned}
$$
portantes aplicações na teoria geral das distribuições, porëm não 
as estuda, sob a alegação da não necessidade de se introduzir variā veis complexas em grande maioria das aplicações das funções geradoras de momentos.

WILKS (1962), utilizando conceitos avançados de Mate mática, como a Teoria da Integração de Lebesque-Stieltjes, efetua um estudo geral sobre Cálculo de Probabilidades, inclusive sobre fun ções geradoras de momentos e funções características.

NOGUEIRA (1965) apresenta uma fórmula geral para a obtenção de momentos de ordem superior ao segundo, e verifica a sua validade para as distribuições Binomial, Poisson e Normal.

NOGUEIRA (1968) desenvolve um trabalho muito interes sante sobre a aplicação de matrizes ao estudo da distribuição multi normal, desde a obtenção da função de densidade multinormal (para vạ riáveis $x_{i}$ normais de média 0 e variância $\sigma_{i}^{2}$ ) em forma matricial, até o cálculo dos momentos da distribuição, atravēs da forma matricial da função geradora de momentos. Apresenta, como caso particular, a distribuição binormal.

PIEDRABUENA (1968), devido a uma anomalia relativa à distribuição de Poisson, em contagens de leveduras, chega a uma dis tribuição binomial que não é mais que o modelo de GREENWOOD e YULE, mas por diferente caminho e com o conceito de interferência. Efetua o desenvolvimento dos momentos sobre esse modelo, e verifica que a fórmula proposta por NOGUEIRA (1965) se aplica ao mesmo.

LUKACS (1970) desenvolve uma monografia ampla sobre funções características, baseando-se para tanto em literatura em 
grande parte de origem russa e francesa, o que reveste de especial importância a obra, pois esta parte da literatura muitas vezes não é facilmente acessível, e seu uso dificultado pela barreira idiomätica. Apresenta o estudo de funções características por seu interes se intrínseco matemático, e não se dedica às suas aplicações, desen volvendo definições, propriedades, teoremas fundamentais e os mais recentes resultados sobre o assunto.

HOOG e CRAIG (1970) apresentam um estudo das funções geradoras de momentos do tipo $E\left(e^{t x}\right)$ para funções de uma variāvel aleatória (e definem funções geradoras de momentos para distribuições conjuntas), através do cálculo (ou indicação) das funções gera doras de momentos de algumas distribuições especiais de probabilidade. Através de teoremas, aplicam a função geradora de momentos pa ra obtenção de distribuições da soma de variāveis aleatörias independentes. Exemplificam como obter distribuições conjuntas através do conhecimento de funções geradoras de momentos. Definem distribui ções limite, tecem considerações sobre funções geradoras de momentos limite e aplicações. Apresentam um caso especial do teorema do limite central e o demonstram com auxílio de funções geradoras de momentos limite.

TORANZOS (1971) define função geradora de momentos (chama-a função característica), e função característica (chama-a função característica complexa), além de citar a função característica logarítmica. Calcula as funções geradoras de momentos das dis- 
tribuições binomial e normal padrão, e apresenta, para esta ủltima, a seguinte fórmula para obtenção de momentos de ordem par:

$$
\mu_{2 n}=\frac{(2 n) !}{n ! 2^{n}} .
$$

Efetua, também, o cálculo das funções características para as distribuições Binomial e Normal Padrão, e cita alguns teoremas sobre aplicações das funções geradoras de momentos na obtenção de distribuições. Realiza um estudo rápido, em forma algébrica e matricial, que o conduz à função geradora de momentos de uma distribuição normal multivariada.

ROUSSAS (1973) desenvolve um estudo sobre funções ca racterísticas para funções de uma variável aleatória, generalizando-o para distribuições conjuntas e, no decorrer desse trabalho, def $\underline{i}$ ne rapidamente funções geradoras de momentos do tipo $E\left(e^{t x}\right)$, e fato riais. Em um capitulo especial sobre momentos de algumas distribuições, demonstra que, em uma distribuição normal padronizada, os momentos de ordem par são dados por:

$$
\mu_{2 n}=\frac{(2 n) !}{2^{n}(n !)} \text {. }
$$

Aplica as funções características para obtenção de algumas distribuições de somas de variáveis aleatórias independentes, e obtēm, através de desenvolvimento matricial, a expressão da função característica de uma distribuição multinormal. Calcula funções caracterís ticas, geradoras de momentos e fatoriais de algumas distribuições discretas e contínuas, e apresenta, sem demonstração, os teoremas da Inversão e da Unicidade. 
MOOD et alii (1974) destacam, em sua obra, a função geradora de momentos do tipo $E\left(e^{t x}\right)$, e sua importante aplicação para obtenção de funções de variáveis aleatōrias: a técnica da função geradora de momentos. Definem momentos, momentos fatoriais, funções geradoras de momentos e de momentos fatoriais. Ao estudarem algumas distribuições especiais de probabilidade, apresentam suas respectivas funções geradoras de momentos. Dedicam atenção ao teorema do $1 \underline{i}$ mite central, em restrição particular, e o demonstram com utilização da técnica da função geradora de momentos e de distribuições 1 i mite.

CRAMER (1974) apresenta um estudo profundo de Estatística Matemática, reunindo em sua obra duas linhas do desenvolvimento clássico do Cálculo de Probabilidades, a Francesa e a Russa, utilizando teoria matemática pura, particularmente a Teoria da Medi da, de Lebesque. Estuda com profundidade a função característica e suas aplicações. Salienta-se que a primeira edição dessa obra data de 1946.

KENDALL e STUART (1977), definindo momentos, apresen tam importante relação geral entre momentos centrados e em relação à origem. Também definem momentos absolutos e fatoriais, funções ge radoras de momentos, funções características e funções geradoras de momentos fatoriais. Não dão ênfase em seu trabalho às funções geradoras de momentos, mas, sim, às funções características. Apresentam o teorema da inversão, sua dedução e algumas aplicações, e, ao estụ darem algumas distribuições especiais de probabilidade, calculam as 
respectivas funções características. Ao analisarem as distribuições Binomial e Poisson, fazem referência à fórmula recorrente para cālculo de momentos de ordem superior ao segundo (a partir de funções características), e, ao estudarem a distribuição normal, apresentam a seguinte fórmula para cálculo de momentos de ordem par:

$$
\mu_{2 n}=\frac{(2 n) !}{2^{n} \cdot n !} \sigma^{2 n}, \quad n \geq 1 .
$$

Utilizam o método das funções características para obtenção de distribuições de somas, e particularmente de $\bar{x}$. Apresentam estudo matricial da distribuição normal multivariada, e sua respectiva função característica.

SPIEGEL (1978) apresenta, resumidamente, e em forma de definições, momentos, funções geradoras de momentos e funções cá racterísticas. Para algumas distribuições especiais de probabilidade, faz referência às suas funções geradoras de momentos e/ou às suas funções características.

HOEL (1980) realiza um estudo suscinto sobre momentos, funções geradoras de momentos, e algumas aplicações relativas à obtenção de distribuições.

CRUZ (1980) apresenta o cálculo dos momentos ordinärios (e centrais), de uma distribuição de frequências com intervalos de classe de amplitude constante, através da utilização dos momentos fatoriais. 
10.

\section{FUNDAMENTOS TEOORICOS}

\section{1 - Considerações Preliminares}

Como este trabalho tem por objetivo uma revisão bibliogräfica e ordenação didática a respeito de funções geradoras de momentos, è conveniente introduzir alguns conceitos elementares da Teoria Estatística que são frequentemente utilizados.

\section{- Esperança Matemātica}

O conceito de esperança matemática é de grande impor tância em Probabilidade e Estatística. E também denominado valor es perado ou média.

Define-se esperança matemática de uma variável aleatória discreta $\mathrm{X}$, com função de probabilidade $\mathrm{f}_{\mathrm{X}}(\mathrm{x})$, como

$$
E(X)=\sum_{X} x f_{X}(x),
$$

que existe se, e somente se, essa série for convergente. Define-se a esperança matemática de uma V.A. contínua $X$, com função densidade de probabilidade $f_{X}(x)$, como 
11.

$$
E(X)=\int_{-\infty}^{+\infty} x f_{X}(x) d x
$$

que existe se, e somente se, essa integral é convergente.

Se $X$ é uma V.A., e $Y=g(X)$ também o é, tem-se que

$$
\begin{aligned}
& E[g(X)]=\sum_{X} g(x) f_{X}(x) \quad, \text { se } X \text { é V.A. discreta, e } \\
& E[g(X)]=\int_{-\infty}^{+\infty} g(x) f_{X}(x) d x, \text { se } X \text { è V.A. continua, }
\end{aligned}
$$

que existem se, e somente se, a série e a integral convergirem.

Alguns tratados de Estatística Matemātica apresentam o conceito de esperança matemática com um maior rigor matemático,utilizando a integral de Stieltjes nessa definição. Conceituam esperança matemätica de uma V.A. X e de uma V.A. $Y=g(X)$, como:

$$
E(X)=\int_{-\infty}^{+\infty} x d F \quad \text { e } \quad E[g(X)]=\int_{-\infty}^{+\infty} g(X) d F
$$

que existem se, e somente se, essas integrais forem convergentes.

A vantagem da utilização da integral de Stieltjes,na definição de esperança matemätica, bem como em outros conceitos, $\vec{e}$ que ela evita a necessidade de se exporem todas as definições e teo remas duplamente. Assim, se $F(x)$ è uma função de distribuição com função de densidade de probabilidade continua, a integral de Stielt jes torna-se uma integral comum,

$$
\int_{-\infty}^{+\infty} x f_{X}(x) d x, \text { ou }, \int_{-\infty}^{+\infty} g(x) f_{X}(x) d x,
$$

incluindo, pois, esta ültima, um caso particular; e, se $F(x) \bar{e}$ uma função de distribuição de V.A. discreta, a integral de Stieltjes cor responde ao somatório

$$
\sum_{X} \mathrm{x}_{\mathrm{X}}(\mathrm{x}), \text { ou, } \sum_{\mathrm{X}} \mathrm{g}(\mathrm{x}) \mathrm{f}_{\mathrm{X}}(\mathrm{x}),
$$

tambēm como um caso particular. 
- Alguns Teoremas Sobre Esperança Matemātica

$$
\begin{aligned}
& E(K X)=K E(X), \quad K \text { constante } \\
& E(X+Y)=E(X)+E(Y), \quad X \text { e } Y \text { V.A. } \\
& E(X . Y)=E(X) . E(Y), X \text { e Y V.A. independentes }
\end{aligned}
$$

- A Variäncia e o Desvio Padrão

$$
\text { Define-se variância de uma distribuição de V.A. X co }
$$

mo

$$
\operatorname{Var}(X)=\sigma^{2}(X)=E\left[(X-m)^{2}\right],
$$

e desvio padrão como

$$
\sigma(X)=\sqrt{\operatorname{Var}(X)}=\sqrt{E[(X-m)]^{2}} .
$$

Se $X$ é uma V.A. discreta com função de probabilidade $f_{X}(x)$, a variância de $X \bar{e}$ dada por:

$$
\begin{aligned}
& \sigma^{2}(X)=\operatorname{Var}(X)=E\left[(X-m)^{2}\right]=\sum_{j}\left(x_{j}-m\right)^{2} f_{X}\left(x_{j}\right) \cdot \\
& E \text {, se } X \ddot{e} \text { uma V.A. contínua, com função densidade de }
\end{aligned}
$$
probabilidade $\mathrm{f}_{\mathrm{X}}(\mathrm{x})$,

$$
\sigma^{2}(X)=\operatorname{Var}(X)=E\left[(X-m)^{2}\right]=\int_{-\infty}^{+\infty}(x-m)^{2} f_{X}(x) d x .
$$


- Alguns Teoremas sobre a Variância

$$
\begin{aligned}
& \sigma^{2}(X)=E\left[(X-m)^{2}\right]=E\left(X^{2}\right)-[E(X)]^{2} \\
& \operatorname{Var}(K X)=K^{2} \operatorname{Var}(X), K \text { constante } \\
& \operatorname{Var}(X+Y)=\operatorname{Var}(X)+\operatorname{Var}(Y), \quad X \text { e } Y \text { V.A. independentes }
\end{aligned}
$$

- Funções de Vārias Variāveis Aleatōrias

$$
\text { Define-se esperança matemática da função }
$$

$$
g_{x_{1}}, \ldots, x_{K}\left(x_{1}, \ldots, x_{K}\right) \text { ou } g_{x}(x),
$$

da variável aleatória $\left(X_{1}, x_{2}, \ldots, X_{K}\right)$, ou do vetor aleató: io $X=\left(X_{1}, \ldots, X_{K}\right)^{\prime}$, respectivamente, como sendo

$$
E\left[g_{X_{1}}, \ldots, x_{K}\left(x_{1}, \ldots, x_{k}\right)\right]={ }_{x_{1}, \ldots, x_{K}} g_{X_{1}}, \ldots, x_{K}\left(x_{1}, \ldots, x_{k}\right) f_{x_{1}}, \ldots, x_{K}\left(x_{1}, \ldots, x_{K}\right)
$$

se a variāvel aleatōria $\left(X_{1}, \ldots, X_{K}\right)$ é discreta, e

$E\left[8_{x_{1}}, \ldots, x_{k}\left(x_{1}, \ldots, x_{k}\right)\right]=\int_{-\infty}^{+\infty} \ldots \int_{-\infty}^{+\infty} 8_{x_{1}}, \ldots, x_{k}\left(x_{1}, \ldots, x_{K}\right) f_{x_{1}}, \ldots, x_{k}\left(x_{1}, \ldots, x_{K}\right) d x_{1} \ldots d x_{k}$

se a variável aleatōria $\left(\mathrm{X}_{1}, \ldots, \mathrm{X}_{\mathrm{K}}\right)$ è contínua.

A existência das esperanças, seja para o caso de uma ou várias variáveis aleatórias, depende da existência dos somatórios (caso discreto), ou das integrais (caso contínuo).

$$
\begin{aligned}
& \text { Se } g_{X_{1}, \ldots, x_{K}\left(x_{1}, \ldots, x_{K}\right)=x_{i}} \\
& \qquad\left[g_{x_{1}}, \ldots, x_{K}\left(x_{1}, \ldots, x_{K}\right)\right]=E\left[x_{i}\right]=m_{x_{i}}
\end{aligned}
$$


Essa igualdade tanto pode ser provada para o casodi $\underline{s}$ creto como para o caso contínuo. Apresenta-se neste trabalho a prova para o caso contínuo.

$$
\begin{aligned}
E\left[g_{X_{1}}, \ldots x_{k}\left(x_{1}, \ldots, x_{k}\right)\right] & =\int_{-\infty}^{+\infty} \ldots \int_{-\infty}^{+\infty} x_{i} f_{x_{1}}, \ldots, x_{k}\left(x_{1}, \ldots, x_{k}\right) d x_{1}, \ldots, d x_{K} \\
& =\int_{-\infty}^{+\infty} x_{i} f_{x_{i}}\left(x_{i}\right) d x_{i} \\
& =E\left[x_{i}\right]=m_{X_{i}}
\end{aligned}
$$

onde $\mathrm{f}_{\mathrm{x}_{i}}\left(\mathrm{x}_{\mathrm{i}}\right)$ é a densidade marginal de $\mathrm{x}_{i}$, obtida da densidade con junta, ou seja:

$$
\begin{aligned}
& f_{x_{i}}\left(x_{i}\right)=\int_{-\infty}^{+\infty} \ldots \int_{-\infty}^{+\infty} f_{x_{1}}, \ldots, x_{K}\left(x_{1}, \ldots, x_{K}\right) d x_{1}, \ldots, d x_{i-1}, d x_{i+1}, \ldots, d x_{K} \\
& \text { De modo análogo, pode-se verificar que, se } \\
& g_{X_{1}}, \ldots, x_{K}\left(x_{1}, \ldots, x_{K}\right)=E\left(x_{i}^{2}\right)-\left[E\left(x_{i}\right)\right]^{2} \\
& E\left[g_{X_{1}}, \ldots, x_{K}\left(x_{1}, \ldots, x_{K}\right)\right]=E\left(X_{i}^{2}\right)-\left[E\left(X_{i}\right)\right]^{2}=\operatorname{Var}\left(X_{i}\right)
\end{aligned}
$$

\section{2 - Momentos}

Antes de definir-se função geradora de momentos è ne cessārio tecer algumas considerações sobre momentos. 


\section{2 .1 - Momentos em relaçăo à origem}

Define-se o momento de ordem $\underline{r}$ de uma V.A. em relação à origem, e nota-se por $\mu_{r}^{\prime}$ a

$$
\mu_{r}^{\prime}=E\left(X^{r}\right), \quad r=0,1,2, \ldots
$$

se essa esperança existe, ou seja,

$$
\mu_{r}^{\prime}=E\left(x^{r}\right)=\sum_{j} x_{j}^{r} f\left(x_{j}\right),
$$

se $x$ é V.A. discreta, e,

$$
\mu_{r}^{\prime}=E\left(X^{r}\right)=\int_{-\infty}^{+\infty} x^{r} f_{X}(x) d x
$$

se $\mathrm{x} \overrightarrow{\mathrm{e}} \mathrm{V} \cdot \mathrm{A}$. continua.

Se $r=1$, tem-se o primeiro momento em relação à origem, que é a média de $X$,

$$
\mu_{1}^{\prime}=E(X)=m_{X}
$$

Se $\mu_{r}^{\prime}$ existe, também existe $\mu_{s}^{\prime}$ para $s<r$, e se $\mu_{r}^{\prime}$ não existe, nem $\mu_{s}^{\prime}$ para $s>r$.

\subsection{2 - Momentos conjuntos em relação à origem}

Define-se momentos conjuntos em relação à origem a

$$
\begin{aligned}
& E\left[\begin{array}{cccc}
{ }^{r_{1}} & \mathrm{r}^{\mathrm{r}_{2}} & \mathrm{x}_{1}{ }^{\mathrm{r}_{\mathrm{K}}} \\
\mathrm{x}_{2} & \cdots & \mathrm{x}_{\mathrm{K}}
\end{array}\right] \text {, com } \mathrm{r}_{\mathrm{i}} \geq 0 \\
& i=1,2, \ldots, k
\end{aligned}
$$




\subsection{3 - Momentos centrados}

o momento de ordem $\underline{r}$ de uma V.A., centrado em rela-

ção a ạ, è

$$
\mu_{r}(a)=E\left[(X-a)^{r}\right] \quad, r=0,1,2,3, \ldots
$$

Se $a=m_{X}$, tem-se o momento de ordem $\underline{r}$ centrado em $r \underline{e}$ lação à média da distribuição, denotado por $\mu_{r}$,

$$
\mu_{r}=E\left[\left(x-m_{x}\right)^{r}\right] \text {, }
$$

ou seja,

$$
u_{r}=E\left[\left(X-m_{X}\right)^{r}\right]=\sum_{j}\left(x_{j}-m_{X}\right)^{r} f_{X}\left(x_{j}\right)
$$

se $x$ ë V.A. discreta, e

$$
\mu_{r}=E\left[\left(X-m_{X}\right)^{r}\right]=\int_{-\infty}^{+\infty}\left(x-m_{X}\right)^{r} f_{X}(x) d x
$$

se $\mathrm{x}$ ë V.A. continua.

$$
\text { Define-se } \mu_{0}^{\prime}=\mu_{0}=\int_{-\infty}^{+\infty} d P=1, \operatorname{com} d P=f_{X}(x) d x \text {. }
$$

Observa-se que:

$$
\begin{array}{lll}
\mu_{1}=0 & \text { pois } \mu_{1}=E\left(X-m_{X}\right)=E(x)-m_{X}=m_{X}-m_{X}=0 \\
\mu_{2}=\sigma^{2}(x), & \text { pois } \mu_{2}=E\left[\left(X-m_{X}\right)^{2}\right]=E(X)-[E(x)]^{2}
\end{array}
$$

e, tambēm, que todos os momentos de ordem impar de $\mathrm{X}$ em relação a $\mathrm{m}_{X}$, se existem, são nulos se a função de densidade de probabilidade (ou 
função de probabilidade) de $X$ é simétrica em relação à média da dis tribuição.

\subsection{4 - Momentos conjuntos em relação à mēdia}

Define-se momento conjunto em relação à média por

$$
\begin{aligned}
& E\left[\left(x_{1}-m_{x_{1}}\right)^{r_{I}} \ldots\left(x_{K}-m_{x k}\right)^{r_{K}}\right], r_{i} \geq 0 \\
& i=1,2, \ldots, k
\end{aligned}
$$

Em particular, se $r_{i}=r_{j}=1$, e os demais valoresde r são nulos, o momento conjunto em relação à média torna-se a covariância entre $x_{i}$ e $x_{j}$, ou seja,

$$
\operatorname{Cov}\left(x_{i}, x_{j}\right)=E\left[\left(x_{i}-m_{x_{i}}\right)\left(x_{j}-m_{x_{j}}\right)\right]
$$

\subsection{5 - Relação geral entre momentos centrados e momentos em relação à origem}

E possivel determinar momentos centrados (em relação à média), a partir dos momentos em relação à origem, utilizando a se guinte expressão:

$$
u_{r}^{\prime}=\sum_{j=0}^{r}\left(\begin{array}{l}
r \\
j
\end{array}\right) \mu_{r-j} \mu_{1}^{\prime j}=\left(\mu+\mu_{1}^{\prime}\right)^{(r)}
$$

onde, no desenvolvimento da expressão $\left(\mu+\mu_{1}^{\prime}\right)^{\mathrm{r}}$ para a primeira par cela $\circ \underline{r}$ funciona como indice, e para a segunda parcela funciona co mo expoente. 
18.

Demonstração:

Suponha-se $\underline{a}$ e $\underline{b}$ duas variáveis, tais que $b-a=c$, e denote-se por $\mu^{\prime}(a)$ e $\mu^{\prime}(b)$ os momentos em relação a a e $\underline{b}$, respectivamente. Pelo teorema binomial, tem-se a seguinte igualdade:

$$
\begin{aligned}
& (x-a)^{r}=(x-b+b-a)^{r}=(x-b+c)^{r}=[(x-b)+c]^{r} \\
& (x-a)^{r}=\sum_{j=0}^{r}\left(_{j}^{r}\right)(x-b)^{r-j} c^{j} \\
& \text { Sendo } \mu_{r}^{\prime}(a)=\int_{-\infty}^{+\infty}(x-a)^{r} d P, \quad c o m \quad d P=f_{X}(x) d x, \\
& \mu_{r}^{\prime}(a)=\int_{-\infty}^{+\infty} \sum_{j=0}^{r}{ }_{j}^{r}(x-b)^{r-j} c^{j} d P \\
& \mu_{r}^{\prime}(a)=\sum_{j=0}^{r}{ }_{j}^{r} c_{j}^{j} \int_{-\infty}^{+\infty}(x-b)^{r-j} d P
\end{aligned}
$$

e

$$
\mu_{(r-j)}^{\prime}(b)=\int_{-\infty}^{+\infty}(x-b)^{r-j} d P
$$

tem-se

$$
\mu_{r}^{\prime}(a)=\sum_{j=0}^{r}\left({ }_{j}^{r}\right) c^{j} \mu_{(r-j)}^{\prime}(b)
$$

ou, simbolicamente escrevendo,

$$
\mu_{r}^{\prime}(a)=\left[\mu^{\prime}(b)+c\right]^{(r)}
$$

onde, convencionalmente, na expressão, $\left\{\mu^{\prime}(b)\right\}^{j}$ representa $\mu_{j}^{\prime}(b)$.

Se $a=\mu_{i}^{\prime}$ e b é uma origem arbiträria,

$$
\mu_{r}=\sum_{j=0}^{r}\left(\begin{array}{l}
r \\
j
\end{array}\right) c^{j} \mu_{(r-j)}^{\prime}(b)=\left[\mu_{(b)}^{\prime}+c\right]^{(r)}
$$


19.

Se $b=\mu_{1}^{\prime}$ e $\underline{a}$ é uma origem arbiträria,

$\left.\mu_{r}^{\prime}(a)=\sum_{j=0}^{r}\left(\begin{array}{l}r \\ j\end{array}\right) c^{j} \mu_{(r-j}\right)=[\mu+c]^{(r)}$

Se $b=0$ e $a=\mu_{i}^{\prime}$

$\mu_{r}=\sum_{j=0}^{r}\left(\begin{array}{l}r \\ j\end{array}\right) \mu_{(r-j)}^{\prime}\left(-\mu_{i}^{\prime}\right)^{j}=\left[\mu^{\prime}-\mu_{1}^{\prime}\right]^{(r)}$

Se $a=0$ e $b=\mu_{1}^{\prime}$

$\mu_{r}^{\prime}=\sum_{j=0}^{r}\left(\begin{array}{l}r \\ j\end{array}\right) \mu_{(r-j)} \mu_{1}^{\prime} j=\left[\mu+\mu_{i}\right]^{(r)}$

3.2.5.1 - Câlculo de alguns momentos pela relação geral entre momentos centrados e momentos em relação à origem

Como $\mu_{r}^{\prime}=\sum_{j=0}^{r}\left({ }_{j}^{r}\right) \mu_{r-j} \mu_{1}^{\prime j}=\left(\mu+\mu_{1}^{\prime}\right)^{r}$, se $r=2$, tem-se:

$$
\begin{aligned}
\mu_{2}^{\prime} & =\sum_{j=0}^{2}\left(\begin{array}{l}
2 \\
j
\end{array}\right) \mu_{2-j} \mu_{1}^{j} \\
& =\left(\begin{array}{l}
2 \\
0
\end{array}\right) \mu_{2} \mu_{1}^{\prime 0}+\left(\begin{array}{l}
2 \\
1
\end{array}\right) \mu_{1} \mu_{1}^{\prime 2}+\left(\begin{array}{l}
2 \\
2
\end{array}\right) \mu_{0} \mu_{1}^{\prime 2}
\end{aligned}
$$

$$
\begin{array}{rl}
\text { como } \mu_{1}=0 \text { e } \mu_{0} & E\left(X-m_{X}\right)^{0}=1, \\
\mu_{2}^{\prime} & =\mu_{2}+2.0 \cdot \mu_{1}^{\prime}+1.1 \cdot \mu_{1}^{\prime 2} \\
\mu_{2}^{\prime} & =\mu_{2}+\mu_{1}^{\prime 2} \text { e } \mu_{2}=\mu_{2}^{\prime}-\mu_{1}^{\prime 2} .
\end{array}
$$

se $r=3$, tem-se:

$$
\mu_{3}^{\prime}=\sum_{j=0}^{3}\left(\begin{array}{l}
3 \\
j
\end{array}\right) \mu_{3-j} \mu_{i}^{\prime j}
$$




$$
\begin{aligned}
& \mu_{3}^{\prime}=\left(\begin{array}{l}
3 \\
0
\end{array}\right) \mu_{3} \mu_{1}^{\prime 0}+\left(\begin{array}{l}
3 \\
1
\end{array}\right) \mu_{2} \mu_{1}^{\prime}+\left(\begin{array}{l}
3 \\
2
\end{array}\right) \mu_{1} \mu_{1}^{\prime 2}+\left(\begin{array}{l}
3 \\
3
\end{array}\right) \mu_{0} \mu_{1}^{\prime 3} \\
& \mu_{3}^{\prime}=\mu_{3}+3 \mu_{2}^{\prime} \mu_{1}^{\prime}+\mu_{3}^{3} \text { e } \mu_{3}=\mu_{3}^{\prime}-3 \mu_{1}^{\prime} \mu_{2}^{\prime}+2 \mu_{1}^{\prime 3}
\end{aligned}
$$

se $r=4$, tem-se:

$$
\begin{aligned}
& \mu_{4}^{\prime}=\sum_{j=0}^{4}\left(\begin{array}{l}
4 \\
j
\end{array}\right) \mu_{4-j} \mu_{1}^{\prime j} \\
& \mu_{4}^{\prime}=\mu_{4}+4 \mu_{3} \mu_{1}^{\prime}+6 \mu_{2} \mu_{1}^{\prime 2}+\mu_{1}^{\prime 4} \\
& \mu_{4}=\mu_{4}^{\prime}-4 \mu_{1}^{\prime} \mu_{3}^{\prime}+6 \mu_{1}^{2} \mu_{2}^{\prime}-3 \mu_{1}^{\prime 4}
\end{aligned}
$$

\subsection{6 - Momentos absolutos}

Define-se momento absoluto de ordem $\underline{r}$ em relação a a e nota-se por $v_{r}^{\prime} a$

$$
v_{r}^{\prime}=E\left[|X-a|^{r}\right] \quad, \quad r=0,1,2, \ldots
$$

ou seja,

$$
v_{r}^{\prime}=\sum_{j=1}^{n}\left|x_{j}-a\right|^{r} f_{x}\left(x_{j}\right)
$$

se $x$ é V.A. discreta, e

$$
v_{r}^{\prime}=\int_{-\infty}^{+\infty}|x-a|^{r} d P
$$

se $x$ é V.A. continua.

$$
\text { Se } a=m_{X} \text {, tem-se o momento absoluto de ordem } \underline{r} \text { em }
$$
relação à média, e nota-se por $v_{r}$ 


$$
v_{r}=E\left[\left|x-m_{X}\right|^{r}\right]=\left\{\begin{array}{cc}
\sum_{j=1}^{n}\left|x_{j}-m_{X}\right|^{r} f_{X}\left(x_{j}\right), & \text { se } x \text { e v.A. dis- } \\
\text { creta } & \text { se } x \text { é V.A. contI- } \\
\int_{-\infty}^{+\infty}\left|x-m_{X}\right|^{r} d P, & \text { nua }
\end{array}\right.
$$

Se existe o momento de ordem $\underline{r}$, o momento absoluto existe para qualquer ordem inferior a $\underline{r}$. Se $\underline{r} \bar{e}$ par, o momento abso luto é igual ao momento comum, e se o dominnio da distribuição é positivo, os momentos absolutos, simétricos em relação à qualquer pon to, são iguais aos correspondentes momentos comuns.

\subsection{7 - Momentos fatoriais}

Define-se momento fatorial de ordem $r(r>0)$, em re lação a uma origem arbitrāria, e indica-se por $\mu^{\prime}[\mathrm{r}]$, a

$$
\begin{aligned}
& \mu_{[r]}^{\prime}=\sum_{j=-\infty}^{\infty}\left(x_{j}-a\right)^{[r]} f_{X}\left(x_{j}\right), \text { se } x \text { é V.A. discreta } \\
& \mu_{[r]}^{\prime}=\int_{-\infty}^{+\infty}(x-a)^{[r]} f_{X}(x) d x, \text { se } x \text { é V.A. contínua }
\end{aligned}
$$

onde $\left(x_{j}-a\right)[r]=(x-a)(x-a-1)(x-a-2) \ldots(x-a-r+1) ;$ e, se $a=0$, tem-se o momento fatorial de ordem $r$ em relação à origem.

$$
\begin{aligned}
& \sum_{\mathrm{x}} \mathrm{x}(\mathrm{x}-1) \ldots(\mathrm{x}-\mathrm{r}+1) \mathrm{f}_{\mathrm{X}}(\mathrm{x}) \text {, } \\
& \text { se } x \text { ë V.A. discreta } \\
& \mu^{\prime}[\mathrm{r}]=E[X(X-1) \ldots(X-r+1)]=\left\{\begin{array}{c}
\int_{-\infty}^{+\infty} x(x-1) \ldots(x-r+1) f_{X}(x) d x, \\
\text { se } x \text { é V.A. continua }
\end{array}\right.
\end{aligned}
$$


Os momentos fatoriais são usados quase que unicamente para distribuições discretas e, por isso, a maior parte da literatura os definem apenas com a notação de somatōrio.

Esses momentos não apresentam grande importância em teoria estatística, porém, são citados no presente trabalho, porque fornecem förmulas bastante concisas para momentos de certas distribuições discretas do tipo binomial.

0 momento fatorial de ordem $\underline{r}$, em relação à média, ẻ notado por $\mu[r]$ e definido por

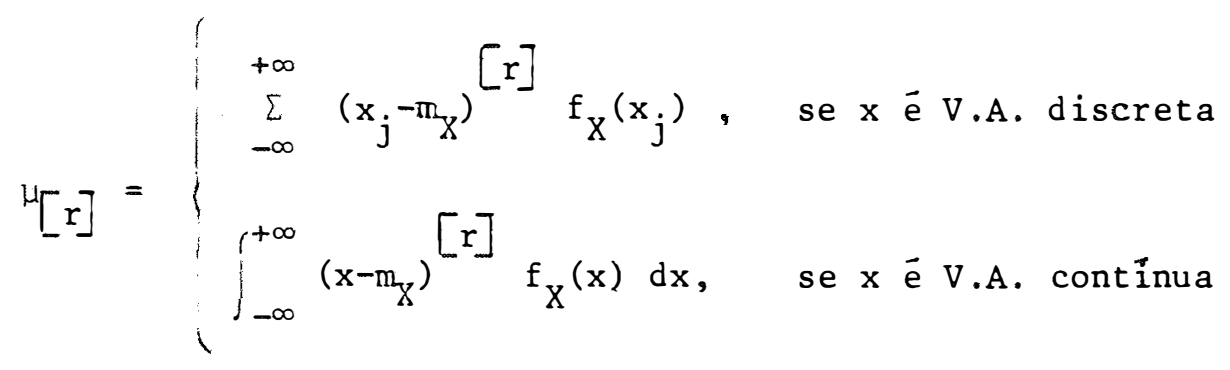

Segundo KENDALl e STUART (1976, p. 66), os momentos fatoriais obedecem leis de transformação similares às dos momentos comuns, de forma que

$$
\mu_{[r]}^{\prime}=\sum_{j=v}^{r}\left({ }_{j}^{r}\right) \mu[r-j] \mu_{[1]}^{j}=\left(\mu+\mu^{\prime}[1]\right)^{[r]}
$$

ë a relação de correspondência entre os momentos fatoriais em relação à origem à média.

Ainda segundo KENDALL e STUART (1976) tem-se a seguinte relação, por expansão direta da definição de momento fato- 
23.

rial, entre momentos fatoriais e momentos comuns

$$
\begin{aligned}
& \left.\mu_{1}^{2}\right]=\mu_{1}^{\prime} \\
& \mu_{[2]}^{1}=\mu_{2}^{\prime}-\mu_{1}^{\prime} \\
& \mu_{[3]}^{\prime}=\mu_{3}^{\prime}-3 \mu_{2}^{\prime}+2 \mu_{1}^{\prime} \\
& \left.\mu_{4}^{1}\right]=\mu_{4}^{\prime}-6 \mu_{3}^{\prime}+11 \mu_{2}^{\prime}-6 \mu_{1}^{\prime}
\end{aligned}
$$

e, por inversão,

$$
\begin{aligned}
& \mu_{2}^{\prime}=\mu_{[2]}^{\prime}+\mu_{[1]}^{\prime} \\
& \mu_{3}^{\prime}=\mu_{[3]}^{1}+3 \mu_{[2]}^{1}+\mu_{[1]}^{2} \\
& \mu_{4}^{\prime}=\mu_{[4]}^{2}+6 \mu^{2}[3]+7 \mu^{2}[2]+\mu^{1}[1]
\end{aligned}
$$

KENDALL e STUART (1976) citam, também, que FRISCH (1926) demonstrou a fórmula geral para os momentos fatoriais que se segue:

$$
\mu_{r}^{\prime}(a)=\sum_{j=0}^{r}\left\{\left(\begin{array}{lll}
r & B_{r-j}^{(-j)} & (c) h^{r-j}
\end{array} \mu^{\prime} j\right] \quad(b)\right\}
$$

e

$$
\mu_{[r]}^{\prime}(a)=\sum_{j=0}^{r}\left\{\left(_{j}^{r}\right) B_{r-j}^{(r+1)}(c+h) h^{r-j} \mu_{j}^{\prime}(b)\right\}
$$

onde $\mathrm{B}_{\mathrm{r}}^{(\mathrm{n})}(\mathrm{x})$ é o polinômio de Bernoulli de ordem $\underline{\mathrm{n}}$ e grau $\underline{\mathrm{r}}$ en $\underline{x}$. 


\section{3 - Funções Geradoras de Momentos}

Calcular momentos pelas definições dadas torna-se um trabalho exaustivo, a partir do instante em que se precisa pesquisar momentos de ordens mais elevadas. Em função disso,procura-se um método que permita obter momentos de modo mais imediato, sem necessariamente aplicarem-se as definições. Para tanto, define-se e cons troi-se a função geradora de momentos, designada por $\mathrm{m}_{X}(t)$, ou simplesmente $m(t)$, se não hā dúvida quanto à variāvel.

\section{3 .1 - Definição}

Define-se como função geradora de momentos de uma variāvel aleatöria $X$ a função

$$
\begin{aligned}
& m_{X}(t)=E\left[e^{t X}\right]=\sum_{x} e^{t x} f_{X}(x), \text { se } x \vec{e} V \cdot A \cdot d i s c r e t a, e \\
& m_{X}(t)=E\left[e^{t X]}=\int_{-\infty}^{+\infty} e^{t x} f_{X}(x), \text { se } x\right. \text { e V.A. continua, }
\end{aligned}
$$

que existe (se a série, ou a integral forem convergentes), para todo $\mathrm{t}$ no intervalo $-\mathrm{h}<\mathrm{t}<\mathrm{h}, \mathrm{h}>0$.

A função geradora de momentos, $m_{X}(t)=E\left[e^{t X}\right] \vec{e}$ tam bém chamada "transformada de Laplace" de $\mathrm{f}_{X}(\mathrm{x})$.

Para $t=0, \mathrm{~m}_{\mathrm{X}}(0)$ sempre existe e è igual a 1 . Entre tanto, ela pode não existir para $t \neq 0$.

Se a f.g.m. existe, então tem derivadas continuas dentro de alguma vizinhança da origem, de forma que, se derivada $\underline{r}$ 
vezes em relação a $\underline{t}$ (considerando-se o caso contínuo como ilustração) tem-se:

$$
m_{x}^{r}(t)=\frac{d^{r}}{d t^{r}} m_{X}(t)=\int_{-\infty}^{+\infty} x^{r} e^{t x} f_{x}(x) d x
$$

e, tomando-se $t \rightarrow 0$, obtém-se

$$
\mu_{(r)}^{\prime}=E\left(X^{r}\right)=m_{X}^{r}(0)=\left.\frac{d^{r}}{d t^{r}} m_{X}(t)\right|_{t=0}=\int_{-\infty}^{+\infty} x^{r} f_{X}(x) d x
$$

o que evidencia que os momentos de uma distribuição podem ser obtidos a partir da f.g.m., por derivação, daí o seu nome.

$$
\text { Para verificar como } m_{X}(t) \text { define momentos, pode-se, }
$$

alternativamente, efetuar a sua expansão em série

$$
\begin{aligned}
& \mathrm{m}_{X}(t)=E\left[e^{t X}\right]=E\left[1+\frac{X t}{1 !}+\frac{X^{2} t^{2}}{2 !}+\frac{X^{3} t^{3}}{3 !}+\frac{X^{4} t^{4}}{4 !}+\cdots\right] \\
& m_{X}(t)=E(1)+E[X t]+E\left[\frac{X^{2} t^{2}}{2 !}\right]+E\left[\frac{X^{3} t^{3}}{3 !}\right]+\cdots \\
& m_{X}(t)=1+t E(X)+\frac{t^{2}}{2 !} E\left(X^{2}\right)+\frac{t^{3}}{3 !} E\left(X^{3}\right)+\cdots
\end{aligned}
$$

ou seja,

$$
\begin{aligned}
& m_{X}(t)=\mu_{0}^{\prime}+t \mu_{1}^{\prime}+\frac{t^{2}}{2 !} \mu_{2}^{\prime}+\frac{t^{3}}{3 !} \mu_{3}^{\prime}+\ldots \\
& m_{X}(t)=\sum_{j=0}^{\infty} \frac{1}{j !} \mu_{j}^{\prime} t^{j},
\end{aligned}
$$


onde fica claro que $m_{X}(t)$ representa uma soma de momentos em relação à origem, e evidencia que $\mu_{r}^{\prime}$ pode ser obtido através de $m_{X}(t)$, pois é o coeficiente de $\frac{t^{r}}{r !}$ na expansão.

Por outro lado, desenvolvendo-se $m_{X}(t)$ pela série de MacLaurin, tem-se

$$
m_{X}(t)=m_{X}(0)+\frac{t}{1 !} m_{X}^{\prime}(0)+\frac{t^{2}}{2 !} m_{X}^{\prime \prime}(0)+\frac{t^{3}}{3 !} m_{X}^{\prime \prime \prime}(0)+\ldots
$$

e, comparando-se (1) e (2), verifica-se que

$$
\begin{aligned}
& \mathrm{m}_{X}(0)=1 \quad ; \quad \mu_{0}^{\prime}=1 \quad ; \text { ond e } m_{X}^{\prime}(0)=\left.\frac{d}{d t}\left[m_{X}(t)\right]\right|_{t=v} \\
& m_{X}^{\prime}(0)=\mu_{1}^{\prime} \\
& \mathrm{m}_{\mathrm{X}}^{\prime \prime}(0)=\mu_{2}^{\prime} \\
& \mathrm{m}_{X}^{\prime \prime \prime}(0)=\mu_{3}^{\prime} \\
& m_{X}^{\prime \prime}(0)=\left.\frac{d^{2}}{d t^{2}}\left[m_{X}(t)\right]\right|_{t=0} \\
& m_{x}^{\prime \prime \prime}(0)=\left.\frac{d^{3}}{d t^{3}}\left[m_{x}(t)\right]\right|_{t=0}
\end{aligned}
$$

o que demonstra, mais uma vez, que $\mu_{r}^{\prime}$ é o coeficiente de $\frac{t^{r}}{r !}$ na expansão de $m_{X}(t)$.

Com isso, demonstra-se que as derivadas sucessivas de $m_{X}(t)$, no ponto zero, fornecem os momentos de ordem desejada. 
27 .

3.3.2 - Algumas propriedades das funções geradoras de momentos

3.3.2.1) $m_{X}(0)=1$,

$$
\begin{aligned}
\text { pois, } \mathrm{m}_{X}(0)=E\left[\mathrm{e}^{0}\right]=E[1]=1 . \\
\\
\quad 3.3 .2 .2) \mathrm{m}_{X+d}(t)=\mathrm{e}^{t d} \mathrm{~m}_{X}(t)
\end{aligned}
$$

onde d è uma constante, pois:

$$
\begin{aligned}
m_{X+d}(t) & =E\left[e^{t(X+d)}\right]=E\left[e^{t X+t d}\right]=E\left[e^{t X} e^{t d}\right] \\
& =E\left[e^{t d}\right] E\left[e^{t X]}=e^{t d} E\left[e^{t x}\right]\right. \\
3.3 .2 .3) m_{c X}(t) & =m_{X}(c t),
\end{aligned}
$$

onde c e uma constante, pois

$$
m_{c X}(t)=E\left[e^{t c X}\right]=E\left[e^{(t c) X}\right]
$$

3.3.2.4) $m_{c X+d}(t)=e^{t d} m_{X}(c t)$,

pois:

$$
\begin{aligned}
m_{c X+d}(t) & =E\left[e^{(c X+d) t}\right]=E\left[e^{(c t) X} e^{d t}\right] \\
& =e^{t d} E\left[e^{(c t) X}\right]
\end{aligned}
$$




$$
3.3 .2 .5) m_{c g(X)}(t)=m_{g}(X)(c t) \text {, }
$$

onde c è uma constante e $g(X)$ é função de uma V.A. x, pois,

$$
\begin{aligned}
& m_{c g(X)}(t)=E\left[e^{c g(X) t}\right]=E\left[e^{(c t) g(X)}\right]=m_{g(X)}(c t) \\
& 3.3 .2 .6) m_{g(X)+c}(t)=e^{c t} m_{g(X)}(t)
\end{aligned}
$$

pois,

$$
\begin{aligned}
m_{g(x)+c}(t) & =E\left\{e^{[g(x)+c] t}\right\}=E\left[e^{g(x) t} e^{c t}\right] \\
& =e^{c t} m_{g(x)} t
\end{aligned}
$$

E necessärio observar-se que essas propriedades estão sujeitas à existência das esperanças.

\subsection{3 - Funções geradoras de momentos conjuntos}

\subsubsection{1 - Definição}

Define-se função geradora de momentos de uma variável aleatória conjunta $\left(x_{1}, \ldots, x_{K}\right)$ ou do vetor aleatório $x=\left(x_{1} \ldots\right.$ $\left.\ldots \mathrm{x}_{\mathrm{K}}\right)^{\prime} \mathrm{a}$

$$
m_{X}\left(t_{1}, \ldots, t_{K}\right)=m_{X_{1}}, \ldots, x_{K}\left(t_{1}, \ldots, t_{K}\right)=E\left[e^{\sum_{j=1}^{K} t_{j} x_{j}}\right]
$$

se a esperança existe para todos os valores de $t_{1}, t_{2}, \ldots, t_{k}$ tal que $-h<t_{j}<h, h>0, j=1,2, \ldots, k$. 
Obtëm-se o momento de ordem $\underline{r}$ de $x_{j}$, a partir da f.g.m. conjuntos, por derivação parcial, como a seguir:

$$
\begin{array}{r}
E\left(x_{j}^{r}\right)=\frac{\partial^{r}}{\partial t_{j}^{r}} m_{x_{1}}, \ldots,\left.x_{K}\left(t_{1}, \ldots, t_{K}\right)\right|_{t_{1}=t_{2}=\ldots=t_{K}=0} \\
E\left(x_{i}^{r} x_{j}^{s}\right)=\frac{\partial^{r+s}}{\partial t_{i}^{r} \partial t_{j}^{s}} m_{X_{1}}, \ldots,\left.x_{K}\left(t_{1}, \ldots, t_{K}\right)\right|_{t_{1}=t_{2}=\ldots=t_{k}=0}
\end{array}
$$

e, genericamente:

$E\left[x_{1}^{n_{1}}, \ldots, x_{K}^{n_{K}}\right]=\frac{\partial^{n_{1}+\ldots t_{K}}}{\partial t_{1}^{n_{1}} \ldots \partial^{n_{K}}} m_{x_{1}}, \ldots,\left.x_{K}\left(t_{1}, \ldots, t_{K}\right)\right|_{t_{1}=t_{2}=\ldots=t_{K}=0}$

\subsubsection{2 - Algumas propriedades das f.g.m. conjuntos}

$\cdot \mathrm{m}_{\mathrm{x}}, \ldots, \mathrm{x}_{\mathrm{K}}(0, \ldots, 0)=1$

$\cdot m_{x_{1}+d_{1}}, \ldots, x_{K}+d_{K}\left(t_{1}, \ldots, t_{K}\right)=e^{t_{1} d_{1}+\ldots+t_{K} d_{K}} m_{x_{1}}, \ldots, x_{K}\left(t_{1}, \ldots, t_{K}\right)$

$\cdot m_{c_{1} x_{1}}, \ldots, c_{K} x_{K}\left(t, \ldots, t_{K}\right)=m_{X_{1}}, \ldots, x_{K}\left(c_{1} t_{1}, \ldots, c_{K} t_{K}\right)$

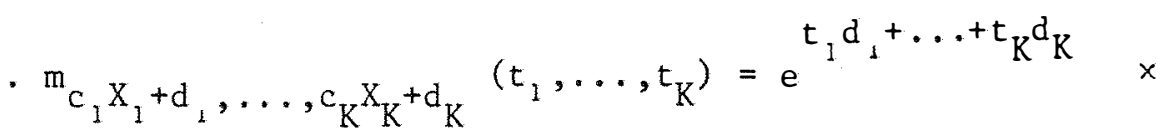

$$
\times m_{x_{1}}, \ldots, x_{K}\left(c_{1} t_{1}, \ldots, c_{K} t_{K}\right)
$$




\subsubsection{3 - Funções geradoras de momentos marginais}

Define-se função geradora de momentos marginal e nota-se por $m_{x_{j}}\left(t_{j}\right)$ a

$$
\begin{gathered}
m_{x_{j}}\left(t_{j}\right)=m_{x_{1}}, \ldots, x_{k} \quad\left(0, \ldots, 0, t_{j}, 0, \ldots, 0\right) \\
j=1,2, \ldots, k
\end{gathered}
$$

A função geradora de momentos marginal de $x_{i}$ e $x_{j}$,por exemplo, è definida por

$$
\begin{aligned}
m_{x_{i}, x_{j}}\left(t_{i}, t_{j}\right)=m_{1}, \ldots, x_{K}\left(0, \ldots, 0, t_{i}, 0, \ldots, 0, t_{j}, 0, \ldots, 0\right) \\
3.3 .3 .4 \text { - } \begin{aligned}
\text { Função geradora de momentos conjuntos e } \\
\text { independência estocãstica mútua de }\left(x_{1}, \ldots\right. \\
\left.\ldots, x_{K}\right)
\end{aligned}
\end{aligned}
$$

- Independência Estocástica:

Sejam as variáveis aleatōrias $x_{1}, x_{2}, \ldots, x_{K}$, tendo a função de densidade de probabilidade conjunta $\mathrm{f}_{\mathrm{X}_{1}}, \ldots, \mathrm{x}_{\mathrm{K}}\left(\mathrm{x}_{1}, \ldots\right.$ $\left.\ldots, x_{K}\right)$, e as funções de densidade de probabilidade marginal $\mathrm{f}_{\mathrm{X}_{1}}\left(\mathrm{x}_{1}\right), \ldots, \mathrm{f}_{\mathrm{X}_{\mathrm{K}}}\left(\mathrm{x}_{\mathrm{K}}\right)$.

As variáveis aleatōrias $x_{1}, \ldots, X_{K}$ são estocasticamente independentes, se e somente se,

$$
f_{X_{1}}, \ldots, x_{K}\left(x_{1}, \ldots, x_{K}\right)=f_{X_{1}}\left(x_{1}\right) f_{X_{2}}\left(x_{2}\right) \ldots f_{X_{K}}\left(x_{K}\right)
$$


Pode-se demonstrar que uma condição necessäria e suficiente para a independência estocástica mútua de $\left(x_{1}, \ldots, x_{K}\right)$ é que

$$
m_{x_{1}}, \ldots, x_{K}\left(t_{1}, \ldots, t_{K}\right)=\prod_{j=1}^{k} m\left(0, \ldots, 0, t_{j}, 0, \ldots, 0\right)
$$

ou seja, que a f.g.m. para a distribuição conjunta de $\left(x_{1}, x_{2}, \ldots\right.$, $\mathrm{X}_{\mathrm{K}}$ ) seja única, e unicamente determine a distribuição conjunta das $K$ variáveis (e consequentemente todas as distribuições marginais)

Demons tração:

Se $\left(X_{1}, X_{L}, \ldots, x_{K}\right)$ são estocasticamente independen tes,

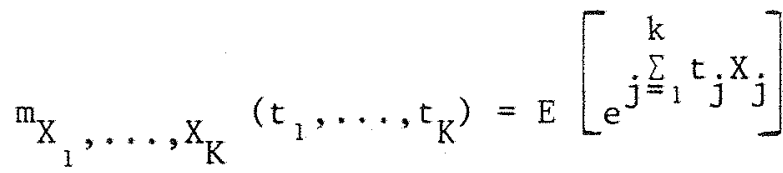

$$
\begin{aligned}
& =E\left[e^{t_{1} x_{1}} e^{t_{L} x_{2}} \ldots e^{t_{K} x_{K}}\right] \\
& =E\left[e^{t_{1} x_{1}}\right] E\left[e^{t_{2} X_{2}}\right] \ldots E\left[e^{t_{K} X_{K}}\right] \\
& =\mathrm{m}_{\mathrm{x}}, \ldots, \mathrm{x}_{\mathrm{K}}\left(t_{1}, 0, \ldots, 0\right) \quad x \\
& x \mathrm{~m}_{2}, \ldots, \mathrm{x}_{\mathrm{K}}\left(0, \mathrm{t}_{2}, 0, \ldots, 0\right) \ldots \\
& \ldots \mathrm{m}_{\mathrm{x}_{1}}, \ldots, \mathrm{x}_{\mathrm{K}}\left(0,0, \ldots, 0, \mathrm{t}_{\mathrm{K}}\right)
\end{aligned}
$$


ou seja, a independência estocästica de $\left(x_{1}, \ldots, x_{K}\right)$ implica que a f.g.m. da distribuição conjunta esteja para o produto dos fatores das f.g.m. de suas distribuições marginais.

Supondo-se, a seguir, que a f.g.m. para a distribuiçao conjunta de $\left(x_{1}, \ldots, X_{K}\right)$ é dada por

$$
\prod_{j=1}^{k} m_{1}, \ldots, x_{K}\left(0, \ldots, 0, t_{j}, 0, \ldots, 0\right)
$$

com $x_{j}$ continua tal que

$$
m_{x_{j}}\left(t_{j}\right)=m_{x_{1}}, \ldots, x_{k}\left(0, \ldots, 0, t_{j}, \ldots, 0\right)=\int_{-\infty}^{+\infty} e^{t_{j} x_{j}} f_{x_{j}}\left(x_{j}\right) d x_{j}
$$

tem-se que

$$
\begin{aligned}
& \mathrm{II}_{j=1}^{k} \mathrm{~m}_{\mathrm{X}_{1}}, \ldots, \mathrm{X}_{\mathrm{K}}\left(0, \ldots, 0, t_{j}, 0, \ldots, 0\right)=\left[\int_{-\infty}^{+\infty} e^{t_{1} \mathrm{X}_{1}} \mathrm{E}_{\mathrm{X}_{1}}\left(\mathrm{x}_{1}\right) \mathrm{dx} \mathrm{x}_{1}\right] \ldots \\
& \cdots\left[\int_{-\infty}^{+\infty} e^{t j^{x} j} f_{x_{j}}\left(x_{j}\right) d x j\right] \cdots \\
& \cdots\left[\int_{-\infty}^{+\infty} e^{t} \mathrm{~K}^{\mathrm{X}_{\mathrm{K}}} \mathrm{f}_{\mathrm{X}_{\mathrm{K}}}\left(\mathrm{x}_{\mathrm{K}}\right) \mathrm{dx} \mathrm{x}_{\mathrm{K}}\right] \\
& =\int_{-\infty}^{+\infty} \cdots \int_{-\infty}^{+\infty} e^{t_{1} x_{1}+\ldots+t_{K} x_{K}} f_{X_{1}}\left(x_{1}\right) \ldots f_{X_{K}}\left(x_{K}\right) d x_{1} \ldots d x_{K}
\end{aligned}
$$


Como

$$
m_{x_{1}}, \ldots, x_{K}\left(t_{1}, \ldots, t_{K}\right)=\prod_{j=1}^{k} m_{x_{1}}, \ldots, x_{K}\left(0, \ldots, 0, t_{j}, 0, \ldots, 0\right)
$$

tem-se que

$$
\begin{gathered}
m_{x_{1}}, \ldots, x_{K}\left(t_{1}, \ldots, t_{K}\right)=\int_{-\infty}^{+\infty} \ldots \int_{-\infty}^{+\infty} e^{j=1} t_{j}^{k} x_{j} \prod_{j=1}^{k} f_{x_{j}}\left(x_{j}\right) d x_{1}, \ldots, d x_{k} \\
\quad \text { Mas } m_{x_{1}}, \ldots, x_{K}\left(t_{1}, \ldots, t_{K}\right) \text { é a f.g.m.de }\left(x_{1}, \ldots, x_{K}\right),
\end{gathered}
$$

então tambēm o é

$$
m_{x_{1}}, \ldots, x_{k}\left(t_{1}, \ldots, t_{k}\right)=\int_{-\infty}^{+\infty} \ldots \int_{-\infty}^{+\infty} e^{\sum_{j=1}^{k} t_{j} x_{j}} f_{x_{1}}, \ldots, x_{k}\left(x_{1}, \ldots, x_{k}\right) d x_{1}, \ldots, d x_{k}
$$

pois a unicidade da função geradora de momentos implica que as $\underline{k}$ dis tribuições de probabilidade, $\sum_{j=1}^{k} f_{x_{j}}\left(x_{j}\right), e f_{x_{1}}, \ldots, x_{K}\left(x_{1}, \ldots, x_{K}\right)$ sejam iguais, isto é, sf.

$$
m_{x_{i}}, \ldots, x_{k}\left(t_{1}, \ldots, t_{k}\right)=\prod_{j=1}^{k} m_{x_{1}}, \ldots, x_{k}\left(0, \ldots, 0, t_{j}, 0, \ldots, 0\right)
$$

então $\left(X_{1}, \ldots, X_{K}\right)$ são estocasticamente independentes.

Embora se apresente demonstração para variäveis contỉnuas, com variäveis discretas a prova é semelhante, usando-se somatōrios. 


\subsection{4 - Funções geradoras de momentos fatoriais}

\subsubsection{1 - Definição}

Define-se função geradora de momentos fatoriais, e in dica-se por $\eta_{X}(t)$ a

$$
\eta_{X}(t)=E[t] \quad, \quad t \in \mathbf{R},
$$

se essa esperança existe.

Esta função ē tambēm conhecida como a MELLIN ou MELLIN-STIELTJES transformada de $\mathrm{f}(\mathrm{x})$.

$\vec{E}$ utilizada para gerar momentos fatoriais de modo aná logo aos momentos comuns obtidos através de $E\left(e^{t x}\right)$, exceto que $t$ deve ser substituído por 1 , e não por zero. Ou seja, como

$$
\begin{aligned}
\frac{d^{r}}{d t^{r}} \quad \eta_{X}(t) & =\frac{d^{r}}{d t^{r}} E\left[t^{x}\right] \\
& =E\left[\frac{d^{r}}{d t^{r}} t^{x}\right]=E\left[X(X-1) \ldots(X-r+1) t^{X-r}\right]
\end{aligned}
$$

tem-se que

$$
\mu^{\prime}[r]=\left.\frac{d^{r}}{d t^{r}} \eta_{X}(t)\right|_{t=1}=E[X(X-1) \ldots(x-r+1)]
$$

Apresenta-se essa f.g.m. fatorial nesse trabalho,pois a mesma pode, algumas vezes, simplificar a obtenção de momentos para algumas distribuições discretas. 


$$
\text { Observa-se também que } \eta_{X}(t)=m_{X}(\ell n t) \text {, para } t>0 \text {. }
$$

Nota (1)

Sendo

$$
\sigma^{2}(X)=E\left(X^{2}\right)-[E(X)]^{2}
$$

e

$$
E\left(X^{2}\right)=E[X(X-1)]+E(X),
$$

tem-se que

$$
\sigma^{2}(X)=E[X(X-1)]+E(X)-[E(X)]^{2},
$$

o que corresponde a uma expressão da variância de $\mathrm{X}$ em termos da de rivada da f.g.m. fatorial de 2 . ordem.

3.4 - Cálculo da Função Geradora de Momentos, e Principais Momen tos, para Algumas Distribuições Especiais de Probabilidade

Como se propõe este trabalho, passa-se agora à dedução de funções geradoras de momentos das distribuições de probabili dade mais usuais, que são separadas em discretas e contínuas. Não sáo calculados os momentos de ordem elevada, pois a seguir apresenta-se um método mais rápido e eficaz para esses cálculos, que se constitui na utilização da fórmula recorrente de momentos (NOGUEIRA, 1965). Apenas a título de ilustração, são obtidas também algumas fun ções geradoras de momentos fatoriais. 


\subsection{1 - Distribuições discretas}

\subsubsection{1 - Distribuição uniforme discreta}

\subsubsection{1 - Definição}

Define-se uma V.A. discreta X, tendo distribuição uni forme, se a sua densidade é dada por

$$
\begin{aligned}
f_{X}(x)=f_{X}(x ; N)=\left\{\begin{aligned}
\frac{1}{N} & \text { para } x=1,2, \ldots, N \\
0 & \text { para outros valores }
\end{aligned}\right. \\
3.4 .1 .1 .2 \text { - Função geradora de momentos } \\
m_{X}(t)=\underset{j=1}{N} e^{j t} \frac{1}{N},
\end{aligned}
$$

pois,

$$
\begin{aligned}
& m_{X}(t)=E\left[e^{t X}\right]=E\left[e^{t X} \frac{1}{N}\right]=\sum_{j=1}^{N} e^{j t} \frac{1}{N} \\
& m_{X}(t)=\sum_{j=1}^{N} e^{j t} \frac{1}{N}
\end{aligned}
$$

3.4.1.1.3 - Cálculo dos principais momen tos pela f.g.m.

$$
\mu_{1}^{\prime}=E(X)=\frac{N+1}{2}
$$

pois, 


$$
\begin{aligned}
& m_{X}^{\prime}(t)=\sum_{j=1}^{N} e^{j t} j \frac{1}{N} \\
& m_{X}^{\prime}(0)=\sum_{j=1}^{N} j \frac{1}{N}=\frac{1}{N} \sum_{j=1}^{N} j=\frac{1}{N} \frac{N(N+1)}{2} \\
& m_{X}^{\prime}(0)=\frac{N+1}{2}=\mu_{1}^{\prime} \\
& \mu_{2}^{\prime}=E\left(X^{2}\right)=\frac{(N+1)(2 N+1)}{6 N}
\end{aligned}
$$

pois,

$$
\begin{aligned}
m_{X}^{\prime \prime}(t) & =\sum_{j=1}^{N} e^{j t} j^{2} \frac{1}{N} \\
m_{X}^{\prime \prime}(0) & =\sum_{j=1}^{N} j^{2} \frac{1}{N}=\frac{1}{N} \sum_{j=1}^{N} j^{2}=\frac{1}{N} \frac{(N+1)(2 N+1)}{6} N \\
m_{X}^{\prime \prime}(0) & =\frac{(N+1)(2 N+1)}{6}=\mu_{2}^{\prime} \\
H_{2} & =\operatorname{Var}(X)=\frac{N^{2}-1}{12}
\end{aligned}
$$

pois:

$$
\begin{aligned}
& \mu_{2}=\mu_{2}^{\prime}-\mu_{1}^{2} \\
& \mu_{2}=\frac{(N+1)(2 N+1)}{6}-\frac{(N+1)^{2}}{4}=\frac{N^{2}-1}{12}
\end{aligned}
$$




\subsubsection{2 - Distribuição de Bernoulli}

\subsubsection{1 - Definição}

Define-se uma V.A. discreta X, tendo distribuição de Bernoulli, se a sua densidade é dada por

$$
f_{X}(x)=f_{X}(x ; p)= \begin{cases}p^{x}(1-p)^{1-x} & \text { para } x=0 \text { ou } x=1 \\ 0 & \text { para outros valores }\end{cases}
$$

$\operatorname{com} 0 \leq p \leq 1$ e $q=1-p$

3.4.1.2.2 - Função geradora de momentos

$$
m_{X}(t)=q+p e^{t},
$$

pois,

$$
\begin{aligned}
& m_{X}(t)=E\left[e^{t X}\right]=\Sigma\left[e^{x}(1-p)^{1-x}\right] \\
&=\Sigma\left[e^{t X} p^{X} q^{1-x}\right]=q+e^{t} p \\
& m_{X}(t)=q+e^{t} p \\
& 3.4 .1 .2 .3-\text { Cálculo dos principais momen } \\
& \text { pois, tos pela f.g.m. } \\
& m_{X}^{\prime}(t)=p e^{t} \\
& m_{X}^{\prime}(0)=p
\end{aligned}
$$




$$
\mu_{2}^{\prime}=\mathrm{E}\left(\mathrm{X}^{2}\right)=\mathrm{p}
$$

$$
\text { pois, } \quad \begin{aligned}
\mathrm{m}_{\mathrm{X}}^{\prime \prime}(\mathrm{t}) & =\mathrm{pe} \mathrm{t}^{\mathrm{t}} \\
\mathrm{m}_{\mathrm{X}}^{\prime \prime}(0) & =\mathrm{p}
\end{aligned}
$$$$
\mu_{2}=\operatorname{Var}(X)=p q
$$

pois,

$$
\begin{aligned}
& \mu_{2}=\mu_{2}^{\prime}-\mu_{1}^{2} \\
& \mu_{2}=p-p^{2}=p(1-p)=p q
\end{aligned}
$$

\subsubsection{3 - Distribuição binomial}

\subsubsection{1 - Definição}

Define-se uma V.A. discreta X, tendo distribuição bi nomial de probabilidade, se a sua densidade é dada por:

$$
f_{X}(x)=f_{X}(x ; N, p)= \begin{cases}\left(\begin{array}{l}
N \\
X
\end{array}\right) p^{X} q^{N-x} & \text { para } x=0,1,2, \ldots, N \\
0 & \text { para outros valores }\end{cases}
$$

$\operatorname{com} 0 \leq \mathrm{p} \leq 1$ e $\mathrm{q}=1-\mathrm{p}$.

3.4.1.3.2 - Funçáo geradora de momentos

$$
m_{X}(t)=\left(q+p e^{t}\right)^{N}
$$

pois,

$$
\begin{aligned}
& m_{X}(t)=E\left[e^{t X}\right]=\sum\left[e^{t x}\left(\begin{array}{l}
N \\
x
\end{array}\right) p^{x} q^{N-x}\right] \\
& m_{X}(t)=\sum_{x=0}^{N} e^{t x}\left(\begin{array}{l}
N \\
x
\end{array}\right) p^{x} q^{N-x}=\sum_{x=0}^{N}\left(\begin{array}{l}
N \\
x
\end{array}\right)\left(p e^{t}\right)^{x} q^{N-x}
\end{aligned}
$$




$$
\begin{aligned}
& \mathrm{m}_{X}(t)=\left(\begin{array}{l}
N \\
0
\end{array}\right)\left(p e^{t}\right)^{0} q^{N}+\left(\begin{array}{l}
N \\
1
\end{array}\right)\left(p e^{t}\right)^{1} q^{N-1}+\ldots+\left(\begin{array}{l}
N \\
N
\end{array}\right)\left(p e^{t}\right)^{N} q^{0} \\
& m_{X}(t)=\left(q+p e^{t}\right)^{N}
\end{aligned}
$$

3.4.1.3.3 - Càlculo dos principais momentos pela f.g.m.

$$
\mu_{1}^{\prime}=E(X)=N p
$$

pois,

$$
\begin{aligned}
& m_{X}^{\prime}(t)=N\left(q+p e^{t}\right)^{N-i} p e^{t} \\
& m_{X}^{\prime}(0)=N(q+p)^{N-1} p \\
& m_{X}^{\prime}(0)=N p \\
& \mu_{2}^{\prime}=E\left(X^{2}\right)=N p+N^{2} p^{2}-N p^{2}
\end{aligned}
$$

pois,

$$
\begin{gathered}
m_{X}^{\prime \prime}(t)=N p e^{t}\left(q+p e^{t}\right)^{N-1}+N(N-1)\left(p e^{t}\right)^{2}\left(q+p e^{t}\right)^{N-2} \\
m_{X}^{\prime \prime}(0)=N p(q+p)^{N-1}+N(N-1)\left(p^{2}\right)(q+p)^{N-2} \\
m_{X}^{\prime \prime}(0)=N p+\left(N^{2}-N\right) p^{2}=N p+N^{2} p^{2}-N p^{2} \\
\mu_{2}=\operatorname{Var}(X)=N p q
\end{gathered}
$$

pois,

$$
\begin{aligned}
& \mu_{2}=\mu_{2}^{\prime}-\mu_{1}^{2} \\
& \mu_{2}=N p+N^{2} p^{2}-N p^{2}-N^{2} p^{2} \\
& \mu_{2}=N p(1-p)=N p q
\end{aligned}
$$


Por derivação sucessiva, obtem-se, de forma anäloga,

$$
\begin{aligned}
\mu_{3}^{\prime}= & N p+3 N^{2} p^{2}-3 N p^{2}+N^{3} p^{3}-3 N^{2} p^{3}+2 N p^{3} \\
\mu_{3}= & N p q(q-p) \\
\mu_{4}^{\prime}= & N p+7 N^{2} p^{2}+6 N^{3} p^{3}+N^{4} p^{4}-6 N^{3} p^{4}-18 N^{2} p^{3}+11 N^{2} p^{4}- \\
& \quad-7 N p^{2}-6 N p^{4}+12 N p^{3} \\
\mu_{4}= & 3 N^{2} p^{2} q^{2}+N p q(1-6 p q)
\end{aligned}
$$

3.4.1.3.4 - Função geradora de momentos fatorial

$$
\eta_{X}(t)=(q+p t)^{N}
$$

pois,

$$
\begin{aligned}
& \eta_{X}(t)=E\left[t^{X}\right]=\sum\left[t^{x}\left(\begin{array}{l}
N \\
x
\end{array}\right) p^{x} q^{N-x}\right] \\
& \eta_{X}(t)=\sum_{x=0}^{N} t^{X}\left(\begin{array}{l}
N \\
x
\end{array}\right) p^{x} q^{N-x}=\sum_{x=0}^{N}\left(\begin{array}{l}
N \\
X
\end{array}\right)(t p)^{x} q^{N-x} \\
& \eta_{X}(t)=(q+p t)^{N}
\end{aligned}
$$

$$
\begin{gathered}
3.4 .1 .3 .5- \\
\text { Cálculo dos principais momen } \\
\text { tos pela f.g.m. fatorial } \\
\mu_{1}^{\prime}=E(X)=N p,
\end{gathered}
$$

pois,

$$
\begin{aligned}
& \mu_{[1]}^{\prime}=\left.\frac{d}{d t} n_{X}(t)\right|_{t=1} \\
& \mu_{[1]}^{\prime}=\left.N(q+p t)^{N-1} p\right|_{t=1}
\end{aligned}
$$




$$
\begin{gathered}
\mu_{[1]}^{\prime}=N(q+p)^{N-1} p=N p \quad, \quad \text { e como } \mu_{[1]}^{\prime}=\mu_{1}^{\prime}=N p, \\
\mu_{1}^{\prime}=N p \\
\mu_{2}=\operatorname{Var}(X)=N p q
\end{gathered}
$$

pois,

$$
\left.\frac{d^{2}}{d t^{2}} n_{X}(t)\right|_{t=1}=\left.N(N-1)(q+p t)^{N-2} p^{2}\right|_{t=1}=N(N-1) p^{2}
$$

e como $\operatorname{Var}(X)=E[X(X-1)]+E(X)-[E(X)]^{2}$,

$$
\begin{aligned}
& \mu_{2}=\operatorname{Var}(X)=N^{2} p^{2}-N p^{2}+N p-N^{2} p^{2} \\
& \mu_{2}=\operatorname{Var}(X)=N p(1-p)=N p q
\end{aligned}
$$

\subsubsection{4 - Distribuição de Poisson}

\subsubsection{1 - Definição}

Define-se uma V.A. discreta $X$, tendo distribuição de Poisson, se a sua densidade è dada por:

$$
f_{X}(x)=f_{X}(x ; \lambda)=\left\{\begin{array}{cl}
\frac{e^{-\lambda} \lambda^{x}}{x !} & \text { para } x=0,1,2, \ldots \\
0 & \text { para outros valores }
\end{array}\right.
$$

com o parâmetro $\lambda>0$.

3.4.1.4.2 - Função geradora de momentos

$$
m_{X}(t)=e^{\lambda\left(e^{t}-1\right)}
$$


43.

pois,

$$
\begin{aligned}
& \mathrm{m}_{X}(t)=E\left[e^{t X}\right]=\sum\left[e^{t x} \frac{e^{-\lambda} \lambda^{x}}{x !}\right] \\
& m_{X}(t)=\sum_{x=0}^{\infty} \frac{e^{t x} e^{-\lambda} \lambda^{x}}{x !}=e^{-\lambda} \sum_{x=0}^{\infty} \frac{e^{t x} \lambda^{x}}{x !} \\
& m_{X}(t)=e^{-\lambda} \sum_{x=0}^{\infty} \frac{\left(\lambda e^{t}\right)}{x !} \\
& m_{X}(t)=e^{-\lambda} e^{\lambda e^{t}} \\
& \text { pois, } \sum_{x=0}^{\infty} \frac{\left(\lambda e^{t}\right)}{x !}=e^{\lambda e^{t}} \\
& m_{x}(t)=e^{\lambda\left(e^{t}-1\right)}
\end{aligned}
$$

3.4.1.4.3 - Càlculo dos principais momentos pela f.g.m.

$$
\mu_{1}^{\prime}=E(X)=\lambda
$$

pois,

$$
\begin{aligned}
& m_{X}^{\prime}(t)=\lambda e^{t} e^{\lambda\left(e^{t}-1\right)} \\
& m_{X}^{\prime}(0)=e^{0} \lambda e^{0}=\lambda
\end{aligned}
$$$$
\mu_{2}^{\prime}=E\left(X^{2}\right)=\lambda^{2}+\lambda
$$

pois,

$$
\begin{aligned}
& m_{X}^{\prime \prime}(t)=\lambda e^{\lambda e^{t}-\lambda+t} \quad\left(\lambda e^{t}+1\right) \\
& m_{X}^{\prime \prime}(0)=\lambda e^{\lambda-\lambda}(\lambda+1)=\lambda^{2}+\lambda
\end{aligned}
$$




$$
\mu_{2}=\operatorname{Var}(\mathrm{X})=\lambda
$$

pois,

$$
\begin{aligned}
& \mu_{2}=\mu_{2}^{\prime}-\mu_{1}^{2} \\
& \mu_{2}=\lambda^{2}+\lambda-\lambda^{2}=\lambda
\end{aligned}
$$

Por derivação sucessiva obtém-se, de modo análogo,

$$
\begin{aligned}
& \mu_{3}^{\prime}=\lambda^{3}+3 \lambda^{2}+\lambda \\
& \mu_{3}=\lambda \\
& \mu_{4}^{\prime}=\lambda^{4}+6 \lambda^{3}+7 \lambda^{2}+\lambda \\
& \mu_{4}=\lambda+3 \lambda^{2}
\end{aligned}
$$

$$
\begin{gathered}
\text { 3.4.1.4.4 - } \begin{aligned}
& \text { Funçāo geradora de momentos } \\
& \text { fatorial } \\
\eta_{X}(t) & =e^{\lambda(t-1)}
\end{aligned}
\end{gathered}
$$

pois,

$$
\begin{aligned}
& \eta_{X}(t)=E[t X]=\sum\left[t^{x} \frac{e^{-\lambda} \lambda^{x}}{x !}\right] \\
& \eta_{X}(t)=e^{-\lambda \sum_{x=v}^{\infty} \frac{(\lambda t)^{x}}{x !}=e^{-\lambda} e^{\lambda t}} \\
& \eta_{X}(t)=e^{\lambda(t-1)}
\end{aligned}
$$

3.4.1.4.5 - Cálculo dos principais momen tos pela f.g.m. fatorial

$$
\mu_{1}^{\prime}=\mu_{[1]}^{\prime}=E(X)=\lambda
$$


pois,

$$
\begin{gathered}
\left.\mu F_{1}\right]=\left.\frac{d}{d t} \eta_{X}(t)\right|_{t=1} \\
\mu_{[1]}^{\prime}=\left.\frac{d}{d t} e^{\lambda(t-1)}\right|_{t=1}=\left.e^{\lambda(t-1)} \lambda\right|_{t=1}=e^{0} \lambda=\lambda \\
\mu_{2}=\operatorname{Var}(X)=\lambda
\end{gathered}
$$

pois,

$$
\left.\frac{d^{2}}{d t^{2}} \eta_{X}(t)\right|_{t=1}=\left.\lambda^{2} e^{\lambda(t-1)}\right|_{t=1}=\lambda^{2}
$$

e

$$
\begin{aligned}
& \operatorname{Var}(X)=E[X(X-1)]+E(X)-[E(X)]^{2} \\
& \operatorname{Var}(X)=\lambda^{2}+\lambda-\lambda^{2}=\lambda
\end{aligned}
$$

3.4.1.5 - Distribuição geométrica (ou Pascal)

$$
3.4 .7 .5 .1 \text { - Definição }
$$

Define-se uma V.A. discreta X, tendo distribuição geo métrica, se a sua densidade é dada por

$$
f_{X}(x)=f_{X}(x ; p)= \begin{cases}p(1-p)^{x} & \text { para } x=0,1,2, \ldots \\ 0 & \text { para outros valores }\end{cases}
$$

$\operatorname{com} 0<\mathrm{p} \leq 1$ e $\mathrm{q}=1-\mathrm{p}$ 


\subsubsection{2 - Função geradora de momentos}

$$
m_{X}(t)=\frac{p}{1-q e^{t}}
$$

pois,

$$
\begin{aligned}
& m_{X}(t)=E\left[e^{t X]}=\sum\left[e^{t x} p(1-p)^{x}\right]\right. \\
& m_{X}(t)=\sum_{x=0}^{\infty} e^{t x} p(1-p)^{x}=p \sum_{x=0}^{\infty} e^{t X}(1-p)^{x} \\
& m_{X}(t)=p \sum\left(q e^{t}\right)^{x} \\
& m_{X}(t)=p\left[1+q e^{t}+\left(q e^{t}\right)^{2}+\left(q e^{t}\right)^{3}+\ldots\right]
\end{aligned}
$$

como $\frac{1}{1-x}=\sum_{k=0}^{\infty} \frac{k ! x^{K}}{k !}=\sum_{k=0}^{\infty} x^{k},|x|<1$, pelo desenvolvimento da série de Taylor, tem-se que

$$
\begin{aligned}
& \mathrm{m}_{\mathrm{X}}(\mathrm{t})=\mathrm{p} \cdot \frac{1}{1-q e^{\mathrm{t}}} \quad ; \quad\left|\mathrm{qe} \mathrm{t}^{\mathrm{t}}\right|<1 \\
& \mathrm{~m}_{\mathrm{X}}(\mathrm{t})=\frac{\mathrm{p}}{1-\mathrm{qe}}
\end{aligned}
$$

3.4.1.5.3 - Cālculo dos principais momentos pela f.g.m.

$$
\mu_{1}^{\prime}=E(X)=\frac{q}{p}
$$

pois, 


$$
\begin{aligned}
& \mathrm{m}_{X}^{\prime}(t)=p q e^{t}\left(1-q e^{t}\right)^{-2} \\
& m_{X}^{\prime}(0)=\frac{p q}{p^{2}}=\frac{q}{p} \\
& \mu_{2}^{\prime}=E\left(X^{2}\right)=\frac{q}{p}+2 \frac{q^{2}}{p^{2}}
\end{aligned}
$$

pois,

$$
\begin{aligned}
& \mathrm{m}_{\mathrm{X}}^{\prime \prime}(\mathrm{t})= \mathrm{pqe} \mathrm{e}^{\mathrm{t}}\left(1-q \mathrm{e}^{\mathrm{t}}\right)^{-2}+2 \mathrm{p}\left(\mathrm{qe} \mathrm{e}^{\mathrm{t}}\right)^{2}\left(1-q \mathrm{e}^{\mathrm{t}}\right)^{-3} \\
& \mathrm{~m}_{\mathrm{X}}^{\prime \prime}(0)=\frac{\mathrm{pq}}{\mathrm{p}^{2}}+\frac{2 \mathrm{pq}^{2}}{\mathrm{p}^{3}}=\frac{\mathrm{q}}{\mathrm{p}}+\frac{2 \mathrm{q}^{2}}{\mathrm{p}^{2}} \\
& \mu_{2}=\operatorname{Var}(\mathrm{X})=\frac{\mathrm{q}}{\mathrm{p}^{2}}
\end{aligned}
$$

pois,

$$
\begin{aligned}
& \mu_{2}=\mu_{2}^{\prime}-\mu_{1}^{2} \\
& \mu_{2}=\frac{q}{p}+\frac{2 q^{2}}{p^{2}}-\frac{q^{2}}{p^{2}}=\frac{q}{p}+\frac{q^{2}}{p^{2}} \\
& \mu_{2}=\frac{q p+q^{2}}{p^{2}}=\frac{q(p+q)}{p^{2}}=\frac{q}{p^{2}}
\end{aligned}
$$

\subsubsection{6 - Distribuição binomial negativa}

\subsubsection{1-Definição}

Def inese uma V.A. discreta X, tendo distribuição bi nomial negativa, se a sua densidade é dada por

$$
f_{X}(x)=f_{X}(x ; r, p)=\left\{\begin{array}{cc}
\left(\begin{array}{c}
r+x-1 \\
x
\end{array}\right) p^{r} q^{x}= & \left(\begin{array}{c}
-r \\
x
\end{array}\right) p^{r}(-q)^{x}, \text { para } x=0,1,2 \ldots \\
0 \quad & \text { para outros valores }
\end{array}\right.
$$


com $r=1,2,3, \ldots ; 0<p \leq 1 ; q=1-p$

Notas:

(2) Se na distribuição binomial negativa tomar-se $r=1$, obtem-se a função de densidade da distribuição geométrica

$$
f_{X}(x ; 1, p)=p(1-p)^{x} \quad, \quad \text { para } x=0,1,2,3, \ldots
$$

(3) Essa distribuição recebe o nome de binomial negativa pelo fato de $f_{X}(x)$ ser o termo geral da expansão de

$$
p^{r}[1-(1-p)]^{-r}
$$

$$
\begin{aligned}
& 3.4 .1 .6 .2 \text { - Função geradora de momentos } \\
& \mathrm{m}_{X}(t)=\left(\frac{p}{1-q e^{t}}\right)^{r}
\end{aligned}
$$

pois,

$$
\begin{aligned}
m_{X}(t) & =E\left[e^{t X}\right]=\Sigma\left[e^{t x}\left(\begin{array}{c}
r+x-1 \\
x
\end{array}\right) p^{r} q^{x}\right] \\
& =\Sigma\left[e^{t x}\left(\begin{array}{c}
-r \\
x
\end{array}\right) p^{r}(-q) x\right] \\
& =\sum_{0}^{\infty} e^{t x}\left(\begin{array}{c}
-r \\
x
\end{array}\right) p^{r}(-q)^{x} \\
& =\sum_{0}^{\infty}\left(\begin{array}{c}
-r \\
x
\end{array}\right) p^{r}\left(-q e^{t}\right)^{x} \\
= & p^{r} \sum_{0}^{\infty}\left(\begin{array}{c}
-r \\
x
\end{array}\right)\left(-q e^{t}\right)^{x}
\end{aligned}
$$


49.

$$
\begin{aligned}
& m_{X}(t)=p^{r}\left(1-q e^{t}\right)^{-r} \\
& \mathrm{~m}_{X}(t)=\left[\frac{\mathrm{p}}{1-\mathrm{qe}^{t}}\right]^{\mathrm{r}} \quad, \quad\left|\mathrm{q} \mathrm{e}^{\mathrm{t}}\right|<1 \text { ou } t<-\ell \mathrm{n} \mathrm{q}
\end{aligned}
$$

3.4.1.6.3 - Cálculo dos principais momentos por f.g.m.

$$
\mu_{1}^{\prime}=E(X)=\frac{r q}{p} .
$$

pois,

$$
\begin{aligned}
& m_{X}^{\prime}(t)=p^{r} r q e^{i}\left(1-q e^{t}\right)^{-r-1} \\
& m_{X}^{\prime}(0)=p^{r} r q(1-q)^{-r-1}=p^{-1} r q=\frac{r q}{p} \\
& \mu_{2}^{\prime}=E\left(X^{2}\right)=\frac{r^{<} q^{2}}{p^{2}}+\frac{r q^{2}}{p^{2}}+\frac{r q}{p}
\end{aligned}
$$

pois,

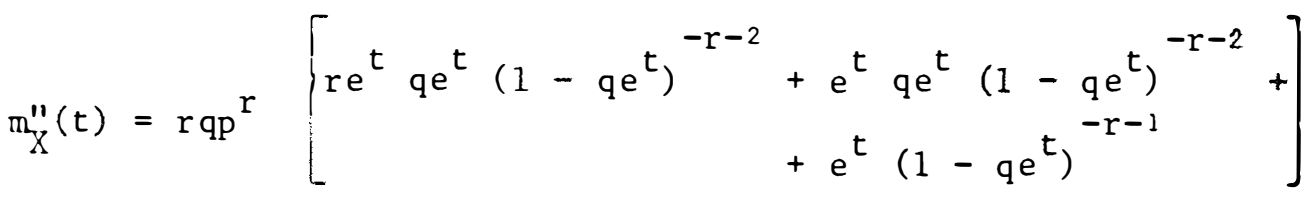

$$
\begin{aligned}
& m_{X}^{\prime \prime}(0)=r q p r\left[r q(1-q)^{-r-2}+q(1-q)^{-r-2}+(1-q)^{-r-1}\right] \\
& \mathrm{m}_{\mathrm{X}}^{\prime \prime}(0)=\mathrm{r}^{2} \mathrm{q}^{2} \mathrm{p}^{-2}+\mathrm{rq}^{2} \mathrm{p}^{-2}+\mathrm{rqp} \mathrm{p}^{-1} \\
& m_{x}^{\prime \prime}(0)=\frac{r^{2} q^{2}}{p^{2}}+\frac{r q^{2}}{p^{2}}+\frac{r q}{p}
\end{aligned}
$$




$$
u_{z}=\operatorname{Var}(\mathrm{X})=\frac{\mathrm{rq}}{\mathrm{p}^{2}}
$$

pois,

$$
\begin{aligned}
& \mu_{2}=\mu_{2}^{\prime}-u_{i}^{2} \\
& u_{2}=\frac{r^{2} q^{2}}{p^{2}}+\frac{r q^{2}}{p^{2}}+\frac{r q}{p}-\frac{r^{2} q^{2}}{p^{2}} \\
& \mu_{2}=\frac{r q}{p}\left(\frac{q+p}{p}\right)=\frac{r q}{p^{2}}
\end{aligned}
$$

\subsubsection{7 - Distribuiçäo multinomial}

\subsubsection{1 - Definição}

Suponha-se que os eventos mutuamente exclusivos $A_{1}$, $A_{2}, \ldots, A_{K}$ podem ocorrer com probabilidades $P_{1}, P_{2}, \ldots, P_{K}$, tal que $\sum_{j=1} p_{j}=1$. Se $x_{1}, x_{2}, \ldots, x_{K}$ são as V.A. que dão, respectivamente, os nümeros de vezes que os eventos $A_{j}$ ocorrem em $\underline{n}$ provas in dependentes, de modo que $x_{1}+x_{2}+\ldots+x_{K}=n$, a probabilidade de que ocorram $x_{1}$ eventos $A_{1}, x_{2}$ eventos $A_{2}, \ldots, x_{K}$ eventos $A_{K}$ em $\underline{n}$ provas, tem distribuição multinomial de probabilidade com função de probabilidade conjunta dada por

$$
f_{x_{1}}, \ldots, x_{K}\left(x_{1}, x_{2}, \ldots, x_{K}\right)=\frac{n !}{x_{1} ! x_{2} ! \ldots x_{K} !} p_{1}^{x_{1}} p_{2}^{x_{2}} \ldots p_{K}^{x_{K}}
$$

Essa distribuição é uma generalização da distribuição binomial, e recebe o nome de distribuição multinomial por corresponder ao termo geral do desenvolvimento multinomial de

$$
\left(p_{1}+p_{2}+\ldots+p_{K}\right)^{n} \text {. }
$$




\subsubsection{2 - Função geradora de momentos}

$$
m_{x_{1}}, \ldots, x_{K}\left(t_{1}, \ldots, t_{K}\right)=\left(p_{1} e^{t_{1}}+\ldots+p_{K} e^{t_{k}}\right)^{n}
$$

pois,

$$
\begin{aligned}
& m_{x_{1}}, \ldots, x_{k}\left(t_{1}, \ldots, t_{k}\right)=E\left[e^{\sum_{j=1}^{k} t_{j} x_{j}}\right] \\
& =\Sigma\left[e^{t_{1} x_{1}+\ldots+t_{K} x_{k}} \frac{n !}{x_{1} ! x_{2} ! \ldots x_{K} !} p_{1}^{x_{1}} \ldots p_{K}^{x_{K}}\right]
\end{aligned}
$$

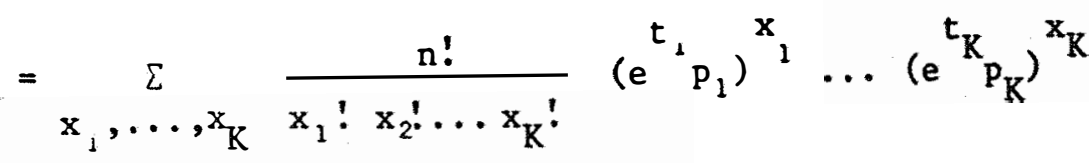

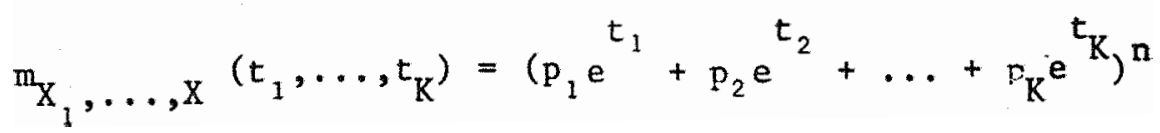$$
\text { 3.4.1.7.3 - Cālculo dos principais momen- }
$$$$
\text { tos por f.g.m. }
$$$$
\mu_{1}^{\prime}=E\left(x_{j}\right)=n p_{j}
$$

pois,

$$
\begin{aligned}
E\left(x_{i}\right) & =\frac{\partial}{\partial t_{i}} m_{X_{1}}, \ldots,\left.x_{k}\left(t_{1}, \ldots, t_{K}\right)\right|_{t_{1}=t_{2}=\ldots=t_{K}=0} \\
& =\left.n\left(\Sigma p_{j} e^{t} j\right)^{n-1} p_{i} e^{t}\right|_{t_{1}=t_{2}=\ldots=t_{K}=0} \\
& =n \cdot 1 \cdot p_{i} \\
& =n p_{i}
\end{aligned}
$$


de forma que: $E\left(X_{1}\right)=n p_{1}$

$$
\begin{gathered}
E\left(X_{2}\right)=n p_{2} \\
\cdots \\
E\left(X_{K}\right)=n
\end{gathered}
$$

$$
\mu_{2}^{\prime}=E\left(x_{i}^{2}\right)=n^{2} p_{i}^{2}-n p_{i}^{2}+n p_{i}
$$

pois,

$$
\begin{aligned}
E\left(x_{i}^{2}\right) & =\frac{\partial^{2}}{\partial t_{i}^{2}} m_{x_{1}}, \ldots,\left.x_{K}\left(t, \ldots, t_{K}\right)\right|_{t_{1}=t_{2}=\ldots=t_{k}=0} \\
& =\left.n\left[(n-1)\left(\sum p_{j} e^{t}\right)^{n-2} p_{i}^{2} e^{<t_{i}}+\left(\sum p_{j} e^{t}\right)^{n-1} p_{i} e^{t_{i}}\right]\right|_{t_{1}=\ldots=t_{k}=0} \\
& =n(n-1) p_{i}^{2}+n p_{i} \\
& =n^{2} p_{i}^{2}-n p_{i}^{2}+n p_{i} \\
\mu_{2} & =\operatorname{Var}\left(x_{i}\right)=n p_{i}\left(1-p_{i}\right)
\end{aligned}
$$

pois,

$$
\begin{aligned}
& \mu_{2}=\mu_{2}^{\prime}-\mu_{1}^{2} \\
& \mu_{2}=n^{2} p_{i}^{2}-n p_{i}^{2}+n p_{i}-n^{2} p_{i}^{2} \\
& \mu_{2}=n p_{i}\left(1-p_{i}\right)=n p_{i} q_{i}
\end{aligned}
$$

de forma que: $\operatorname{Var}\left(x_{1}\right)=n p_{1}\left(1-p_{1}\right)$

$$
\begin{gathered}
\operatorname{Var}\left(\mathrm{X}_{2}\right)=n \mathrm{p}_{2}\left(1-\mathrm{p}_{2}\right) \\
\ldots \\
\operatorname{Var}\left(\mathrm{X}_{\mathrm{K}}\right)=n \mathrm{p}_{\mathrm{K}}\left(1-\mathrm{p}_{\mathrm{K}}\right)
\end{gathered}
$$




$$
E\left(X_{1}, X_{2}, \ldots, X_{K}\right)=n(n-1) \ldots(n-i+1) p_{1} p_{2} \ldots p_{K}
$$

pois,

$$
\begin{aligned}
E\left(X_{1}, X_{2}, \ldots, x_{K}\right) & =\frac{\partial^{i}}{\partial t_{1} \ldots \partial t_{K}} m_{x_{1}}, \ldots,\left.x_{K}\left(t_{1}, \ldots, t_{K}\right)\right|_{t_{1}=\ldots=t_{K}=0} \\
& =\left.n(n-1) \ldots(n-i+1) p_{1} \ldots p_{K}\left(\Sigma p_{j} e^{t} j\right)^{n-i}\right|_{t_{1}=\ldots=t_{K}=0} \\
& =n(n-1) \ldots(n-i+1) p_{1} p_{2} \ldots p_{K} .
\end{aligned}
$$

3.4.2 - Distribuições continuas

3.4.2.1 - Distribuição retangular (ou uniforme)

$$
\text { 3.4.2.1.1 - Definição }
$$

Define-se uma variāvel aleatória continua $\mathrm{X}$, tendo distribuição retangular (ou uniforme), se a sua densidade de probabilidade é dada por

$$
f_{X}(x)=f_{X}(x ; a, b)=\frac{1}{b-a} \quad, \quad I_{[a, b]}(x)
$$

com $-\infty<a<b<+\infty$.

3.4.2.1.2 - Função geradora de momentos

$$
m_{X}(t)=\frac{e^{b t}-e^{a t}}{(b-a) t}
$$


54.

pois,

$$
\begin{aligned}
m_{X}(t) & =E\left[e^{t x}\right] \\
& =\int_{a}^{b} e^{t x} \frac{1}{b-a} d x \\
& =\frac{1}{b-a} \int_{a}^{b} e^{t x} d x=\frac{1}{b-a}\left[\frac{1}{t} e^{t x}\right]_{a}^{b} \\
& =\frac{e^{b t}-e^{a t}}{(b-a) t}
\end{aligned}
$$

\subsubsection{3 - Cátculo dos principais momen-} tos por f.g.m.

$$
\mu_{1}^{\prime}=E(X)=\frac{a+b}{2}
$$

pois,

$$
\begin{aligned}
\mathrm{m}_{X}^{\prime}(t) & =\frac{e^{t b}[t b-1]-e^{a t}[t a-1]}{(b-a) t^{2}} \\
& =\frac{\left[1+b t+\frac{(b t)^{2}+(b t)^{3}}{2 !}+\ldots\right](b t-1)-\left[1+a t+\frac{(a t)^{2}}{2 !}+\ldots\right](a t-1)}{(b-a) t^{2}} \\
& =\frac{\left(\frac{b^{2}-a^{2}}{2}\right)+t\left(\frac{2 b^{3}-2 a^{3}}{6}\right)+t^{2}(\ldots)+t^{3}(\ldots)+\ldots}{(b-a)}
\end{aligned}
$$

$$
m_{x}^{\prime}(0)=\frac{\frac{b^{2}-a^{2}}{2}}{b-a}=\frac{a+b}{2}
$$




$$
\text { Observe-se que o cálculo dos momentos, pela f.g.m., }
$$
para a distribuição retangular, é um processo exaustivo, porém, os principais momentos podem ser obtidos de imediato pela aplicação da pröpria definição, ou seja,

$$
\begin{aligned}
& \mu_{1}^{\prime}=E(X)=\int_{a}^{b} x \frac{1}{b-a} d x=\frac{a+b}{2} \\
& \mu_{2}^{\prime}=E\left(X^{2}\right)=\int_{a}^{b} x^{2} \frac{1}{b-a} d x=\frac{b^{2}+a b+a^{2}}{3} \\
& y_{2}=\operatorname{Var}(X)=E\left(X^{2}\right)-[E(X)]^{2}=\frac{(b-a)^{2}}{12}
\end{aligned}
$$

Nota (4) - A distribuição uniforme pode também ser definida no intervalo aberto $(a, b)$ ou misto $[a, b)$ ou $(a, b]$.

\subsubsection{2 - Distribuição exponencial (negativa)}

\subsubsection{1 - Definição}

Define-se uma V.A. contînua $X$, tendo distribuição ex ponencial (negativa) se a sua densidade de probabilidade é dada por:

$$
\mathrm{f}_{X}(\mathrm{x})=\mathrm{f}_{\mathrm{X}}(\mathrm{x} ; \lambda)=\lambda \mathrm{e}^{-\lambda \mathrm{x}}, \mathrm{I}_{[0, \infty)}(\mathrm{x})
$$

$\operatorname{com} \lambda>0$ 


$$
\begin{aligned}
& 3.4 .2 .2 .2 \text { - Função geradora de momentos } \\
& m_{x}(t)=\frac{\lambda}{\lambda-t},
\end{aligned}
$$

pois,

$$
\begin{aligned}
m_{X}(t)=E\left[e^{t x}\right] & =\int_{0}^{\infty} e^{t x} \lambda e^{-\lambda x} d x \\
& =\lambda \int_{0}^{\infty} e^{x(t-\lambda)} d x=\lambda \int_{0}^{\infty} e^{-u} \frac{d u}{-t+\lambda}
\end{aligned}
$$

substituindo-se: $u=-x(t-\lambda), \frac{d u}{-t+\lambda}=d x$,

$$
m_{X}(t)=\frac{\lambda}{\lambda-t} \int_{0}^{\omega} e^{-u} d u
$$

e, sendo $\Gamma(1)=\int_{0}^{\infty} e^{-u} d u=1$, tem-se que

$$
\mathrm{m}_{\mathrm{X}}(\mathrm{t})=\frac{\lambda}{\lambda-\mathrm{t}} \quad, \quad \mathrm{t}<\lambda .
$$

\subsubsection{3 - Cầculo dos principais momen-} tos por f.g.m.

$$
\mu_{i}^{\prime}=E(X)=\frac{1}{\lambda}
$$

pois,

$$
\begin{aligned}
& m_{X}^{\prime}(t)=\frac{\lambda}{(\lambda-t)^{2}} \\
& m_{X}^{\prime}(0)=\frac{\lambda}{\lambda^{2}}=\frac{1}{\lambda}
\end{aligned}
$$


57.

$$
\mu_{2}^{\prime}=E\left(X^{2}\right)=\frac{2}{\lambda^{2}}
$$

pois ,

$$
\begin{aligned}
& m_{X}^{\prime \prime}(t)=\frac{2 \lambda(\lambda-t)}{(\lambda-t)^{4}} \\
& m_{0}^{\prime \prime}(t)=\frac{2 \lambda^{2}}{\lambda^{4}}=\frac{2}{\lambda^{2}} \\
& \mu_{2}=\operatorname{Var}(X)=\frac{1}{\lambda^{2}}
\end{aligned}
$$

pois,

$$
\begin{aligned}
& y_{2}=u_{2}^{\prime}-y_{1}^{2} \\
& \mu_{2}=\frac{2}{\lambda^{2}}-\frac{1}{\lambda^{2}}=\frac{1}{\lambda^{2}}
\end{aligned}
$$

3.4.2.3 - Distribuição gama

3.4.2.3.1 - Definição

Define-se uma V.A. continua $x$, tendo distribuição ga ma, se a sua densidade de probabilidade é dada por

$$
\begin{aligned}
& \mathrm{f}_{X}(\mathrm{x})=\mathrm{f}_{\mathrm{X}}(\mathrm{x} ; \mathrm{r}, \lambda)=\frac{\lambda}{\Gamma(\mathrm{r})}(\lambda \mathrm{x})^{\mathrm{r}-1} \mathrm{e}^{-\lambda \mathrm{x}}, \mathrm{I}_{[0, \infty)}(\mathrm{x}) \\
& \text { com } \mathrm{r}>0 \text { e } \lambda>0
\end{aligned}
$$

3.4.2.3.2 - Função geradora de momentos

$$
\mathrm{m}_{X}(t)=\left(\frac{\lambda}{\lambda-t}\right)^{r},
$$


58.

pois,

$$
\begin{aligned}
\mathrm{m}_{\mathrm{X}}(\mathrm{t}) & =E\left[e^{t x}\right]=\int_{0}^{\infty} e^{t x} \frac{\lambda}{\Gamma(r)}(\lambda x)^{r-1} e^{-\lambda x} d x \\
& =\frac{\lambda}{\Gamma(r)} \int_{0}^{\infty} e^{t x}(\lambda x)^{r-1} e^{-\lambda x} d x \\
& =\frac{\lambda}{\Gamma(r)} \int_{0}^{\infty} e^{-x(\lambda-t)} x^{r-1} \lambda^{r-1} d x \\
& =\frac{\lambda^{r}}{\Gamma(r)} \int_{0}^{\infty} e^{-x(\lambda-t)} x^{r-1}(\lambda-t)^{r-1} \frac{(\lambda-t)}{(\lambda-t)^{r}} d x \\
& =\frac{\lambda^{r}}{(\lambda-t)^{r}} \frac{1}{\Gamma(r)}(\lambda-t) \int_{0}^{\infty} e^{-x(\lambda-t)}[x(\lambda-t)]^{r-1} d x
\end{aligned}
$$

$$
\begin{aligned}
& \text { substituindo-se: } u=x(\lambda-t) ; \frac{d u}{\lambda-t}=d x, \\
& m_{X}(t)=\left(\frac{\lambda}{\lambda-t}\right)^{r} \frac{1}{\Gamma(r)} \frac{1}{r} r \int_{0}^{\infty} e^{-u} u^{r-1} d u \\
& \text { e como } r \int_{0}^{\infty} e^{-u} u^{r-1} d u=\Gamma(r+1)=r \Gamma(r) \\
& m_{X}(t)=\left(\frac{\lambda}{\lambda-t}\right)^{r} \frac{r \Gamma(r)}{r \Gamma(r)} \\
& m_{X}(t)=\left(\frac{\lambda}{\lambda-t}\right)^{r}
\end{aligned}
$$

3.4.2.3.3 - Cálculo dos principais momentos pela f.g.m.

$$
\mu_{1}^{\prime}=E(X)=\frac{r}{\lambda}
$$


pois,

$$
\begin{aligned}
& m_{X}^{\prime}(t)=r \lambda^{r}(\lambda-t)^{-r-1} \\
& m_{X}^{\prime}(0)=r \lambda^{r} \lambda^{-r-1}=r \lambda^{-1}=\frac{r}{\lambda} \\
& \mu_{2}^{\prime}=E\left(X^{2}\right)=\frac{r(r+1)}{\lambda^{2}}
\end{aligned}
$$

pois,

$$
\begin{aligned}
m_{X}^{\prime \prime}(t) & =r(r+1) \lambda^{r}(\lambda-t)^{-r-2} \\
m_{x}^{\prime \prime}(0)= & r(r+1) \lambda^{r} \lambda^{-r-2}=\frac{r(r+1)}{\lambda^{2}} \\
\mu_{2} & =\operatorname{Var}(X)=\frac{r}{\lambda^{2}}
\end{aligned}
$$

pois,

$$
\begin{aligned}
& \mu_{2}=\mu_{2}^{\prime}-\mu_{1}^{2} \\
& \mu_{2}=\frac{r(r+1)}{\lambda^{2}}-\frac{r^{2}}{\lambda^{2}}=\frac{r^{2}+r-r^{2}}{\lambda^{2}}=\frac{r}{\lambda^{2}}
\end{aligned}
$$

\subsubsection{4 - Distribuição beta}

\subsubsection{1 - Definição}

Define-se uma V.A. contínua $x$, tendo distribuição be ta, se a sua densidade de probabilidade é dada por:

$$
f_{X}(x)=f_{X}(x ; p, q)=\frac{1}{\beta(p, q)} x^{p-1}(1-x)^{q-1}, I_{(0,1)}(x)
$$

$\operatorname{com} p>0$ e $q>0$. 
A função geradora de momentos para essa distribuição não é usada, pois a mesma não tem uma forma simples (ver quadro 2, de acordo com LUKACS, 1970), e o cálculo dos principais momentos po de ser feito de imediato pela utilização de suas definições, e mais precisamente a partir da seguinte igualdade:

$$
E\left(X^{K}\right)=\frac{\Gamma(k+p) \Gamma(p+q)}{\Gamma(p) \Gamma(k+p+q)} \text { * }
$$

Demonstração:

$$
\begin{aligned}
E\left(X^{K}\right) & =\frac{1}{\beta(p, q)} \int_{0}^{1} x^{k+p-1}(1-x)^{q-1} d x \\
& =\frac{1}{\beta(p, q)} \beta(k+p, q)=\frac{\Gamma(k+p) \Gamma(q) \Gamma(p+q)}{\Gamma(k+p+q) \Gamma(p) \Gamma(q)} \\
& =\frac{\Gamma(k+p) \Gamma(p+q)}{\Gamma(p) \Gamma(k+p+q)}
\end{aligned}
$$

Obtem-se facilmente, então, que:

$$
\begin{aligned}
& \mu_{1}^{\prime}=E(X)=\frac{p}{p+q} \\
& \mu_{2}^{\prime}=E\left(X^{2}\right)=\frac{p(p+1)}{(p+q)(p+q+1)} \\
& \mu_{2}=\operatorname{Var}(X)=\frac{p q}{(p+q)^{2}(p+q+1)}
\end{aligned}
$$


61.

\subsubsection{5 - Distribuição normal}

\subsubsection{1 - Definição}

Define-se uma V.A. contínua $x$, tendo distribuição nor mal, se a sua densidade de probabilidade é dada por

$$
f_{X}(x)=f_{X}\left(x ; m, \sigma^{2}\right)=\frac{1}{\sigma \sqrt{2 \pi}} e^{-\left[(x-m)^{2} /\left(2 \sigma^{2}\right)\right]} \text {, para }-\infty<x<\infty
$$

com $-\infty<\mathrm{m}<\infty$ e $\sigma>0$, sendo $\mathrm{m}=$ mëdia, $\sigma^{2}=$ variância e $\sigma=$ = desvio padräo.

3.4.2.5.2 - Função geradora de momentos

$$
m_{X}(t)=e^{t m+\left[\left(\sigma^{2} t^{2}\right) / 2\right]}
$$

pois,

$$
\begin{aligned}
\mathrm{m}_{X}(t) & =E\left[e^{t x}\right]=\int_{-\infty}^{+\infty} e^{t x} \frac{1}{\sigma \sqrt{2 \pi}} e^{-\left[(x-m)^{2} /\left(2 \sigma^{2}\right)\right]} d x \\
& =\frac{1}{\sigma \sqrt{2 \pi}} \int_{-\infty}^{+\infty} e^{t x} e^{-\left[(x-m)^{2} /\left(2 \sigma^{2}\right)\right]} d x
\end{aligned}
$$

substituindo-se:

$$
\begin{array}{rlrl}
\frac{x-m}{\sigma} & =u & \text { e, se } x \rightarrow-\infty \rightarrow u \rightarrow-\infty \\
x \rightarrow+\infty \rightarrow u \rightarrow+\infty & \\
x & =\sigma u+m \\
d x & =\sigma d u & &
\end{array}
$$


62.

$$
\begin{aligned}
m_{X}(t) & =\frac{1}{\sqrt{2 \pi}} \int_{-\infty}^{+\infty} e^{-\left[(1 / 2) u^{2}\right]+t(\sigma u+m)} d u \\
& =\frac{e^{t m}}{\sqrt{2 \pi}} \int_{-\infty}^{+\infty} e^{-\left[(1 / 2) u^{2}\right]+t \sigma u} d u \\
& =\frac{e^{t m}}{\sqrt{2 \pi}} \int_{-\infty}^{+\infty} e^{-(1 / 2)\left[\left(u^{2}-2 t \sigma u\right)\right]} d u \\
& =\frac{e^{t m}}{\sqrt{2 \pi}} \int_{-\infty}^{+\infty} e^{-(1 / 2)\left[(u-\sigma t)^{2}-\sigma^{2} t^{2}\right]} d u \\
& =\frac{e^{t m}}{\sqrt{2 \pi}} e^{\left(\sigma^{2} t^{2}\right) / 2} \int_{-\infty}^{+\infty} e^{-(1 / 2)\left[(u-\sigma t)^{2}\right]} d u
\end{aligned}
$$

substituindo-se, novamente,

$$
\begin{aligned}
u-\sigma t & =v \\
u & =v+\sigma t \\
d u & =d v,
\end{aligned}
$$

tem-se que:

$$
m_{X}(t)=e^{t m+\left(\sigma^{2} t^{2}\right) / 2} \frac{1}{\sqrt{2 \pi}} \int_{-\infty}^{+\infty} e^{-\left(v^{2} / 2\right)} d v
$$

e, sendo $\frac{1}{\sqrt{2 \pi}} \int_{-\infty}^{+\infty} e^{-\left(v^{2} / 2\right) d v}=1$ (distribuição normal reduzida), obtem-se finalmente a f.g.m.

$$
m_{X}(t)=e^{t m+\left(\sigma^{2} t^{2}\right) / 2}
$$

Dessa forma, a função geradora de momentos para a distribuição nornal è dada por: 
63.

$$
\begin{array}{ll}
m_{X}(t)=e^{t m+\left(\sigma^{2} t^{2}\right) / L} & , \text { para } x \cap N\left(m, \sigma^{2}\right) \\
m_{X}(t)=e^{\left(\sigma^{2} t^{2}\right) / 2} & , \text { para } x \cap N\left(0, \sigma^{2}\right) \\
m_{X}(t)=e^{t^{2} / 2} & , \text { para } x \cap N(0,1)
\end{array}
$$

3.4.2.5.3 - Càlculo dos principais momentos pela f.g.m.

$$
\mu_{1}^{\prime}=E(X)=m
$$

pois,

$$
\begin{aligned}
& m_{X}^{3}(t)=\left(m+\sigma^{2} t\right) e^{t m+\left(\sigma^{2} t^{2}\right) / 2} \\
& m_{X}^{\prime}(0)=m e^{0}=m \\
& \mu_{2}^{\prime}=E\left(X^{2}\right)=\sigma^{2}+m^{2}
\end{aligned}
$$

pois,

$$
\begin{aligned}
m_{X}^{\prime \prime}(t)=e^{t m+\left(\sigma^{2} t^{2}\right) / 2}\left[\sigma^{2}+\left(m+\sigma^{2} t\right)^{2}\right] \\
m_{X}^{\prime \prime}(0)=e^{0}\left[\sigma^{2}+m^{2}\right]=\sigma^{2}+m^{2} \\
\mu_{2}=\operatorname{var}(X)=\sigma^{2}
\end{aligned}
$$

pois,

$$
\begin{aligned}
& \mu_{2}=\mu_{2}^{\prime}-\mu_{1}^{2}= \sigma^{2}+m^{2}-m^{2}=\sigma^{2} \\
& \mu_{3}^{\prime}=E\left(X^{3}\right)=3 m \sigma^{2}+m^{3}
\end{aligned}
$$

pois, 


$$
\begin{gathered}
\mathrm{m}_{X}^{\prime \prime \prime}(t)=\left(\mathrm{m}+t \sigma^{2}\right) e^{t \mathrm{~m}+\left(t^{2} \sigma^{2}\right) / 2}\left[3 \sigma^{2}+\left(m+t \sigma^{2}\right)^{2}\right] \\
\mathrm{m}_{X}^{\prime \prime \prime}(0)=\mathrm{m} \mathrm{e}^{0}\left[3 \sigma^{2}+\mathrm{m}^{2}\right]=3 \mathrm{~m} \sigma^{2}+\mathrm{m}^{3} \\
\mu_{3}=0
\end{gathered}
$$

pois,

$$
\begin{aligned}
& \mu_{3}=\mu_{3}^{\prime}-3 \mu_{2} \mu_{1}^{3}-\mu_{1}^{3} \\
& \mu_{3}=3 m \sigma^{2}+m^{3}-3 \sigma^{2} m-m^{3}=0 \\
& \mu_{4}^{3}=E\left(X^{4}\right)=3 \sigma^{4}+6 m^{2} \sigma^{2}+m^{4}
\end{aligned}
$$

pois,

$$
\begin{aligned}
& m_{X}^{\prime \prime \prime \prime}(t)= e^{t m+\left(\sigma^{2} t^{2}\right) / 2}\left\{\begin{array}{r}
3 \sigma^{4}+\sigma^{2}\left(m+t \sigma^{2}\right)^{2}+\left(m+t \sigma^{2}\right)^{2}\left[3 \sigma^{2}+\left(m+t \sigma^{2}\right)^{2}+\right. \\
+2 \sigma^{2}\left(m+t \sigma^{2}\right)^{2}
\end{array}\right\} \\
& m_{X}^{\prime \prime \prime}(0)=e^{0}\left\{3 \sigma^{4}+\sigma^{2} m^{2}+m^{2}\left[3 \sigma^{2}+m^{2}\right]+2 \sigma^{2} m^{2}\right\} \\
&=3 \sigma^{4}+6 m^{2} \sigma^{2}+m^{4} \\
& \mu_{4}=3 \sigma^{4}
\end{aligned}
$$

pois,

$$
\mu_{4}=\mu_{4}^{\prime}-4 \mu_{3} \mu_{1}^{\prime}-6 \mu_{2} \mu_{1}^{2}-\mu_{1}^{\prime}
$$




\subsubsection{4 - Fórmula recorrente para cāi- culo de momentos de ordem par}

A distribuição normal apresenta uma interessante för mula recorrente para cálculo de momentos de ordem par, que muito fa cilita o cálculo.

1) Se $x \cap N(0,1)$, então

$$
\begin{gathered}
\mu_{2 n+1}=0 \quad \text { e } \quad \mu_{2 n}=(2 n-1) \mu_{2 n-2}=\frac{(2 n) !}{2^{n}(n) !} \\
\text { para } n=1,2,3,4, \ldots
\end{gathered}
$$

Demonstra-se inicialmente que

$$
\begin{aligned}
& \mu_{2 n+1}=E\left[X^{2 n+1}\right]=0 \\
& \text { De fato, } \\
& E\left[X^{2 n+1}\right]=\frac{1}{\sqrt{2 \pi}} \int_{-\infty}^{+\infty} x^{2 n+1} e^{-x^{2} / 2} d x \\
& =\frac{1}{\sqrt{2 \pi}}\left[\int_{-\infty}^{0} x^{2 n+1} e^{-x^{2} / 2} d x+\int_{0}^{\infty} x^{2 n+1} e^{-x^{2} / 2} d x\right] \\
& =\frac{1}{\sqrt{2 \pi}}\left[\int_{-\infty}^{0} y^{2 n+1} e^{-y^{2} / 2} d y+\int_{0}^{\infty} x^{2 n+1} e^{-x^{2} / 2} d x\right] \\
& =\frac{1}{\sqrt{2 \pi}}\left[-\int_{0}^{\infty} x^{2 n+1} e^{-x^{2} / 2} d x+\int_{0}^{\infty} x^{2 n+1} e^{-x^{2} / 2} d x\right] \\
& =0
\end{aligned}
$$


o que corresponde a afirmar que

$$
u_{2 n+1}=E\left[X^{2 n+1}\right]=0
$$

e, em particular, que

$$
E[X]=0 \text {. }
$$

A seguir, demonstra-se que

$$
\mu_{2 n}=E\left[X^{2 n}\right]=\frac{(2 n) !}{2^{n}(n) !}=(2 n-1) \mu_{2 n-2}
$$

De fato,

$$
\begin{aligned}
\mu_{2 n}=E\left[X^{2 n}\right] & =\frac{1}{\sqrt{2 \pi}} \int_{-\infty}^{+\infty} x^{2 n} e^{-x^{2} / 2} d x=\frac{1}{\sqrt{2 \pi}} 2 \int_{0}^{\infty} x^{2 n} e^{-x^{2} / 2} d x \\
& =\frac{2}{\sqrt{2 \pi}}\left[-\int_{0}^{\infty} x^{2 n-1} \mathrm{de}^{-x^{2} / 2}\right]
\end{aligned}
$$

e, integrando por partes,

$$
\begin{aligned}
& =\frac{2}{\sqrt{2 \pi}}\left[-\left.x^{2 n-1} e^{-x^{2} / 2}\right|_{0} ^{\infty}+(2 n-1) \int_{0}^{\infty} x^{2 n-2} e^{-x^{2} / 2} d x\right] \\
& =(2 n-1) \frac{2}{\sqrt{2 \pi}} \int_{0}^{\infty} x^{2 n-2} e^{-x^{2} / 2} d x \\
& =(2 n-1) \frac{1}{\sqrt{2 \pi}} \int_{-\infty}^{+\infty} x^{2 n-2} e^{-x^{2} / 2} d x \\
& =(2 n-1) \mu(2 n-2)
\end{aligned}
$$


tem-se entao que

$$
\mu_{2 n}=(2 n-1) \mu_{(2 n-2)}
$$

de forma que

$$
\begin{aligned}
\mu_{2 n} & =(2 n-1) \mu_{2 n-2} \\
\mu_{2 n-2} & =(2 n-3) \mu_{2 n-4} \\
\ldots & \cdots \\
\mu_{2} & =1 \mu_{0} \\
\mu_{0} & =1
\end{aligned}
$$

e, multiplicando-se sucessivamente cada qual pelo seu valor correspond ente, tem-se

$$
\begin{aligned}
\mu_{2 n} & =(2 n-1)(2 n-3) \ldots(1) \\
& =\frac{1 \cdot 2 \ldots(2 n-3)(2 n-2)(2 n-1)(2 n)}{2 \ldots(2 n-2)(2 n)} \\
& =\frac{2 \cdot 1 \ldots[2(n-1)](2 n)}{(2 n) !} \\
& =\frac{(n-1) n]}{2^{n}[1 \ldots(2 n) !} \\
& =\frac{(2 n)}{2^{n}(n) !}
\end{aligned}
$$

o que corresponde a afirmar que

$$
\mu_{2 n}=E\left[x^{2 n}\right]=(2 n-1) \mu_{(2 n-2)}=\frac{(2 n) !}{2^{n}(n) !},
$$


68.

e, em particular, que, se

$$
\begin{aligned}
& \mathrm{n}=1, \quad \sigma^{2}(\mathrm{X})=\mathrm{E}\left(\mathrm{X}^{2}\right)=\frac{2 !}{2 ! 1 !}=1 \\
& \mathrm{n}=2, \quad \mu_{4}=\frac{4 !}{4 \cdot 2 !}=3 \\
& \mathrm{n}=3, \quad \mu_{6}=\frac{6 !}{8 \cdot 3 !}=15 \\
& \mathrm{n}=4 \text {, } \\
& \mu_{8}=\frac{8 !}{16.4 !}=105 \\
& \text {. }
\end{aligned}
$$

2) Se $x \cap N\left(m, \sigma^{2}\right)$, então

$$
\mu_{2 n+1}=0 \quad \text { e } \quad \mu_{2 n}=(2 n-1) \sigma^{2} \mu_{(2 n-2)}=\frac{(2 n) !}{2^{n}(n) !} \sigma^{2 n}
$$

Essas igualdades podem ser verificadas por demonstra ções análogas ao caso de $x \cap N(0,1)$, ou pela aplicação da fórmula recorrente (NOGUEIRA, 1965), como apresenta-se a seguir.

$\mathrm{Na}$ förmula recorrente (NOGUEIRA, 1965),

$$
\mu_{N+1}=\sigma^{2} \frac{d}{d m} \mu_{N}+N \sigma^{2} \mu_{N-1}
$$

$$
\begin{aligned}
\text { substituindo-se } \mathrm{N}+1 & =2 \mathrm{n}, \quad \text { para } \mathrm{n}=1,2,3, \ldots \\
\mathrm{N} & =2 \mathrm{n}-1,
\end{aligned}
$$

tem-se

$$
\mu_{2 n}=\sigma^{2} \frac{d}{d m} \mu_{2 n-1}+(2 n-1) \sigma^{2} \mu_{2 n-2}
$$


69.

e, admitindo-se pela simetria da distribuição que

$$
\mu_{2 n-1}=0
$$

obtem-se

$$
\mu_{2 n}=(2 n-1) \quad \sigma^{2} \mu_{2 n-2}
$$

\begin{tabular}{|c|c|c|}
\hline$n=1$ & , & $\mu_{2}=\sigma^{2}$ \\
\hline $\mathrm{n}=2$ & , & $\mu_{4}=3 \sigma^{4}$ \\
\hline$n=3$ & , & $\mu_{6}=15 \sigma^{6}$ \\
\hline$n=4$ & , & $\mu_{8}=105 \sigma^{8}$ \\
\hline . & & $\ldots$ \\
\hline
\end{tabular}

de forma que, se

ou

$$
\mu_{2 n}=\frac{(2 n) !}{2^{n}(n) !} \sigma^{2 n}
$$

de acordo com o item 1 desta secção.

Observe-se que a fórmula recorrente

$$
\mu_{\angle n}=(2 n-1) \mu_{2 n-2}=\frac{(2 n) !}{2^{n}(n) !}
$$

para $x \cap N(0,1)$, pode ser obtida de forma mais imediata também a partir dessa forma recorrente (NOGUEIRA, 1965), bastanto para tanto a substituição de $\sigma^{2}$ por 1 (um). 
3.4.2.6 - Distribuição de qui-quadrado $\left(x^{2}\right)$

\subsubsection{7 - Definição}

Define-se uma V.A. contínua $v_{f}$, tendo distribuição de qui-quadrado, com $f$ graus de liberdade, se a sua densidade de probabilidade è dada por

$$
f_{v_{f}}\left(v_{f}\right)=\frac{1}{2^{f} \frac{1}{2} \Gamma(f / 2)} e^{-v_{f} / 2} v_{f}^{(f-2) / 2}
$$

com $0 \leq \mathrm{v}_{\mathrm{f}} \leq \infty$, onde,

$$
\begin{aligned}
v_{f}=u_{1}^{2}+u_{2}^{2}+\ldots+u_{f}^{2} \quad ; \quad \text { e } \quad u_{i} \cap N(0,1) \\
i=1,2, \ldots, f
\end{aligned}
$$

\subsubsection{2 - Função geradora de momentos}

$$
\mathrm{m}_{\mathrm{f}}(t)=(1-2 t)^{-f / 2}
$$

pois,

$$
m_{v_{f}}(t)=E\left[e^{t v_{f}}\right]=E\left[e^{t\left(u_{1}^{2}+u_{2}^{2}+\ldots+u_{f}^{2}\right)}\right]=E\left[e^{t u_{1}^{2}} e^{t u_{2}^{2}} \ldots e^{t u_{f}^{2}}\right]
$$

e, sendo as variáveis supostas independentes,

$$
\begin{aligned}
m_{v_{f}}(t)=E\left[e^{t v_{f}}\right] & =E\left[e^{t u_{1}^{2}}\right] E\left[e^{t u_{2}^{2}}\right] \ldots E\left[e^{\left.t u_{f}^{2}\right]}\right. \\
\text { Como } u_{i} \cap N(0,1) & E^{\left[t e^{2}\right]}=\frac{1}{\sqrt{2 \pi}} \int_{-\infty}^{+\infty} e^{-u_{i}^{2} / 2} e^{t u_{i}^{2}} d u_{i} \\
& =\frac{1}{\sqrt{2 \pi}} 2 \int_{0}^{\infty} e^{-\left(u_{i}^{2} / 2\right)(1-2 t)} d u_{i}
\end{aligned}
$$


e substituindo

$$
\begin{aligned}
\mathrm{w}^{2} & =\frac{\mathrm{u}^{2}}{2}(1-2 \mathrm{t}) \\
\mathrm{w} & =\mathrm{u} \sqrt{\frac{1-2 t}{2}} \\
\mathrm{dw} & =\sqrt{\frac{1-2 t}{2}} \mathrm{du} \\
\sqrt{\frac{2}{1-2 t} \mathrm{~d}} & =\mathrm{du}
\end{aligned}
$$

tem-se

$$
\begin{aligned}
E\left[e^{\left.t u_{i}^{2}\right]}\right] & =\frac{1}{\sqrt{2 \pi}} 2 \int_{0}^{\infty} e^{-w^{2}} \sqrt{\frac{2}{1-2 t}} d w \\
& =\frac{1}{\sqrt{\pi} \sqrt{1-2 t}} 2 \int_{0}^{\infty} e^{-w^{2}} d w \\
& =\frac{1}{\sqrt{\pi} \sqrt{1-2 t}} \Gamma(1 / 2)=\frac{1}{\sqrt{1-2 t}}
\end{aligned}
$$

ou seja,

$$
E\left[e^{t u^{2}} i\right]=(1-2 t)^{-1 / 2}
$$

e, como $m_{v_{f}}(t)$ é um produto de $\underline{f}$ fatores, obtem-se finalmente que

$$
m_{v_{f}}(t)=(1-2 t)^{-f / 2}
$$


3.4.2.6.3 - Cálculo dos principais momentor por f.g.m.

$$
\mu_{i}^{\prime}=E\left(v_{f}\right)=f
$$

pois,

$$
\begin{aligned}
& m_{v_{f}}^{\prime}(t)=f(1-2 t)^{-(f+2) / 2} \\
& m_{v_{f}}^{\prime}(0)=f
\end{aligned}
$$

$$
\mu_{2}^{\prime}=E\left(v_{f}^{2}\right)=f^{2}+2 f
$$

pois,

$$
\begin{aligned}
& m_{v_{f}^{\prime \prime}}^{\prime \prime}(t)=f(f+2)(1-2 t)^{\left.-\left[\left(f+t_{4}\right) / 2\right)\right]} \\
& m_{v_{f}^{\prime \prime}}^{\prime \prime}(0)=f^{2}+2 f \\
& \mu_{2}=\operatorname{Var}\left(v_{f}\right)=2 f
\end{aligned}
$$

pois,

$$
\begin{aligned}
& \mu_{2}=\mu_{2}^{\prime}-\mu_{1}^{2} \\
& \mu_{2}=f^{2}+2 f-f^{2}=2 f
\end{aligned}
$$

\subsubsection{7 - Distribuição multinormal}

Uma densidade multivariada das mais importantes $\bar{e}$ a densidade normal multivariada (ou multinormal), a qual é uma genera lização da distribuição normal para k variāveis.

Para maior facilidade de aplicação, a distribuição multinormal é apresentada nesse trabalho na forma matricial, bem co 
mo sua função geradora de momentos, de acordo com NOGUEIRA (1968).

\subsubsection{1 - Definição}

Dadas as variāveis aleatörias $x_{i}, i=1,2, \ldots, n$, tal que $x_{i} \cap N\left(0, \sigma_{i}^{2}\right)$, define-se a função densidade de probabilidade mu tinormal a

$$
g_{x_{1}, \ldots, x_{n}}\left(x_{1}, x_{2}, \ldots, x_{n}\right)=\frac{\sqrt{|s|}}{(2 \pi)^{n / 2}} e^{-(1 / 2) x^{\prime} \sigma^{-1} x}
$$

onde:

$$
\begin{aligned}
& \mathrm{X}=\left[\begin{array}{c}
\mathrm{x}_{1} \\
\mathrm{x}_{2} \\
\vdots \\
\mathrm{x}_{\mathrm{n}}
\end{array}\right] \quad \sigma=\left[\begin{array}{cccc}
\sigma_{1}^{2} & \rho_{12} \sigma_{1} \sigma_{2} & \cdots & \rho_{1 \mathrm{n}} \sigma_{1} \sigma_{\mathrm{n}} \\
\rho_{12} \sigma_{1} \sigma_{2} & \sigma_{2}^{2} & \cdots & \rho_{2 \mathrm{n}} \sigma_{2} \sigma_{\mathrm{n}} \\
\vdots & \vdots & \vdots & \vdots \\
\rho_{1 \mathrm{n}} \sigma_{1} \sigma_{\mathrm{n}} & \rho_{2 \mathrm{n}} \sigma_{2} \sigma_{\mathrm{n}} & & \sigma_{\mathrm{n}}^{2}
\end{array}\right] \\
& S=\sigma^{-1} \quad ; \quad|s|=\left|\sigma^{-1}\right|=\frac{1}{|\sigma|} \\
& -\infty<x_{i}<\infty \\
& -1<\rho_{i j}<1 \quad, \quad \rho_{i j} \rightarrow \text { coeficientes de correlação } \\
& \sigma_{i}>0
\end{aligned}
$$

3.4.2.7.1.1 - Função densidade de probabilidade binormal

Define-se duas $V . A$. continuas, $x_{1} \cap N\left(0, \sigma_{1}^{2}\right)$ e $x_{2} \cap N\left(0, \sigma_{2}^{2}\right)$ tendo distribuição binormal de probabilidades, se a sua densidade de 
probabilidade é dada por

$$
g_{X_{1}, X_{2}}\left(x_{1}, x_{2}\right)=\frac{\sqrt{|s|}}{(2 \pi)^{n / 2}} e^{-(1 / 2) X^{\prime} \sigma^{-1} X},
$$

na forma matricial, com

$$
\begin{gathered}
X=\left[\begin{array}{l}
x_{1} \\
x_{2}
\end{array}\right] \quad X^{\prime}=\left[\begin{array}{ll}
x_{1} & x_{2}
\end{array}\right] \\
\sigma=\left[\begin{array}{cc}
\sigma_{1}^{2} & \rho \sigma_{1} \sigma_{2} \\
\rho \sigma_{1} \sigma_{2} & \sigma_{2}^{2}
\end{array}\right] \\
|\sigma|=\frac{\sigma_{1}^{2} \sigma_{2}^{2}}{2}\left(1-\rho^{2}\right) \\
\sigma^{-1}=\frac{1}{\left(1-\rho^{2}\right)}\left[\begin{array}{cc}
\frac{1}{\sigma_{1}^{2}} & \frac{-\rho}{\sigma_{1} \sigma_{2}} \\
\frac{-\rho}{\sigma_{1} \sigma_{2}} & \frac{1}{\sigma_{2}^{2}}
\end{array}\right]
\end{gathered}
$$

tal que

$$
\begin{aligned}
& g_{X_{1}, X_{2}}\left(x_{1}, x_{2}\right)=\frac{1}{2 \pi \sigma_{1} \sigma_{2} \sqrt{1-\rho^{2}}} e^{-1 / 2\left[x_{1} x_{2}\right]\left[1 /\left(1-\rho^{2}\right)\right]}\left[\begin{array}{cc}
1 / \sigma_{1}^{2} & -\rho / \sigma_{1} \sigma_{2} \\
-\rho / \sigma_{1} \sigma_{2} & 1 / \sigma_{2}^{2}
\end{array}\right]\left[\begin{array}{l}
x_{1} \\
x_{2}
\end{array}\right] \\
& g_{X_{1}}, x_{2}\left(x_{1}, x_{2}\right)=\frac{1}{2 \pi \sigma_{1} \sigma_{2} \sqrt{1-\rho^{2}}} e^{-\left[1 /\left\{2\left(1-\rho^{2}\right)\right]\left[\left(x_{1}^{2} / \sigma_{1}^{2}\right)-\left(2 \rho x_{1} x_{2} / \sigma_{1} \sigma_{2}\right)+\left(x_{2}^{2} / \sigma_{2}^{2}\right)\right]\right.} \\
& \text { é a densidade de probabilidade na forma algébrica. }
\end{aligned}
$$




\subsubsection{2 - Funçäo geradora de momentos para a distribuiçăo multi- normal}

Para obter-se uma expressão geral que permita calcular de modo fácil os momentos das diversas ordens, obtém-se a f.g.m. para a distribuição multinormal, a partir de sua definição.

$$
m_{x_{1}}, \ldots, x_{n}\left(t_{1}, \ldots, t_{n}\right)=e^{(1 / 2)\left(T^{\prime} \sigma T\right)}
$$

pois,

$$
\begin{aligned}
m_{X_{1}} \ldots x_{n}\left(t_{1}, \ldots, t_{n}\right) & =E\left[e^{\left.x_{1} t_{1}+x_{2} t_{2}+\ldots+x_{n} t_{n}\right]}\right. \\
& =E\left[e^{\left.X^{\prime} T\right]}\right. \\
& =\int_{-\infty}^{+\infty} e^{X^{\prime} T} g_{X_{1}}, \ldots, x_{n}\left(x_{1}, \ldots, x_{n}\right) d X \\
& =\frac{\sqrt{|s|}}{(2 \pi)^{n / 2}} \int_{-\infty}^{+\infty} e^{X^{\prime} T-(b / 2)\left(X^{\prime} \sigma^{-1} X\right)} d X
\end{aligned}
$$

onde:

$$
\begin{aligned}
& T=\left[\begin{array}{l}
t_{1} \\
t_{2} \\
\cdots \\
t_{n}
\end{array}\right] \\
& d x=\left[\begin{array}{c}
d x_{1} \\
d x_{2} \\
\cdots \\
d x_{n}
\end{array}\right] \\
& g_{x_{1}}, \ldots, x_{n}\left(x_{1}, \ldots, x_{n}\right)=\frac{\sqrt{|s|}}{(2 \pi)^{n / 2}} e^{(-1 / 2)\left(x^{\prime} \sigma^{-1} x\right)}
\end{aligned}
$$


Para resolver a integral correspondente à função geradora de momentos, procede-se da seguinte maneira.

Seja

$$
\Omega(X)=-\frac{1}{2} X^{\prime} \sigma^{-1} X+X^{\prime} T
$$

e

$$
\begin{aligned}
d \Omega(x) & =-\frac{1}{2}(d x)^{\prime} \sigma^{-1} x-\frac{1}{2} x^{\prime} \sigma^{-1}(d x)+(d x)^{\prime} T \\
& =-(d x)^{\prime}\left[\sigma^{-1} x-T\right]
\end{aligned}
$$

Tomando-se $d \Omega(X) \equiv 0$, tem-se

$$
\begin{aligned}
\sigma^{-1} \mathrm{X}-\mathrm{T} & \equiv 0 \\
\sigma^{-1} \mathrm{X} & \equiv \mathrm{T} \\
\sigma \sigma^{-1} \mathrm{X} & \equiv \sigma \mathrm{T} \\
\mathrm{X}_{0} & \equiv \sigma \mathrm{T}
\end{aligned}
$$

e, substituindo-se

$$
\begin{aligned}
X & =X_{0}+Y \\
X & =\sigma T+Y \\
d X & =d Y,
\end{aligned}
$$

obtēm-se

$$
\begin{aligned}
\Omega(X) & =\Omega\left(X_{0}+Y\right)=\Omega(\sigma T+Y) \\
& =\Omega\left(X_{0}\right)-\frac{1}{2} Y^{\prime} \sigma^{-1} Y
\end{aligned}
$$

e, como $\sigma=\sigma^{\prime}$, 


$$
\begin{aligned}
\Omega(X) & =-\frac{1}{2}\left(T^{\prime} \sigma+Y^{\prime}\right) \sigma^{-1}(\sigma T+Y)+\left(T^{\prime} \sigma+Y^{\prime}\right) T \\
& =-\frac{1}{2}\left[T^{\prime} \sigma \sigma^{-1} \sigma T+T^{\prime} \sigma \sigma^{-1} Y+Y^{\prime} \sigma^{-1} \sigma T+Y^{\prime} \sigma^{-1} Y\right]+T^{\prime} \sigma T+Y^{\prime} T \\
& =\frac{1}{2} T^{\prime} \sigma T-\frac{1}{2} Y^{\prime} \sigma^{-1} Y
\end{aligned}
$$

e, substituindo-se esse resultado em (3), tem-se que

$$
\begin{aligned}
m_{X_{1}}, \ldots, X_{n}\left(t_{1}, \ldots, t_{n}\right) & =\frac{\sqrt{|S|}}{(2 \pi)^{n / 2}} \int_{-\infty}^{+\infty} e^{-(1 / 2) Y^{\prime} \sigma^{-1} Y+(1 / 2) T^{\prime} \sigma T} d Y \\
& =e^{(1 / 2) T^{\prime} \sigma T} \frac{\sqrt{|S|}}{(2 \pi)^{n / 2}} \int_{-\infty}^{+\infty} e^{-(1 / 2) Y^{\prime} \sigma^{-1} Y} d Y
\end{aligned}
$$

e, como

$$
\frac{\sqrt{|S|}}{(2 \pi)^{n / 2}} \int_{-\infty}^{+\infty} e^{-(1 / 2) Y^{\prime} \sigma^{-1} Y} d Y=1
$$

tem-se finalmente que

$$
m_{x_{1}}, \ldots, x_{n}\left(t_{1}, \ldots, t_{n}\right)=e^{(1 / 2) T^{\prime} \sigma T}
$$

é a função geradora de momentos para a distribuição multinormal.

A partir dessa expressão geral, é possível obter-se a f.g.m. para distribuições multinormais. Cita-se, a título de ilus traçäo, a distribuição binormal, e calculam-se os seus principais mo mentos. 


\subsection{1 - Função geradora de momentos} para a distribuição binormal

Sejam $x_{1} \cap N\left(0, \sigma_{1}^{2}\right)$ e $x_{2} \cap N\left(0, \sigma_{2}^{2}\right)$ variäveis aleatórias com distribuição binormal de probabilidade. A sua função geradora de momentos é dada por

$$
\begin{aligned}
& m_{x_{1}}, x_{2}\left(t_{1}, t_{2}\right)=e^{1 / 2\left[t_{1} t_{2}\right]\left[\begin{array}{cc}
\sigma_{1}^{2} & \rho \sigma_{1} \sigma_{2} \\
\rho \sigma_{1} \sigma_{2} & \sigma_{2}^{2}
\end{array}\right]\left[\begin{array}{l}
t_{1} \\
t_{2}
\end{array}\right]} \\
& =e^{1 / 2\left[t_{1}^{2} \sigma_{1}^{2}+2 \rho t_{1} t_{2} \sigma_{1} \sigma_{2}+t_{2}^{2} \sigma_{2}^{2}\right]}
\end{aligned}
$$

e seus principais momentos são

$$
E\left(X_{1}\right)=0 \quad \text { e } \quad E\left(X_{2}\right)=0
$$

pois,

$$
\begin{aligned}
E\left(X_{1}\right) & =\left.\frac{\partial}{\partial t_{1}} m_{X_{1}, X_{2}}\left(t_{1}, t_{2}\right)\right|_{t_{1}=t_{2}=0} \\
& =\left.\frac{1}{2}\left(2 t_{1}^{2} \sigma_{1}^{2}+2 \rho t_{2} \sigma_{1} \sigma_{2}\right) e^{1 / 2\left[t_{1} \sigma_{1}^{2}+2 \rho t_{1} t_{2} \sigma_{1} \sigma_{2}+t_{2}^{2} \sigma_{2}^{2}\right]}\right|_{t_{1}=t_{2}=0} \\
& =0 \quad E\left(X_{1}^{2}\right)=\sigma_{1}^{2} \quad \text { e } \quad E\left(X_{2}^{2}\right)=\sigma_{2}^{2}
\end{aligned}
$$

pois,

$$
E\left(X_{1}^{2}\right)=\frac{\partial^{2}}{\partial t_{1}^{2}} m_{X_{1}},\left.X_{2}\left(t_{1}, t_{2}\right)\right|_{t_{1}=t_{2}=0}
$$


79.

$$
\begin{array}{rlrl}
E\left(X_{1}^{2}\right)= & e^{1 / 2\left(t_{1}^{2} \sigma_{1}^{2}+2 \rho t_{1} t_{2} \sigma_{1} \sigma_{2}+t_{2}^{2} \sigma_{2}^{2}\right)}\left(t_{1} \sigma_{1}^{2}+\rho t_{2} \sigma_{1} \sigma_{2}\right)^{2}+ & \\
& +e^{1 / 2\left(t_{1}^{2} \sigma_{1}^{2}+2 \rho t_{1} t_{2} \sigma_{1} \sigma_{2}+t_{2}^{2} \sigma_{2}^{2}\right)} & \sigma_{1}^{2} & \mid t_{1}=t_{2}=0 \\
= & \sigma_{1}^{2} &
\end{array}
$$

$$
\begin{gathered}
\operatorname{Var}\left(\mathrm{X}_{1}\right)=\sigma_{1}^{2} \text { e } \operatorname{Var}\left(\mathrm{X}_{2}\right)=\sigma_{2}^{2} \\
\mathrm{E}\left(\mathrm{X}_{1}, \mathrm{X}_{2}\right)=\rho \sigma_{1} \sigma_{2}
\end{gathered}
$$

pois,

$$
\begin{aligned}
& E\left(X_{1}, X_{2}\right)=\frac{\partial^{2}}{\partial t_{1} \partial t_{2}} \mathbb{m}_{1},\left.x_{2}\left(t_{1}, t_{2}\right)\right|_{t_{1}=t_{2}=0} \\
& =e^{1 / 2\left(t_{1}^{2} \sigma_{1}^{2}+2 \rho t_{1} t_{2} \sigma_{1} \sigma_{2}+t_{2}^{2} \sigma_{2}^{2}\right)}\left(t_{1} \sigma_{1}^{2}+\rho t_{2} \sigma_{1} \sigma_{2}\right) \times \\
& \times\left(\rho t_{1} \sigma_{1} \sigma_{2}+t_{2} \sigma_{2}^{2}\right)+e^{1 / 2\left(t_{1}^{2} \sigma_{1}^{2}+2 \rho t_{1} t_{2} \sigma_{1} \sigma_{2}+t_{2}^{2} \sigma_{2}^{2}\right)} \times \\
& \times\left(\rho \sigma_{1} \sigma_{2}\right) \\
& =\rho \sigma_{1} \sigma_{2} \\
& \text { E conveniente observar-se que, se as variäveis alea- } \\
& \text { tōrias independentes têm distribuição normal, tal que } \\
& x_{1} \cap N\left(m_{1}, \sigma_{1}^{2}\right) \quad \text { e } \quad x_{2} \cap N\left(m_{2}, \sigma_{2}^{2}\right) \\
& -\infty<m_{i}<\infty \quad, \quad i=1,2
\end{aligned}
$$

a sua densidade de probabilidade é dada por 


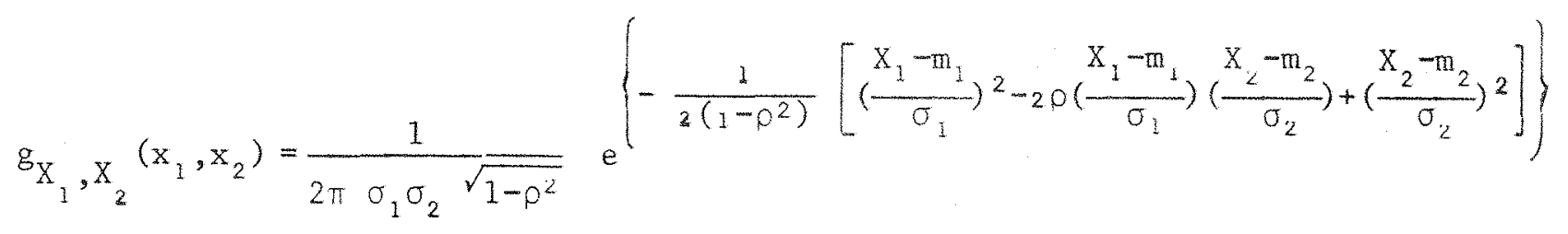

e a sua função geradora de momentos, por

$$
m_{X_{1}, x_{2}}\left(t_{1}, t_{2}\right)=e^{\left[t_{1} m_{1}+t_{2} m_{2}+(1 / 2)\left(t_{1}^{2} \sigma_{1}^{2}+2 \rho t_{1} t_{2} \sigma_{1} \sigma_{2}+t_{2}^{2} \sigma_{2}^{2}\right)\right]}
$$

de forma que, para o cálculo da f.g.m., basta que se adicione ao ex poente na forma matricial geral o vetor da combinação linear

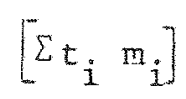

de forma que

$$
m_{x_{1}}, \ldots, x_{n}\left(t_{1}, \ldots, t_{n}\right)=e^{\left[\sum t_{i} m_{i}\right]+(i / 2) T^{\prime} \sigma T}
$$

3.5 - Förmula Geral para Obtenção dos Momentos nas Distribuições Bernoulli, Binomial, Poisson, Geométrica, Binomial Negativa, Normal, Gama e Exponencial (Negativa)

\subsection{1 - Fórmula recorrente (NOGUEIRA, 1965)}

Os momentos das distribuições Binomial, Poisson, Nor mal, Geométrica, Gama, Exponencial, Binomial Negativa e Bernoulli podem ser obtidos a partir de suas funções geradoras de momentos, co mo se desenvolve no sub-capítulo 3.4 deste trabalho. Porẻm, o cálcu lo dos momentos pelo mëtodo da função geradora, embora geral, não ë de fäcil aplicação quando se pretende determinar momentos de ordem 
superior à segunda. Para facilitar esse cālculo, apresenta-se a fór mula recorrente (NOGUEIRA, 1965)

$$
\mu_{n+1}=\sigma^{2} \frac{d}{d m}(\mu n)+n \sigma^{2} \mu_{n-1}
$$

que foi, pelo citado autor, aplicada às distribuições Binomial, Pois son e Normal.

$$
\text { NOGUEIRA (1965) afirma que a förmula (4) è válida pạ }
$$
ra toda função densidade de probabilidade $f_{X}(x)$ que satisfaça à för mula

$$
\frac{d}{d m}\left[f_{X}(x)\right]=\left(\frac{X-m}{\sigma^{2}}\right) f_{X}(x)
$$

onde $\mathrm{m}$ e $\sigma^{2}$ são, respectivamente, a mëdia e a variância da função $f_{X}(x)$, e o demonstra, tanto para o caso da função densidade ser de variável discreta como continua.

- Caso em que a função densidade $\hat{f}_{X}(x)$ ¿ de variāvel discreta

Neste caso, sendo $\mathrm{N}$ inteiro e positivo, tem-se

$$
\mu_{\mathrm{n}}=\sum_{0}^{N}(\mathrm{x}-\mathrm{m})^{\mathrm{n}} \mathrm{f}(\mathrm{x})
$$

Derivando em relação a ma , obtēm-se:

$$
\begin{aligned}
\frac{d}{d m}\left(u_{n}\right) & =n \sum_{0}^{N}(x-m)^{n-1}(-1) f(x)+\sum_{0}^{N}(x-m)^{n} \frac{d}{d m} f(x) \\
& =-n \sum_{0}^{N}(x-m)^{n-1} f(x)+\sum_{0}^{N}(x-m)^{n} \frac{d}{d m} f(x) .
\end{aligned}
$$


82.

$$
\begin{aligned}
\text { Se } \frac{d}{d m} f(x) \text { satisfaz à förmula (5), } & \begin{aligned}
\frac{d}{d m}\left(\mu_{n}\right) & =-n \mu_{n-1}+\frac{x-m}{\sigma^{2}} f(x) \sum_{0}^{N}(x-m)^{n} \\
& =-n \mu_{n-1}+\frac{1}{\sigma^{2}} \mu_{n+1}
\end{aligned}
\end{aligned}
$$

e

$$
\mu_{n+1}=\sigma^{2} \frac{d}{d m}\left(\mu_{n}\right)+n \sigma^{2} \mu_{n-1} .
$$

- Caso em que a função de densidade $f_{X}(x)$ è de variāvel continua Neste caso,

$$
\mu_{n}=\int_{-\infty}^{+\infty}(x-m)^{n} f(x) d x
$$

Derivando em relação a $\underline{m}$, obtëm-se:

$$
\frac{d}{d m}\left(\mu_{n}\right)=-n \int_{-\infty}^{+\infty}(x-m)^{n-1} f(x) d x+\int_{-\infty}^{+\infty}(x-m)^{n} \frac{d}{d m} f(x) d x
$$

se $\frac{d}{d m} f(x)$ satisfaz à förmula (5)

$$
\begin{aligned}
\frac{d}{d m}\left(\mu_{n}\right) & =-n \mu_{n-1}+\int_{-\infty}^{+\infty}(x-m)^{n}\left(\frac{x-m}{\sigma^{2}} f(x) d x\right. \\
& =-n \mu_{n-1}+\frac{1}{\sigma^{2}} \mu_{n+1}
\end{aligned}
$$

e

$$
\mu_{n+1}=\sigma^{2} \frac{d}{d m}\left(\mu_{n}\right)+n \sigma^{2} \mu_{n-1} .
$$


83.

No presente trabalho, aplicou-se a fórmula (5) a cada uma das citadas distribuições, e depois obteve-se a fórmula recorrente (4), demonstrando-se assim que, além das distribuições Binomial, Poisson e Normal apresentarem uma fórmula geral para a obtenção dos momentos, essa fórmula pode ser generalizada para as dis tribuições Geométrica, Gama, Exponencial (Negativa), Binomial Negativa e Bernoulli.

Apresenta-se, neste capitulo, esse desenvolvimento pa ra cada uma das mencionadas distribuiçôes.

\subsection{2 - Distribuição de Bernoull i}

Em uma distribuição de Bernoulli, tem-se que:

$$
\left\{\begin{array}{l}
f_{X}(x)=p^{x}(1-p)^{1-x}=p^{x} q^{1-x}, \text { para } x=0 \text { ou } x=1 \\
E(X)=m=p, \operatorname{Var}(X)=\sigma^{2}=p q
\end{array}\right.
$$

Verifica-se inicialmente a fórmula:

$$
\begin{aligned}
& \frac{d}{d m}[f(x)]=\left(\frac{x-m}{\sigma^{2}} f(x)\right. \\
\frac{d}{d m} f(x)= & x p^{x-1} q^{1-x}+p^{x}(1-x) q^{-x}(-1) \\
= & p^{x} q^{3-x}\left[\frac{x}{p}-\frac{(1-x)}{q}\right]
\end{aligned}
$$


84.

$$
\begin{aligned}
& \frac{d}{d m} f(x)=p^{x} q^{1-x}\left[\frac{x(p+q)-p}{p q}\right] \\
& =p^{x} q^{1-x} \underset{p q}{\left(\frac{x-p}{p}\right)} \\
& =f(x)\left(\frac{x-m}{\sigma^{2}}\right) \\
& \text { e a seguir obtēm-se a fórmula recorrente } \\
& \mu_{n+1}=\sigma^{2} \frac{d}{d m}\left(\mu_{n}\right)+n \sigma^{2} \mu_{n-1}, \\
& \text { pois o momento de ordem } n \vec{e} \text {, por definição, } \\
& \mu_{n}=\sum_{0}^{1}(x-p)^{n} p^{x} q^{1-x} \\
& \text { e, derivando-o em relaçāo a } p \text {, lembrando que } q=1 \text { - } p \text { e, portanto, } \\
& \frac{d q}{d p}=-1 \text {, obtem-se: } \\
& \frac{d}{d p}\left(\mu_{n}\right)=\Sigma\left[\begin{array}{l}
-n(x-p)^{n-1} p^{x} q^{1-x}+x(x-p)^{n} p^{x} p^{-1} q^{1-x}- \\
-(x-p)^{n} p^{x}(1-x) q^{-x} \frac{q}{q}
\end{array}\right] \\
& =-n \mu_{n-1}+\frac{x}{p} \mu_{n}-\frac{(1-x)}{q} \mu_{n} \\
& p q \frac{d}{d p}\left(\mu_{n}\right)=-n p q \mu_{n-1}+x q \mu_{n}-(1-x) p \mu_{n} \\
& =-n p q \mu_{n-1}+(x-p) \mu_{n}
\end{aligned}
$$




$$
p q \frac{d}{d p}\left(\mu_{n}\right)=-n p q \mu_{n-1}+\mu_{n+1}
$$

$$
\begin{aligned}
& \text { e, como } p q=\sigma^{2} \text { e } p=m, \\
& \mu_{n+1}=\sigma^{2} \frac{d}{d m}\left(\mu_{n}\right)+n \sigma^{2} \mu_{n-1}
\end{aligned}
$$

\subsection{3 - Distribuição Binomial}

Em uma distribuição binomial, tem-se que:

$$
\begin{aligned}
& \begin{cases}f_{X}(x)=\left(\begin{array}{l}
N \\
x
\end{array}\right) p^{x} q^{N-x} & , \quad \text { para } X=0,1,2, \ldots, N \\
\operatorname{com} 0 \leq p \leq 1, & q=1-p \\
m=E(X)=N p, & \sigma^{2}=\operatorname{Var}(X)=N p q\end{cases} \\
& \text { Verifica-se inicialmente a fórmula } \\
& \frac{d}{d m}[f(x)]=\left(\frac{x-m}{\sigma^{2}}\right) f(x) \\
& \frac{d}{d m}[f(x)]=\left(\begin{array}{l}
N \\
x
\end{array}\right)\left[x p^{x-1} \frac{1}{N} q^{N-x}+p^{x}(N-x) q^{N-x-1}\left(-\frac{1}{N}\right)\right] \\
& \frac{d}{d m}[f(x)]=\frac{1}{N}\left({ }_{x}^{N}\right)\left[\frac{x p^{x} q^{N-x}}{p}-\frac{p^{x} q^{N-x}(N-x)}{q}\right] \\
& =\frac{1}{N}\left(\begin{array}{l}
N \\
x
\end{array}\right)\left[\frac{x p^{x} q^{N-x} q-p^{x} q^{N-x}(N-x) p}{p q}\right] \\
& =\frac{1}{N}\left(\begin{array}{l}
N \\
x
\end{array}\right) p^{x} q^{N-x}\left[\frac{x q-p(N-x)}{p q}\right]
\end{aligned}
$$




$$
\begin{aligned}
\frac{d}{d m} f(x) & =\frac{1}{N}\left(\begin{array}{l}
N \\
x
\end{array}\right) p^{x} q^{N-x}\left[\frac{x(q+p)-N p}{p q}\right] \\
& =\left(\begin{array}{l}
N \\
x
\end{array}\right) p^{x} q^{N-x}\left[\frac{x-N p]}{N p q}\right] \\
& =f(x)\left(\frac{x-m}{\sigma^{2}}\right)
\end{aligned}
$$

e a seguir obtém-se a fórmula recorrente

$$
\mu_{n+1}=\sigma^{2} \frac{d}{d m}\left(\mu_{n}\right)+n \sigma^{2} \mu_{n-1}
$$

pois, o momento de ordem $n$ è, por definição,

$$
u_{n}=\sum_{0}^{N}(x-N p)^{n}\left({ }_{x}^{N}\right) p^{x} q^{N-x}
$$

e, derivando-o em relação a $p$, lembrando que $q=1-p$ e $\frac{d q}{d p}=-1$, obtēm-se:

$$
\begin{aligned}
\frac{d}{d p}\left(\mu_{n}\right) & =\Sigma\left[\begin{array}{l}
-n N(x-N p)^{n-1} f(x)+(x-N p)^{n}\left(\begin{array}{l}
N \\
x
\end{array}\right) \times p^{x-1} q^{N-x}- \\
-(N-x)(x-N p)^{n}\left(\begin{array}{l}
N \\
x
\end{array}\right) p^{x} q^{N-x-1}
\end{array}\right] \\
& =-N n \mu_{n-1}+\frac{x}{p} \mu_{n}-\frac{(N-x)}{q} \mu_{n} \\
& p q \frac{d}{d p}\left(\mu_{n}\right)=-n N p q \mu_{n-1}+x q \mu_{n}-(N-x) p \mu_{n} \\
& =-n N p q \mu_{n-1}+(x-N p) \mu_{n} \\
& =-n N p q \mu_{n-1}+\mu_{n+1}
\end{aligned}
$$




$$
\mu_{n+1}=p q \frac{d}{d p}\left(\mu_{n}\right)+n N p q \mu_{n-1}
$$

como

$$
m=N p \quad \frac{d p}{d m}=\frac{1}{N}
$$

e

$$
\frac{d}{d p}\left(\mu_{n}\right)=\frac{\frac{d}{d m}\left(\mu_{n}\right)}{\frac{d}{d m}(p)}=N \frac{d}{d m}\left(\mu_{n}\right)
$$

tem-se que

$$
\begin{aligned}
\mu_{n+1} & =N p q \frac{d}{d m}\left(\mu_{n}\right)+n N p q \mu_{n-1} \\
& =\sigma^{2} \frac{d}{d m}\left(\mu_{n}\right)+n \sigma^{2} \mu_{n-1}
\end{aligned}
$$

3.5.4 - Distribuição de Poisson

Em uma distribuição de Poisson, temme que:

$$
\left\{\begin{array}{l}
f_{X}(x)=\frac{e^{-\lambda} \lambda^{x}}{x !} \quad, \quad \text { para } x=0,1,2,3, \ldots \\
\operatorname{com} \lambda>0 \\
e m=E(X)=\lambda \\
\sigma^{2}=\operatorname{Var}(X)=\lambda
\end{array}\right.
$$

Verifica-se inicialmente a fórmula

$$
\frac{d}{d m}[f(x)]=\left(\frac{x-m}{\sigma^{2}}\right) f(x)
$$




$$
\begin{aligned}
\frac{d}{d m} f(x) & =\frac{1}{x !}\left[x \lambda^{x-1} e^{-\lambda}-\lambda^{x} e^{-\lambda}\right] \\
& =\frac{e^{-\lambda} \lambda^{x}}{x !}\left[\frac{x}{\lambda}-1\right] \\
& =\frac{e^{-\lambda} \lambda^{x}}{x !}\left[\frac{x-\lambda}{\lambda}\right] \\
\frac{d}{d m} f(x) & =f(x) \frac{(x-m}{\sigma^{2}}
\end{aligned}
$$

e a seguir obtëm-se a fórmula recorrente

$$
u_{n+1}=\sigma^{2} \frac{d}{d m}\left(\mu_{n}\right)+n \sigma^{2} \mu_{n-1}
$$

pois, o momento de ordem n é, por definição,

$$
\mu_{n}=\sum_{x=0}^{\infty}(x-\lambda)^{n} \frac{\lambda^{x} e^{-\lambda}}{x !}
$$

e, derivando-a em relação a $\lambda$, obtém-se:

$$
\begin{aligned}
\frac{d}{d \lambda}\left(\mu_{n}\right) & \left.=\sum_{x=0}^{\infty}\right]_{-(x-\lambda)^{n}} \frac{\lambda^{x}}{x !} e^{-\lambda} \\
& =-n \mu_{n-1}+x \lambda^{-1} \mu_{n}-\mu_{n} \\
x ! & \frac{\lambda^{x} e^{-\lambda}}{x !}+(x-\lambda)^{n} \frac{x \lambda^{x-1}}{-\lambda}- \\
& =-n \mu_{n-1}+\mu_{n}\left(\frac{x}{\lambda}-1\right) \\
& =-n \mu_{n-1}+\mu_{n}\left(\frac{x-\lambda}{\lambda}\right)
\end{aligned}
$$




$$
\begin{gathered}
\lambda \frac{d}{d \lambda}\left(\mu_{n}\right)=-n \lambda \mu_{n-1}+\mu_{n+1} \\
\mu_{n+1}=n \lambda \mu_{n-1}+\lambda \frac{d}{d \lambda}\left(\mu_{n}\right)
\end{gathered}
$$

$$
\begin{aligned}
& \text { como } m=\lambda \text { e } \sigma^{2}=\lambda, \\
& \mu_{n+1}=\sigma^{2} \frac{d}{d n d}\left(\mu_{n}\right)+n \sigma^{2} \mu_{n-1}
\end{aligned}
$$

3,5.5 - Distribuição Geomētrica (Pascal)

Em uma distribuição geomëtrica, tem-se que:

$$
\begin{cases}f_{X}(x)=p(1-p)^{x} & , \quad \text { para } x=0,1,2, \ldots \\ \operatorname{com} 0<p \leq 1, & q=1-p \\ m=E(X)=\frac{q}{p}, & \sigma^{2}=\operatorname{Var}(X)=\frac{q}{p^{2}}\end{cases}
$$

Verifica-se inicialmente a fórmula

$$
\begin{gathered}
\frac{d}{d m}[f(x)]=\left(\frac{x-m}{\sigma^{2}}\right) f(x) \\
\text { Observando-se que } \frac{d p}{d m}=-p^{2} \text { e } \frac{d q}{d m}=p^{2}, \\
\frac{d}{d m}[f(x)]=\frac{d}{d m}(p) q^{x}+p \times q^{x-1} \frac{d}{d m}(q)
\end{gathered}
$$




$$
\begin{aligned}
\frac{d}{d m}[f(x)] & =-p^{2} q^{x}+p x \frac{q^{x}}{q} p^{2} \\
& =p q^{x}\left[x \frac{p^{2}}{q}-p\right] \\
& =p q^{x}\left[\frac{p^{2}}{q}\left(x-\frac{q}{p}\right)\right] \\
\frac{d}{d m}[f(x)] & =f(x)\left(\frac{x-m}{\sigma^{2}}\right)
\end{aligned}
$$

e a seguir obtêm-se a förmula recorrente

$$
\mu_{n+1}=\sigma^{2} \frac{d}{d m}\left(\mu_{n}\right)+n \sigma^{2} \mu_{n-1}
$$

pois, o momento de ordem $\underline{n} \bar{e}$, por definição

$$
\mu_{n}=\Sigma\left(x-\frac{q}{p}\right)^{n} p q^{x}
$$

e, derivando-o em relação a p, obtēm-se:

$$
\begin{aligned}
& \frac{d}{d p}\left(\mu_{n}\right)=\Sigma\left[\begin{array}{rr}
n\left(x-\frac{q}{p}\right)^{n-1} & \frac{1}{p^{2}} p q^{x}+\left(x-\frac{q}{p}\right)^{n} q^{x} \frac{p}{p}- \\
& -\left(x-\frac{q}{p}\right)^{n} p \times q^{x-1}
\end{array}\right] \\
& =\frac{n}{p^{2}} \mu_{n-1}+\frac{1}{p} \mu_{n}-\frac{x}{q} \mu_{n} \\
& =\frac{n}{p^{2}} \mu_{n-1}-\frac{1}{q}\left(x-\frac{q}{p}\right) \mu_{n}
\end{aligned}
$$




$$
\begin{aligned}
& \frac{d}{d p}\left(\mu_{n}\right)=\frac{n}{p_{2}} \mu_{n-1}-\frac{1}{q} \mu_{n+1} \\
& q \frac{d}{d p}\left(\mu_{n}\right)=n \frac{q}{p^{2}} \mu_{n-1}-\mu_{n+1} \\
& \mu_{n+1}=n \frac{q}{p^{2}} \mu_{n-1}-q \frac{d}{d p}\left(\mu_{n}\right)
\end{aligned}
$$

\section{Como}

$$
\begin{aligned}
\frac{d}{d p}\left(\mu_{n}\right) & =\frac{\frac{d}{d m}\left(\mu_{n}\right)}{\frac{d}{d m}(p)}=-(m+1)^{2} \frac{d}{d m}\left(\mu_{n}\right) \\
\mu_{n+1} & =n \frac{q}{p^{2}} \mu_{n-3}+q(m+1)^{2} \frac{d}{d m}\left(\mu_{n}\right)
\end{aligned}
$$

e, como

$$
\begin{aligned}
& q(m+1)^{2}=q\left(\frac{q}{p}+1\right)^{2}=\frac{q}{p^{2}} \\
& \mu_{n+1}=n \frac{q}{p^{2}} \mu_{n-1}+\frac{q}{p^{2}} \frac{d}{d m}\left(\mu_{n}\right) \\
& \mu_{n+1}=\sigma^{2} \frac{d}{d m}\left(\mu_{n}\right)+n \sigma^{2} \mu_{n-1}
\end{aligned}
$$


92.

3.5.6 - Distribuição binomial negativa

En uma distribuição binomial negativa, tem-se que:

$$
\left\{\begin{array}{l}
f_{X}(x)=\left(\begin{array}{c}
-r \\
X
\end{array}\right) p^{r}(-q)^{x}=\left(\begin{array}{c}
r+x-1 \\
x
\end{array}\right) p^{r} q^{x}, \text { para } x=0,1,2, \ldots \\
\operatorname{com} r=1,2,3, \ldots ; 0<p \leq 1 ; \quad q=1-p \\
m=E(X)=\frac{r q}{p} ; \quad \sigma^{2}=\operatorname{Var}(X)=\frac{r q}{p^{2}}
\end{array}\right.
$$

Verifica-se inicialmente a fórmula

$$
\frac{d}{d m}[f(x)]=\left(\frac{x-m}{\sigma^{2}}\right) f(x)
$$

Observando-se que

$$
\begin{aligned}
\frac{d p}{d m}=-\frac{p^{2}}{r} \quad \text { e } \frac{d q}{d m}=\frac{p^{2}}{r} & \\
\frac{d}{d m} f(x) & =\left(\begin{array}{c}
r+x-1 \\
x
\end{array}\right)\left[-p^{2} p^{r-1} q^{x}+p^{r} x q^{x-1} \frac{p^{2}}{r}\right] \\
& =\left(\begin{array}{c}
r+x-1 \\
x
\end{array}\right) p^{r} q^{x}\left[-p+\frac{x p^{2}}{r q}\right] \\
& =\left(\begin{array}{c}
r+x-1 \\
x
\end{array}\right) p^{r} q^{x}\left[\frac{p^{2}}{r q}\left(x-\frac{r q}{p}\right)\right] \\
\frac{d}{d m} f(x) & =f(x) \stackrel{(x-m}{\sigma^{2}}
\end{aligned}
$$


E a seguir obtēm-se a fórmula recorrente

$$
\mu_{n+1}=\sigma^{2} \frac{d}{d m} \mu_{n}+n \sigma^{2} \mu_{n-1}
$$

pois, o momento de ordem $\underline{n} \bar{e}$, por definição,

$$
\mu_{n}=\Sigma\left(x-\frac{r q) n}{p}\left(\begin{array}{c}
r+x-1 \\
x
\end{array}\right) p^{r} q^{x}\right.
$$

e, derivando-o em relação a p, obtēn-se:

$$
\begin{aligned}
& \frac{d}{d p}\left(\mu_{n}\right)=\Sigma\left[\begin{array}{c}
n\left(x-\frac{r q}{p}\right)^{n-1} \frac{r}{p^{2}} p^{r} q^{x}+\left(x-\frac{r q}{p}\right)^{n} r p^{r-1} q^{x}- \\
p \\
\\
\end{array}\right. \\
& \frac{d}{d p}\left(\mu_{n}\right)=\Sigma\left(\begin{array}{c}
r+x-1 \\
x
\end{array}\right)\left[\begin{array}{cc}
\left(x-\frac{r q}{p}\right)^{n-1} & p^{r} q^{x} \frac{n r}{p^{2}}+\left(x-\frac{r q}{p}\right)^{n} p^{r} q^{x} \frac{r}{p}- \\
& -\left(x-\frac{r q}{p}\right)^{n} p^{r} q^{x} \frac{x}{q}
\end{array}\right] \\
& =\mu_{n-1} \frac{n r}{p^{2}}+\frac{r}{p} \mu_{n}-\frac{x}{q} \mu_{n} \\
& =n \frac{r}{p^{2}} \mu_{n-1}-\frac{1}{q}\left(x-\frac{r q}{p}\right) \mu_{n} \\
& =n \frac{r}{p^{2}} \mu_{n-1}-\frac{1}{q} \mu_{n+1}
\end{aligned}
$$




$$
\begin{aligned}
& q \frac{d}{d p}\left(\mu_{n}\right)=n \frac{r q}{p^{2}} \mu_{n-1}-\mu_{n+1} \\
& \mu_{n+1}=n \frac{r q}{p^{2}} \mu_{n-1}-q \frac{d}{d p}\left(\mu_{n}\right)
\end{aligned}
$$

e, como

$$
\begin{aligned}
& \frac{d}{d p}\left(\mu_{n}\right)=\frac{\frac{d}{d m}\left(\mu_{n}\right)}{\frac{d}{d m}(p)}=\frac{\frac{d}{d m}\left(\mu_{n}\right)}{-\frac{p^{2}}{r}}=\frac{-r}{p^{2}} \frac{d}{d m}\left(\mu_{n}\right) \\
& \mu_{n+1}=n \frac{r q}{p^{2}} \mu_{n-1}+\frac{r q}{p^{2}} \frac{d}{d m}\left(\mu_{n}\right)
\end{aligned}
$$

ou seja,

$$
\mu_{n+1}=\sigma^{2} \frac{d}{d m}\left(\mu_{n}\right)+n \sigma^{2} \mu_{n-1}
$$

\subsection{7 - Distribuiçao exponencial (negativa)}

Em uma distribuição exponencial (negativa), tem-se

$$
\left\{\begin{array}{c}
f_{X}(x)=\lambda e^{-\lambda x} \quad, \quad I_{\left.\Gamma_{0}, \infty\right)}(x) \\
\operatorname{com} \quad \lambda>0, \\
m=E(X)=\frac{1}{\lambda} \text { e } \sigma^{2}=\operatorname{Var}(X)=\frac{1}{\lambda^{2}}
\end{array}\right.
$$

Verifica-se inicialmente a förmula

$$
\frac{d}{d m}[f(x)]=\left(\frac{x-m}{\sigma^{2}}\right) f(x)
$$


observando-se que

$$
\begin{aligned}
\frac{d}{d m}(\lambda) & =-\frac{1}{m^{2}}=-\lambda^{2}, e \\
\frac{d}{d m}\left(e^{-\lambda x}\right) & =\frac{x}{m^{2}} e^{-\lambda x}=x \lambda^{2} e^{-\lambda x} \\
\frac{d}{d m} f(x) & =\frac{d}{d m}(\lambda) e^{-\lambda x}+\lambda \frac{d}{d m}\left(e^{-\lambda x}\right) \\
& =\lambda e^{-\lambda x}\left[-\lambda+x \lambda^{2}\right] \\
& =\lambda e^{-\lambda x}\left[\lambda^{2}\left(x-\frac{1}{\lambda}\right)\right] \\
\frac{d}{d m} f(x) & =f(x)\left(\frac{x-m}{\sigma^{2}}\right)
\end{aligned}
$$

e a seguir obtëm-se a förmula recorrente

$$
\mu_{n+1}=\sigma^{2} \frac{d}{d m}\left(\mu_{n}\right)+n \sigma^{2} \mu_{n-1}
$$

pois, o momento de ordem n é, por definição,

$$
\mu_{n}=\int_{0}^{\infty}(x-m)^{n} \lambda e^{-\lambda x} d x
$$

e, derivando-o em relação a m, obtëm-se:

$$
\begin{aligned}
\frac{d}{d m}\left(\mu_{n}\right) & =\int_{0}^{\infty}\left[\begin{array}{l}
\left.-n(x-m)^{n-1} \lambda e^{-\lambda x}-\lambda(x-m)^{n} \lambda e^{-\lambda x}+\right] \\
+x \lambda^{2}(x-m)^{n} \lambda e^{-\lambda x}
\end{array}\right] d x \\
& =-n \mu_{n-1}-\lambda \mu_{n}+x \lambda^{2} \mu_{n}
\end{aligned}
$$




$$
\begin{aligned}
\frac{d}{d m}\left(\mu_{n}\right) & =-n \mu_{n-1}+\lambda^{2}\left(x-\frac{1}{\lambda}\right) \mu_{n} \\
& =-n \mu_{n-1}+\lambda^{2} \mu_{n+1}
\end{aligned}
$$

de forma que

$$
\mu_{n+1}=\frac{1}{\lambda^{2}} \frac{d}{d m}\left(\mu_{n}\right)+\frac{1}{\lambda^{2}} n \mu_{n-1}
$$

ou seja,

$$
\mu_{n+1}=\sigma^{2} \frac{d}{d m}\left(\mu_{n}\right)+n \sigma^{2} \mu_{n-1}
$$

\subsection{8 - Distribuição gama}

Em uma distribuição gama tem-se

$$
\left\{\begin{array}{l}
\mathrm{E}_{\mathrm{X}}(\mathrm{x})=\frac{\lambda}{\Gamma(r)}(\lambda \mathrm{X})^{r-1} \mathrm{e}^{-\lambda \mathrm{x}}, \mathrm{I}_{[0, \infty)}(\mathrm{X}) \\
\mathrm{com} \mathrm{r}>0>0 \\
\mathrm{~m}=\mathrm{E}(\mathrm{X})=\frac{r}{\lambda}, \text { e } \sigma^{2}=\operatorname{Var}(\mathrm{X})=\frac{\mathrm{r}}{\lambda^{2}}
\end{array}\right.
$$

Verifica-se inicialmente a fórmula

$$
\frac{d}{d m} f(x)=\left(\frac{x-m}{\sigma^{2}}\right) f(x)
$$

observando-se que $\frac{d \lambda}{d m}=-\frac{\lambda^{2}}{r}$, 


$$
\begin{aligned}
& \frac{d}{d m} f(x)=\frac{1}{\Gamma(r)}\left[\begin{array}{l}
\left.\frac{d}{d m}(\lambda)(\lambda x)^{r-i} e^{-\lambda x}+\lambda(r-1)(\lambda x)^{r-2} \frac{d}{d m}(\lambda x)+\right] \\
+\lambda(\lambda x)^{r-1} e^{-\lambda x} \frac{d}{d m}(-\lambda x)
\end{array}\right] \\
& =\frac{1}{\Gamma(r)}\left[\begin{array}{l}
-\frac{\lambda}{r} \lambda(\lambda x)^{r-1} e^{-\lambda x} \cdots \\
-(r-1) \frac{\lambda^{2}}{r} \times \lambda(\lambda x)^{r-1}(\lambda x)^{-1} e^{-\lambda x}+ \\
+\frac{\lambda^{2}}{r} x \lambda(\lambda x)^{r-1} e^{-\lambda x}
\end{array}\right] \\
& =\frac{\lambda}{\Gamma(r)}(\lambda x)^{r-1} e^{-\lambda x}\left[-\frac{\lambda}{r}-(r-1) \frac{\lambda}{r}+x \frac{\lambda^{2}}{r}\right] \\
& =\frac{\lambda}{\Gamma(r)}(\lambda x)^{r-1} e^{-\lambda x}\left[\frac{\lambda^{2}}{r}\left(x-\frac{r}{\lambda}\right)\right]
\end{aligned}
$$

ou seja,

$$
\frac{d}{d m} f(x)=f(x) \underset{\sigma^{2}}{\left(\frac{x-m}{)}\right.}
$$

e a seguir obtém-se a fórmula recorrente

$$
\mu_{n+1}=\sigma^{2} \frac{d}{d m}\left(\mu_{n}\right)+n \sigma^{2} \mu_{n-1}
$$

pois, o momento de ordem $\underline{\text { n }} \vec{e}$, por definição,

$$
\mu_{n}=\int_{0}^{\infty}(x-m)^{n} \frac{\lambda}{\Gamma(r)}(\lambda x)^{r-1} e^{-\lambda x} d x
$$


e, derivando-o em relação a $m$, obtém-se:

$$
\begin{aligned}
& \frac{d}{d m}\left(\mu_{n}\right)=\left[\begin{array}{cc}
-\frac{n}{\Gamma(r)}(x-m)^{n-1} \lambda(\lambda x)^{r-1} e^{-\lambda x}- \\
-\frac{(x-m)^{n}}{\Gamma(r)} \frac{\lambda^{2}}{r}(\lambda x)^{r-1} e^{-\lambda x}- \\
-\frac{(x-m)^{n}}{\Gamma(r)} \lambda(r-1) \frac{(\lambda x)^{r-1}}{\lambda x} \frac{\lambda^{2}}{r} e^{-\lambda x}+ \\
+(x-m)^{n} \frac{\lambda}{\Gamma(x)}(\lambda x)^{r-1} e^{-\lambda x} \frac{\lambda^{2}}{r} x
\end{array}\right] d x \\
& =-n \mu_{n-1}-\frac{\lambda}{r} \mu_{n}-(r-1) \frac{\lambda}{r} \mu_{n}+\frac{\lambda^{2}}{r} x \mu_{n} \\
& =-n \mu_{n-1}+\mu_{n}\left(x-\frac{r}{\lambda}\right) \frac{\lambda^{2}}{r} \\
& =-n \mu_{n-1}+\frac{\lambda^{2}}{r} \mu_{n+1} \\
& \mu_{n+1}=\frac{r}{\lambda^{2}} \frac{d}{d m}\left(\mu_{n}\right)+n \frac{r}{\lambda^{2}} \mu_{n-1} \\
& \mu_{n+1}=\sigma^{2} \frac{d}{d m}\left(\mu_{n}\right)+n \sigma^{2} \mu_{n-1}
\end{aligned}
$$




\section{5 .9 - Distribuição normal}

Em uma distribuição normal, tem-se

$$
\left\{\begin{array}{l}
f_{X}(x)=\frac{1}{\sigma \sqrt{2 \pi}} e^{-\left[(x-m)^{2} i\left(2 \sigma^{2}\right)\right]},-\infty<x<\infty \\
\operatorname{com}-\infty<\pi<\infty \quad ; \quad \sigma>0 \\
m=E(X) \quad ; \quad \sigma^{2}=\operatorname{Var}(X)
\end{array}\right.
$$

Verifica-se inicialmente a förmula

$$
\begin{aligned}
& \frac{d}{d m} f(x)=\frac{\left(\frac{x-m}{\sigma^{2}}\right)}{\sigma^{2}(x)} \\
& \frac{d}{d m} f(x)=\frac{1}{\sigma \sqrt{2 \pi}} e^{-\left[(x-m)^{2} /\left(2 \sigma^{2}\right)\right]}\left(\frac{x-m}{\sigma^{2}}\right. \\
& \frac{d}{d m} f(x)=\left(\frac{x-m}{\sigma^{2}}\right) f(x)
\end{aligned}
$$

e a seguir obtëm-se a fórmula recorrente

$$
\mu_{n+1}=\sigma^{2} \frac{d}{d m}\left(\mu_{n}\right)+n \sigma^{2} \mu_{n-1}
$$

pois, o momento de ordem n é, por definição,

$$
\mu_{n}=\frac{1}{\sigma \sqrt{2 \pi}} \int_{-\infty}^{+\infty}(x-m)^{n} e^{-\left[(x-m)^{2} /\left(2 \sigma^{2}\right)\right]} d x
$$

e, derivando-o em relação a m, obtém-se: 


$$
\begin{aligned}
\frac{d}{d m}\left(\mu_{n}\right)= & -\frac{1}{\sigma \sqrt{2 \pi}} n \int_{-\infty}^{+\infty}(x-m)^{n-1} e^{-\left[(x-m)^{2} /\left(2 \sigma^{2}\right)\right]} d x- \\
& -\frac{1}{\sigma^{2}} \frac{1}{\sigma \sqrt{2 \pi}} \int_{-\infty}^{+\infty}(x-m)^{n+1} e^{-\left[(x-m)^{2} /\left(2 \sigma^{2}\right)\right]} \cdot d x \\
= & -n u_{n-1}+\frac{1}{\sigma^{2}} H_{n+1}
\end{aligned}
$$

ou seja,

$$
u_{n+1}=\sigma^{2} \frac{d}{d m}\left(\mu_{n}\right)+n \sigma^{2} \mu_{n-1}
$$

\section{6 - Técnica da Função Geradora de Momentos}

MOOD et alii (1974) apresentam o seguinte teorema: Se duas variäveis aleatórias, $X$ e $Y$, com funções de densidade $f_{X}(x)$ e $f_{Y}(x)$, possuem funções geradoras de momentos $m_{X}(t)$ e $m_{Y}(t)$, iguais para todo $\underline{t}$ no intervalo $-h<t<h, h>0$, elas têm funções de dis tribuição $F_{X}(x)$ e $F_{Y}(y)$ iguais (6). Isso corresponde a estabelecer que, se é possível obter a f.g.m. de uma variāvel aleatōria, teoricamente é possỉvel encontrar a distribuiçào da variável aleatória, desde que existe uma única função de distribuição para uma dada fun ção geradora de momentos.

Esse teorema (6), constitui-se na base do desenvolvi mento deste capítulo. 
101.

\subsection{1 - Descrição da técnica}

A técnica da função geradora de momentos é um mëtodo para determinação das funçøes de distribuição, construído sobre o conceito da função geradora de momentos, e na existência de uma üni ca função de distribuição para uma dada função geradora (conforme 6). Dadas as variáveis aleatōrias $x_{1}, x_{2}, \ldots, x_{n}$, com densidade $f_{x_{1}}, x_{2}, \ldots, x_{n}\left(x_{1}, x_{2}, \ldots, x_{n}\right)$ e funções $g_{1}\left(x_{1}, \ldots, x_{n}\right) \ldots$ $\ldots g_{K}\left(x_{1}, \ldots, x_{n}\right)$, obtém-se a distribuição conjunta de

$$
Y_{1}=g_{1}\left(X_{1}, \ldots, X_{n}\right) \ldots Y_{K}=g_{K}\left(X_{1}, \ldots, X_{n}\right)
$$

A função geradora de momentos conjuntos de $Y_{1}, Y_{2}, \ldots$ $\ldots, \mathrm{Y}_{\mathrm{K}}$, se existe, é dada por

$$
\begin{aligned}
m_{Y_{1}}, \ldots, Y_{K}\left(t_{1}, \ldots, t_{K}\right)= & E\left[e^{\left.t_{1} Y_{1}+t_{2} Y_{2}+\ldots+t_{K} Y_{K}\right]}\right. \\
= & \ldots \int e^{t_{1} g_{1}\left(X_{1}, \ldots, x_{n}\right)+\ldots+t_{K} g_{K}\left(x, \ldots, x_{n}\right)} \times \\
& \quad \times f_{X_{1}}, \ldots, x_{n}\left(x_{1}, \ldots, x_{n}\right) \prod_{i=1}^{n} d_{i}
\end{aligned}
$$

Se, apös efetuar essa integração, a função resultante de $t_{1}, t_{2}, \ldots, t_{K}$ pode ser reorganizada como uma $f . g . m$. conjuntos de varias distribuições conjuntas conhecidas, segue-se que $Y_{d}$, $\mathrm{Y}_{2}, \ldots, \mathrm{Y}_{\mathrm{K}}$ tem aquela distribuição conjunta, em virtude do estabe lecido, que uma f.g.m., quando existe, é ünica e unicamente determi nada por sua função de distribuição (conforme 6). 
Apesar da grande aplicação dessa técnica, para k > 1 ela é de utilização prätica restrita, pois poucas f.g.m. conjuntas são passiveis de reconhecimento imediato. Porēm, para $k=1$, tem-se melhores possibilidades de reconhecer a f.g.m. resultante, pois a mesma é função de um argumento único.

Essa técnica, em conexão com a teoria das transforma ções, torna-se um instrumento poderoso na determinação de distribui ções, se conhecidas as suas funções geradoras de momentos.

3.6.1.1 - Aplicações da tēcnica de funções geradoras de momentos à obtenção de distribuições conjuntas

\subsubsection{1 - obtenção da distribuição de $Y=X^{2}$, admitindo-se $X$ nor- malmente distribuida com më- dia zero e variāncia um}

Se $X \cap N(0,1)$, a sua densidade de probabilidade é da

da por

$$
\begin{gathered}
f_{X}(x)=\frac{1}{\sqrt{2 \pi}} e^{-x^{2} / 2},-\infty<x<+\infty \\
\text { Obtém-se a seguir a } f \cdot g \cdot m \text {. de } Y=X^{2} \\
\pi_{Y}(t)=E\left[e^{t Y}\right]=\frac{1}{\sqrt{2 \pi}} \int_{-\infty}^{+\infty} e^{t x^{2}} e^{-x^{2} / 2} d x \\
=\frac{1}{\sqrt{2 \pi}} \int_{-\infty}^{+\infty} e^{-(1 / 2) x^{2}(1-2 t)} d x
\end{gathered}
$$




$$
m_{Y}(t)=\frac{1}{\sqrt{2 \pi}} \frac{(1-2 t)^{-1 / 2}}{(1-2 t)^{-1 / 2}} \int_{-\infty}^{+\infty} e^{-(1 / 2) x^{2}(1-2 t)} d x
$$

e, substituindo-se

$$
\begin{aligned}
u^{2} & =\frac{x^{2}}{2}(1-2 t) \\
u & =\frac{x}{\sqrt{2}} \sqrt{1-2 t} \\
\frac{\sqrt{2}}{\sqrt{1-2 t}} d u & =d x
\end{aligned}
$$

tem-se que

$$
\begin{aligned}
\mathrm{nn}_{Y}(t) & =\frac{1}{\sqrt{2 \pi}} \frac{(1-2 t)^{-1 / 2}}{(1-2 t)^{-1 / 2}} \int_{-\infty}^{+\infty} e^{-u^{2}} \frac{\sqrt{2}}{\sqrt{1-2 t}} d u \\
& =\frac{1}{\sqrt{\pi}}(1-2 t)^{-1 / 2} 2 \int_{0}^{\infty} e^{-u^{2}} d u
\end{aligned}
$$

e, como

$$
2 \int_{0}^{\infty} e^{-u^{2}} d u=\Gamma(1 / 2)=\sqrt{\pi}
$$

segue-se que

$$
\mathrm{m}_{\mathrm{Y}}(t)=(1-2 t)^{-1 / 2}
$$

Conhecendo-se a função geradora de momentos para a distribuição gama, que é

$$
\mathrm{m}_{X}(t)=\left(\frac{\lambda}{\lambda-t}\right)^{r}=\left(\frac{\lambda-t}{\lambda}\right)^{-r}=\left(1-\frac{t}{\lambda}\right)^{-r},
$$


e admitindo-se $\lambda=\frac{1}{2} ; r=\frac{1}{2}$, verifica-se facilmente que

$$
m_{X}(t)=(1-2 t)^{-1 / 2} \text {, }
$$

o que torna evidente o fato de que $m_{Y}(t)=(1-2 t)^{-1 / 2}$ ë a função ge radora de momentos de uma V.A. com distribuição gama, $\operatorname{com} \lambda=\frac{1}{2} \mathrm{e}$ $r=\frac{1}{2}$, do que se conclui que a densidade de probabilidade de $Y=X^{2} \bar{e}$ dada por

$$
g_{Y}(y)=\frac{1}{2^{1 / 2} \Gamma(1 / 2)} e^{-y / 2} y^{-1 / 2}
$$

que é também a densidade de probabilidade de uma distribuição de qui-quadrado com 1 (um) grau de 1 iberdade.

$$
\begin{aligned}
& \text { Dessa forma, se } x \cap N(0,1) \text { e } Y=X^{2} \text {, tem-se que } \\
& \qquad Y \cap X_{(1)}^{2}
\end{aligned}
$$

3.6.1.1.2 - Obtenção da distribuição con junta de $Y_{1}$ e $Y_{2}$, com

$$
Y_{1}=g_{1}\left(X_{1}, X_{2}\right)=X_{1}+X_{2}
$$$$
y_{2}=g_{2}\left(X_{1}, X_{2}\right)=x_{2}-x_{1}
$$

e $X_{1}$ e $X_{2}$ variāveis normais padronizadas independentes

Obtém-se inicialmente a função geradora de momentos conjuntos de $Y_{1} \in Y_{2}$ :

$$
\begin{aligned}
m_{Y_{1}, Y_{2}}\left(t_{1}, t_{2}\right) & =E\left[e^{\left[t_{1} Y_{1}+t_{2} Y_{2}\right.}\right] \\
& =E\left[e^{\left.\left(X_{1}+X_{2}\right) t_{1}+\left(X_{2}-X_{1}\right) t_{2}\right]}\right.
\end{aligned}
$$




$$
\begin{aligned}
m_{1}, Y_{2}\left(t_{3}, t_{2}\right) & =E\left[e^{\left[X_{1}\left(t_{1}-t_{2}\right)\right]}\left[E_{2}\left(t_{1}+t_{2}\right)\right]\right. \\
& =m_{X_{1}}\left(t_{1}-t_{2}\right) m_{X_{2}}\left(t_{1}+t_{2}\right) \\
& =e^{\left(t_{1}-t_{2}\right)^{2 / 2} e^{\left(t_{1}+t_{2}\right)^{2} / 2}} \\
& =e^{2 t_{1}^{2} / 2} e^{2 t_{2}^{2} / 2} \\
& =e^{1 / 2\left[2 t_{1}^{2}+2 t_{2}^{2}\right]} \\
& =m_{Y}\left(t_{1}\right) m_{Y_{2}}\left(t_{2}\right)
\end{aligned}
$$

Observa-se então que a funçäo resultante é a f.g.m. conjuntos de uma distribuição binormal, com $\mathrm{Y}_{1} \cap \mathrm{N}(0,2)$ e $\mathrm{Y}_{2} \cap \mathrm{N}(0,2)$ sendo $Y_{1}$ e $Y_{2}$ independentes, de forma que a distribuição conjunta de $Y_{1}, Y_{2}$ é binormal, com densidade de probabilidade dada por

$$
g_{Y_{1}, Y_{2}}\left(y_{1}, y_{2}\right)=\frac{1}{4 \pi} e^{-1 / 2\left[\left(y_{1}^{2} / 2\right)+\left(y_{2}^{2} / 2\right)\right]}
$$

3.6.1.1.3 - Obtenção da distribuição con junta de $\bar{X}$ e $\bar{Y}$

Suponha-se $\left(X_{i}, Y_{i}\right), i=1,2, \ldots, n$ uma amostra aleatō ria de tamanho n proveniente de uma distribuição normal bivariada, com função densidade de probabilidade $\mathrm{f}_{\mathrm{X}, \mathrm{Y}}(\mathrm{x}, \mathrm{y})$ e parâmetros $\mathrm{m}_{\mathrm{j}}$, $m_{2}, \sigma_{1}^{2}, \sigma_{2}^{2}$ e $\rho$ 
106.

Sejam

$$
\bar{X}=\frac{\sum_{i=1}^{n} X_{i}}{n} \quad \text { e } \bar{Y}=\frac{\sum_{i=1}^{n} Y_{i}}{n}
$$

Admitindo-se que os pares $\left(X_{i}, Y_{i}\right)$ são mutuamente in dependentes, e têm todos a mesma densidade de probabilidade, obtém-se a f.g.m. conjuntos $\mathrm{m}_{\bar{X}}, \overline{\mathrm{Y}}\left(\mathrm{t}_{1}, \mathrm{t}_{2}\right)$.

$$
\mathrm{m}_{\bar{X}}, \bar{Y}\left(t_{1}, t_{2}\right)=\int_{-\infty}^{+\infty} \cdots \int_{-\infty}^{+\infty} e^{\left[\left(t_{1} \sum x_{i} / n\right)+\left(t_{2} \sum y_{i} / n\right)\right]} \Omega d_{x_{1}} \ldots d_{n}
$$

onde $\rho=f\left(x_{1}, y_{1}\right) f\left(x_{2}, y_{2}\right) \ldots f\left(x_{n}, y_{n}\right)$

$$
\begin{aligned}
& \mathrm{m}_{\overline{\mathrm{X}}, \overline{\mathrm{Y}}}\left(\mathrm{t}_{1}, \mathrm{t}_{2}\right)=\prod_{i=1}^{n}\left[\int_{-\infty}^{+\infty} \int_{-\infty}^{+\infty} e^{\left[\left(t_{1} x_{i} / n\right)+\left(t_{2} y_{i} / n\right)\right]} f\left(x_{i}, y_{i}\right) d x_{i} d y_{i}\right] \\
& m_{\bar{X}, \bar{Y}}\left(t_{1}, t_{2}\right)= \\
& \prod_{i=1}^{n} e^{\left(\frac{t_{1} m_{1}}{n}+\frac{t_{2} m_{2}}{n}+1 / 2\left[\sigma_{1}^{2}\left(\frac{t_{1}}{n}\right)^{2}+2 p \sigma_{1} \sigma_{2}\left(\frac{t_{1}}{n}\right)\left(\frac{t_{2}}{n}\right)+\sigma_{2}^{2}\left(\frac{t_{2}}{n}\right)^{2}\right]\right)}
\end{aligned}
$$

que é a f.g.m. de uma distribuição normal bivariada com mëdias $m_{1}$ e $m_{2}$ e variâncias $\frac{\sigma_{1}^{2}}{n}$ e $\frac{\sigma_{2}^{2}}{n}$ e coeficiente de correlação 0 . Consequentemente, $\bar{X}$ e $\bar{Y}$ tem essa distribuição conjunta binormal com densidade conjunta dada por

$$
\begin{aligned}
& -\frac{1}{2\left(1-\rho^{2}\right)}\left[\frac{\left(\bar{x}-m_{1}\right)^{2}}{\frac{\sigma_{1}^{2}}{n}}-\rho\left(\frac{\bar{x}-m_{1}}{\frac{\sigma_{1}}{\sqrt{n}}}\right)\left(\frac{\bar{x}-m_{2}}{\frac{\sigma_{2}}{\sqrt{n}}}\right)+\frac{\left(\bar{y}-m_{2}\right)^{2}}{\frac{\sigma_{2}^{2}}{n}}\right] \\
& g_{\bar{X}}, \bar{Y}(\bar{x}, \bar{y})=\frac{n}{2 \pi \sigma_{1} \sigma_{2} \sqrt{1-\rho^{2}}} e
\end{aligned}
$$




\subsection{2 - Distribuiçao da soma de variāveis aleatörias inde- pendentes}

Mostra-se, neste sub-ítem, como utilizar a téenica da função geradora de momentos para se obter a distribuição da soma de V.A. independentes, através da utilização do teorema que se enuncia e demonstra a seguir.

$$
\begin{aligned}
& \text { Teorema: } \operatorname{se} x_{1}, x_{2}, \ldots, x_{n} \text { são V.A. independentes, e a f.g.m. } \\
& \text { de cada uma delas existe para todo } h>0 \text {, com }-\mathrm{h}<t< \\
& <\mathrm{h} \text {, e } \mathrm{Y}=\sum_{\mathrm{i}}^{\mathrm{n}} \mathrm{X}_{\mathrm{i}} \text {, então } \\
& m_{Y}(t)=E\left[e^{\Sigma X_{i} t^{\top}}\right]=\prod_{i=1}^{n} m_{X_{i}}(t) \quad \text {, para }-h<t<h
\end{aligned}
$$

Demonstraçăo :

$$
m_{Y}(t)=E\left[e^{\sum X_{i} t}\right]=E\left[\prod_{i=1}^{n} e^{X_{i} t}\right]=\prod_{i=1}^{n} E\left[e^{X_{i} t}\right]=\prod_{i=1}^{n} m_{i}(t)
$$

A grande utilidade prática desse teorema decorre do teorema que se apresenta no primeiro parágrafo deste capítulo,(6), que diz que uma f.g.m., quando existe, determina a função de distri buição. Dessa forma, se é possivel reconhecer $\prod_{i=1}^{n} m_{i}(t)$ como a f.g.m. correspondente a uma distribuição particular, então encontra-se a distribuição da soma das n variāveis aleatörias independentes $x_{i}$ 
3.6.2.1 - Apl icaçòes da técnica da f.g.m. à distrí buição da soma de V.A. independentes

\subsubsection{1 - obtenção da distribuição bi- nomial como soma de $n$ variá- veis aleatōrias independen- tes com distribuiçào de Ber- noulli}

Suponha-se que $x_{1}, x_{2}, \ldots, x_{n}$ são variäveis aleatórias de Bernoulli independentes, isto é, que

$$
P\left(X_{i}=1\right)=p \quad \text { e } \quad P\left(X_{i}=0\right)=1-p
$$

$\operatorname{com} \cdot \mathrm{m}_{\mathrm{X}_{i}}(\mathrm{t})=\mathrm{q}+\mathrm{pe} \mathrm{t}^{\mathrm{t}} \cdot$

Se $Y=\sum_{1}^{n} X_{i}$, a função geradora de $Y$, de acordo com o teorema apresentado em (7), é dada por

$$
m_{Y}(t)=m_{\Sigma X_{i}}(t)=\prod_{i=1}^{n} m_{i}(t)=\left(q+p e^{t}\right)^{n}
$$

que é a f.g.m. de uma V.A. binomial. Consequentemente, $Y=\sum_{1}^{n} X_{i}$ tem distribuição binomial com parâmetros

$$
\begin{aligned}
E(y) & =n p \\
\operatorname{Var}(y) & =n p q
\end{aligned}
$$

e densidade

$$
f_{Y}(y)=\left(\begin{array}{l}
n \\
y
\end{array}\right) p^{y} q^{n-y}
$$




\subsubsection{2 - Obtençăo da distribuição da soma de $n$ V.A. independentes com distribuiçăo de Poisson}

Suponha-se que $x_{1}, x_{2}, \ldots, x_{n}$ sào V.A. independentes com distribuição de Poisson, com

$$
E\left(X_{i}\right)=\lambda_{i} \quad \text { e } \quad m_{X_{i}}(t)=e^{\lambda_{i}\left(e^{t}-1\right)}
$$

Se $Y=\sum_{i=1}^{n} X_{i}$

$$
\begin{aligned}
m_{Y}(t) & =m_{\sum X_{i}}(t)=\prod_{i=1}^{n} m_{X_{i}}(t) \\
& =\prod_{i=1}^{n} e^{\lambda_{i}\left(e^{t}-1\right)}=e^{\sum \lambda_{i}\left(e^{t}-1\right)}
\end{aligned}
$$

que é novamente a f.g.m. de uma distribuição de V.A. Poisson com mé dia $\sum \lambda_{i}$. Consequentemente, $Y=\sum X_{i}$ tem distribuição Poisson com média

$$
E(Y)=\Sigma \lambda_{i}
$$

e densidade

$$
f_{Y}(y)=\frac{e^{-\sum \lambda_{i}}\left(\sum \lambda_{i}\right)^{y}}{y !} \quad, \quad y=0,1,2, \ldots
$$




\subsubsection{3 - Obtenção da distribuição ga- ma como soma de $\underline{n}$ V.A. inde- pendentes com distribuição exponencial (negativa)}

Suponha-se que $x_{1}, x_{2}, \ldots, x_{n}$ são V.A. independentes com distribuição exponencial (negativa), tal que

$$
\begin{gathered}
m_{X_{i}}(t)=\frac{\lambda}{\lambda-t} \quad, \quad t<\lambda \\
\text { Se } Y=\sum_{i=1}^{n} X_{i}, \\
m_{Y}(t)=m_{\Sigma X_{i}}(t)=\prod_{i=1}^{n} m_{X}(t)=\left(\frac{\lambda}{\lambda-t}\right)^{n}
\end{gathered}
$$

que é a função geradora de momentos de uma distribuição gama, com mē dia

$$
\mathrm{E}(\mathrm{Y})=\frac{\mathrm{n}}{\lambda}
$$

e densidade

$$
\begin{gathered}
\mathrm{f}_{Y}(\mathrm{y})=\frac{\lambda}{\Gamma(\mathrm{n})}(\lambda \mathrm{y})^{\mathrm{n}-\boldsymbol{I}} \mathrm{e}^{-\lambda \mathrm{y}}, \mathrm{I}_{[0, \infty)}(\mathrm{y}) \\
\mathrm{n}>0 \text { e } \lambda>0
\end{gathered}
$$




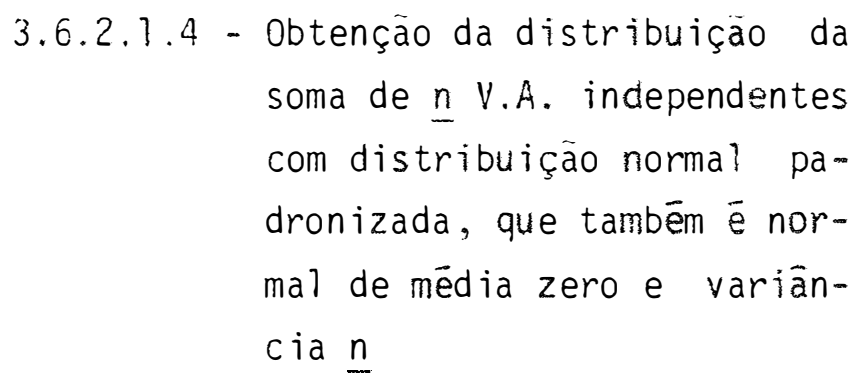

Suponha-se que $x_{1}, x_{2}, \ldots, x_{n}$ são V.A. independentes com distribuição normal padrão, tal que

$$
\begin{gathered}
m_{X_{i}}(t)=e^{(1 / 2) t^{2}} \\
\text { Se } Y=\sum_{i}^{n} X_{i}, \\
m_{Y}(t)=m_{\Sigma X_{i}}(t)=\prod_{i=1}^{n} m_{X_{i}}(t)=\left[e^{(1 / 2) t^{2}}\right]^{n}=e^{t^{2} n / 2}
\end{gathered}
$$

que é a f.g.m. de uma V.A. com distribuição normal de média zero e variância n. Consequentemente $Y$ tem distribuição normal, com densidade

$$
f_{Y}(y)=\frac{1}{\sqrt{n} \sqrt{2 \pi}} e^{-y^{2} /(2 n)}
$$




\subsubsection{5 - Obtenção da distribuição amos tral das médias de amostras aleatōrias de tamanho $\underline{n}$ a par tir da distribuição da soma de $\underline{n}$ V.A. independentes com distribuição normal}

Suponha-se que $x_{1}, x_{2}, \ldots, x_{n}$ são variāveis aleatō rias independentes com distribuição normal de média m e variância $\sigma^{2}$ tal que

$$
\begin{aligned}
m_{S}(t) & =m_{\sum X_{i}}(t)=\prod_{i=1}^{n} m_{X_{i}}(t) \\
& =\left[e^{\left.m t+\left(\sigma^{2} t^{2} / 2\right)\right]^{n}}\right. \\
& =e^{n m t+\left(n \sigma^{2} t^{2} / 2\right)}
\end{aligned}
$$

que é a f.g.m. de uma V.A. com distribuição normal, de média nm e variância $\underline{n}^{2}$, de forma que

$$
f_{S}(s)=\frac{1}{\sigma \sqrt{n} \sqrt{2 \pi}} e^{-(s-n m)^{2} /\left(2 n \sigma^{2}\right)}
$$

é a densidade de $S=\Sigma X_{i}$. 
Como

$$
\bar{X}=\frac{\Sigma X_{i}}{n}=\frac{S}{n},
$$

efetuando-se a transformação de variável, com $S=n \bar{x}$, e obtendo-se o Jacobiano da transformação

$$
|J|=\frac{d S}{d \bar{X}}=n
$$

resulta a densidade de probabilidade de $\bar{x}$

$$
f_{\bar{X}}(\bar{x})=\frac{1}{\frac{\sigma}{\sqrt{n}} \sqrt{2 \pi}} e^{-(\bar{x}-m)^{2} /\left(2 \sigma^{2} / n\right)}
$$

que é a densidade de probabilidade de uma V.A. com distribuição nor mal de média m e variância $\frac{\sigma^{2}}{n}$, de forma que se pode afirmar que, se $x \cap N\left(m, \sigma^{2}\right)$, também $\bar{x} \cap N\left(m, \frac{\sigma^{2}}{n}\right)$, ou seja, a média amostral $\bar{x}$, proveniente de uma população normal com média m e variância $\sigma^{2}$, tam bēm tem distribuição normal de média m e variância $\frac{\sigma^{2}}{n}$.

$$
\begin{aligned}
3.6 .2 .3 .6 \text { - } & \text { Obtenção da distribuição de } \\
& \text { qui-quadrado com }\left(r_{1}+r_{2}+\ldots+r_{n}\right) \\
& \text { graus de liberdade a partir } \\
& \text { da soma de } \underline{n} V . A \text {. independen } \\
& \text { tes com distribuição de qui- } \\
& \text {-quadrado com } r_{i} \text { graus de } 1 \underline{i} \\
& \text { berdade, } i=1,2, \ldots, n
\end{aligned}
$$

Suponha-se que $x_{1}, x_{2}, \ldots, x_{n}$ são variäveis aleatōrias independentes tendo distribuição de qui-quadrado com $r_{i}$ graus de 1 iberdade, 


$$
\begin{gathered}
x_{i} \cap x_{\left(r_{1}\right)}^{2} \\
x_{2} \cap x_{\left(r_{2}\right)}^{2} \\
\cdots \\
x_{n} \cap x_{\left(r_{n}\right)}^{2}
\end{gathered}
$$

tal que

$$
\begin{aligned}
\text { Se } Y=\sum_{i=1}^{n} x_{i}, & \\
m_{Y}(t) & =m_{\sum X_{i}}(t)=\prod_{i=1}^{n} m_{i}(t) \\
& =\prod_{i=1}^{n}(1-2 t)^{-r_{i} / 2} \\
m_{Y}(t) & =(1-2 t)^{-r_{i} / 2}
\end{aligned}
$$

que é a f.g.m. de uma V.A. com distribuiçäo de qui-quadrado com $\sum r_{i}$ graus de 1 iberdade. Consequentemente, $Y=\Sigma X_{i}$ tem distribuição $\chi_{\left(\sum r_{i}\right)}^{2}$ com densidade

$$
f_{Y}(y)=\frac{1}{2\left(\sum r_{i}\right) / 2} \frac{\Gamma\left(\sum r_{i} / 2\right)}{2 y / 2} y^{\left(\sum r_{i}-2\right) / 2}
$$


3.6.2.1.7 - Obtenção da distribuição binomial negativa a partir da soma de $\underline{n}$ V.A. independentes com distribuição geométrica

Suponha-se que $x_{1}, x_{2}, \ldots, x_{n}$ são variäveis aleatōrias independentes, cada qual com distribuição geométrica com parâme tro $\underline{p}$, tal que

$$
\begin{gathered}
m_{X_{i}}(t)=\frac{p}{1-q e^{t}} \\
\text { Se } Y=\sum_{i=1}^{n} X_{i}, \\
m_{Y}(t)=m_{\Sigma X_{i}}(t)=\prod_{i=1}^{n} m_{X_{i}}(t)=\prod_{i=1}^{n}\left(\frac{p}{1-q e^{t}}\right)=\left(\frac{p}{1-q e^{t}}\right)^{n}
\end{gathered}
$$

que é a f.g.m. de uma V.A. com distribuição binomial negativa de mé dia $E(X)=\frac{n q}{p}$.

$$
\text { Consequentemente, } Y=\Sigma X_{i} \text { tem distribuição binomial }
$$

negativa com densidade

$$
f_{Y}(y)=\left(\begin{array}{c}
n+y-1 \\
y
\end{array}\right) p^{n} q^{y}=\left(\begin{array}{c}
-n \\
y
\end{array}\right) p^{n}(-q)^{y},
$$

para $y=0,1,2, \ldots$ 
3.6.2.1.8 - obtenção da distribuição de $Y=\sum_{i=1}^{n} k_{i} X_{i}$

com $k_{i}$ constantes reais e $x_{i} \cap N\left(m_{i}, \sigma_{i}^{2}\right)$

Suponha-se que $x_{1}, x_{2}, \ldots, x_{n}$ são V.A. independentes distribuidas normalmente, tal que

$$
x_{i} \cap \mathrm{N}\left(\mathrm{m}_{\mathrm{i}}, \sigma_{\mathrm{i}}^{2}\right) \quad, \quad \mathrm{i}=1,2, \ldots, \mathrm{n}
$$

$k_{1}, k_{2}, \ldots, k_{n}$ constantes reais, e funções geradora de momentos,

$$
m_{k_{i} x_{i}}(t)=e^{k_{i} \cdot m_{i} t+\left(l^{1} / 2\right) k_{i}^{2} \sigma_{i}^{2} \cdot t^{2}}
$$

Se $Y=\sum_{i=1}^{n} k_{i} X_{i}$,

$$
\begin{aligned}
m_{Y}(t) & =m_{\sum k_{i} x_{i}}(t)=\prod_{i=1}^{n} m_{k_{i} x_{i}}(t) \\
& =\prod_{i=1}^{n}\left[e^{\left.k_{i} m_{i} t+(1 / 2) k_{i}^{2} \sigma_{i}^{2} t^{2}\right]}\right. \\
& =e^{\left(\sum_{i=1}^{n} k_{i} m_{i}\right) t+\left[\left(\sum_{i=1}^{n} k_{i}^{2} \sigma_{i}^{2}\right) t^{2}\right] / 2}
\end{aligned}
$$

que é a f.g.m. de uma V.A. com distribuição normal de média $\left(\sum_{i=1}^{n} k_{i} m_{i}\right)$ e variância $\left(\sum_{i=1}^{n} k_{i}^{2} \sigma_{l}^{2}\right)$. Consequentemente, $Y=\sum k_{i} X_{i}$ tem distribuição normal com média $\left(\sum k_{i} m_{i}\right)$ e variância $\left(\sum k_{i}^{2} \sigma_{i}^{2}\right)$ e densidade

$$
f_{Y}(y)=\frac{1}{\sqrt{2 \pi} \sqrt{\Sigma k_{i}^{2} \sigma_{i}^{2}}} e^{-\left(y-\sum k_{i} m_{i}\right)^{2} /\left[2\left(\sum k_{i}^{2} \sigma_{i}^{2}\right)\right]}
$$


Nota (4): 0 resultado obtido, $Y=\sum k_{i} X_{i} \cap N\left(\sum k_{i} m_{i} ; \sum k_{i}^{2} \sigma_{i}^{2}\right)$ corresponde a afirmar que a combinação linear de V.A. normais é também uma V.A. distribuỉda normalmente.

Em particular, se

$$
x_{1} \cap N\left(m_{1}, \sigma_{1}^{2}\right) \quad \text { e } \quad x_{2} \cap N\left(m_{2}, \sigma_{2}^{2}\right)
$$

sendo $X_{1}$ e $X_{2}$ independentes, então

$$
\begin{gathered}
\left(\mathrm{x}_{1}+\mathrm{x}_{2}\right) \cap \mathrm{N}\left(\mathrm{m}_{1}+\mathrm{m}_{2} ; \sigma_{1}^{2}+\sigma_{2}^{2}\right) \\
\mathrm{e} \\
\left(\mathrm{x}_{1}-\mathrm{X}_{2}\right) \cap \mathrm{N}\left(\mathrm{m}_{1}-\mathrm{m}_{2} ; \sigma_{1}^{2}+\sigma_{2}^{2}\right)
\end{gathered}
$$

3.6.2.1.9 - Obtenção da distribuição de

$$
y=\sum_{i=1}^{n}\left(\frac{x_{i}-m}{\sigma}\right)^{2}
$$

Suponharse que $x_{1}, x_{2}, \ldots, x_{n}$ são V.A. independentes distribuídas normalmente, com média m e variância $\sigma^{2}$,

$$
x_{i} \cap N\left(m, \sigma^{2}\right) \quad, \quad i=1,2, \ldots, n
$$

Como $\left(\frac{X_{i}-m}{\sigma}\right)$ tem distribuição normal padrão, tem-se que $\left(\frac{X_{i}-m}{\sigma}\right)^{2}$ tem distribuiçäo de qui-quadrado, com um grau de liberdade, de forma que

$$
\begin{aligned}
& \mathrm{m}_{\left(\frac{X^{-m}}{\sigma}\right)^{2}}(t)=(1-2 t)^{-1 / 2} \\
& \text { Se } Y=\sum_{i=1}^{n}\left(\frac{X_{i}-m}{\sigma}\right)^{2}, \text { então }
\end{aligned}
$$




$$
\begin{aligned}
\mathrm{m}_{Y}(t) & =\operatorname{II}_{i=1}^{\mathrm{n}} \mathrm{m}_{\left(\frac{x_{i}-\mathrm{m}}{\sigma}\right)^{2}}(t) \\
& =\left[(1-2 t)^{1 / 2}\right]^{\mathrm{n}} \\
& =(1-2 t)^{\mathrm{n} / 2}
\end{aligned}
$$

que é a f.g.m. de uma V.A. com distribuição de qui-quadrado com $\underline{n}$ graus de liberdade.

Consequent emente, $Y=\sum\left(\frac{X_{i}-m}{\sigma}\right)^{2}$ tem distribuiçáo de qui-quadrado com $\underline{n}$ graus de liberdade, ou seja:

$$
\mathrm{Y}=\sum\left(\frac{\mathrm{X}_{\mathrm{i}}-\mathrm{m}}{\sigma}\right)^{2} \cap \mathrm{X}_{(\mathrm{n})}^{2}
$$

3.6.3 - Aplicaçao da técnica da função geradora de momentos à obtenção de distribuições 1 imites

\subsubsection{1 - Função de distribuição limite}

Antes de se abordar como uma f.g.m. pode ser usada em problemas de distribuiçôes limites, uma definição básida deve ser apresentada.

Definição (1): (definição de função de distribuição limite) (HOOG e CRAIG, 1970)

Seja uma função de distribuição $F_{n}(y)$, de variävel aleatória $Y_{n}$, dependente de $\underline{n}$, uma constante positiva. Se $F(y)$ é uma função de distribuição, e se $\lim _{n \rightarrow \infty} F_{n}(y)=F(y)$ para todo y para o 
qual $F(y)$ é continua, então a variável aleatöria $Y_{n}$ e dita tendo uma distribuição limite, com função de distribuição limite $F(y)$.

Para obter a função de distribuição limite de uma va riāvel aleatória $Y_{n}$, pela utilização da definição (1), requer-se o conhecimento de $F_{n}(y)$ para cada constante positiva $\underline{n}$. Se essa funçào existe, a sua correspondente f.g.m., designada por $\mathbb{R}_{Y}(t, n)$, for nece um método conveniente para a determinação da função de distribuição limite, baseado no teorema que se segue.

Teorema: (HOOG e CRAIG, 1970)

Seja uma variāvel aleatōria $Y_{n}$, tendo uma função de distribuição $F_{n}(y)$ e função geradora de momentos $\mathbb{M}_{Y}(t ; n)$, que existe para $-h<t<h$, e para todo $\underline{n}$. Se existe uma função de distribuição $F(y)$, com correspondente função geradora de momentos $\mathbb{m}_{Y}(t)$, definida para $|t| \leq h_{1}<h$, tal que $\lim _{n \rightarrow \infty} m_{Y}(t, n)=m_{y}(t)$, então $Y_{n}$ tem uma distribuição limite, com função de distribuição $F(y)$.

Esse teorema habilita a afirmar que, sempre que uma V.A. tem una distribuição limite, se desejado, pode-se usar essa dis tribuição limite como uma aproximação para a exata função de distri buição, como mostra-se através de algumas aplicações. 
3.6.3.2 - Aplicação (1): Obtenção da distribuição limite da distribuição binomial quando $\underline{n}$ ë grande e $\underline{p}$ é pequeno

Seja $Y_{n}$ tendo uma distribuição que é binomial b(n,p). Suponha-se que a média $m=n p$ é a mesma para todo $\underline{n}$, isto é, $p=\frac{m}{n}-$, onde 뜨 é uma constante. Obtém-se a distribuição limite da distribui ção binomial, quando $\mathrm{p}=\frac{\mathrm{m}}{\mathrm{n}}$, através do limite de $\mathrm{m}_{\mathrm{Y}}(t ; n)$.

\section{Então, sendo}

$$
\begin{aligned}
\mathrm{m}_{\mathrm{Y}}(\mathrm{t} ; \mathrm{n}) & =\mathrm{E}\left[\mathrm{e}^{\mathrm{tY}} \mathrm{n}\right]=\left[1-p+p e^{t}\right]^{\mathrm{n}} \\
& =\left[1-\frac{\mathrm{m}}{\mathrm{n}}+\frac{\mathrm{m}}{\mathrm{n}} \mathrm{p} \mathrm{e}^{\mathrm{t}}\right]^{\mathrm{n}} \\
& =\left[1+\frac{\mathrm{m}\left(\mathrm{e}^{t}-1\right)}{\mathrm{n}}\right]^{\mathrm{n}}
\end{aligned}
$$

para todo t real.

E aplicando-se a seguir o limite a $m_{Y}(t ; n)$ para $\underline{n}$ ten dendo a infinito, pelo limite fundamental (1) do Apêndice, obtém-se

$$
\lim _{n \rightarrow \infty} m_{Y}(t ; n)=e^{m\left(e^{t}-1\right)}
$$

para todo $t$ real.

$$
\text { Lembrando que uma V.A. com distribuição de Poisson }
$$

tem f.g.m. dada por

$$
m_{X}(t)=e^{\lambda\left(e^{t}-1\right)},
$$


observa-se que a distribuição limite obtida é a f.g.m. de uma V.A. com distribuição de Poisson com $m=\lambda$.

Então, de acordo com o teorema citado (HOOG e CRAIG, 1970), e sob as condições estabelecidas, conclui-se que $Y_{n}$ tem uma distribuição limite Poisson com média m.

Dessa forma, mostra-se ser possivel usar a distribui ção de Poisson como uma aproximação para a distribuição binomial quando $\underline{n}$ é grande e $\mathrm{p}$ è pequeno.

\subsubsection{3 - Apl icação (2): Qbtenção da distribuição 1 i mite para $Y_{n}=\frac{Z_{n}-n}{\sqrt{2 n}}$ quando $\underline{n}$ é grande}

Seja $Z_{n}$ uma V.A. com distribuição de qui-quadrado com n graus de liberdade e função geradora de momentos,

$$
m_{Z_{n}}(t)=(1-2 t)^{-n / 2}, \quad t<\frac{1}{2} .
$$

A média e a variância de $Z_{n}$ são, respectivamente, $\underline{n}$ e $2 n$. Obtém-se a seguir a distribuição 1imite da V.A. $Y_{n}=\frac{Z_{n}-n}{\sqrt{2 n}}$. Para tanto, calcula-se a f.g.m. de $Y_{n}$, e a seguir aplica-se o limite para $n \rightarrow \infty$.

$$
\begin{aligned}
m_{Y}(t ; n)=E\left[e^{t Y_{n}}\right] & =E\left[e^{\left[t\left[\left(Z_{n}-n\right) / \sqrt{2 n}\right]\right.}\right] \\
& =e^{-t n / \sqrt{2 n}} E\left[e^{\left(t Z_{n}\right) / \sqrt{2 n}}\right]
\end{aligned}
$$




$$
\begin{aligned}
m_{Y}(t ; n) & =e^{-t[\sqrt{2 / n}(n / 2)]}\left(1-\frac{2 t}{\sqrt{2 n}}\right)^{-n / 2}, t<\frac{\sqrt{2 n}}{2} \\
& =\left[e^{t \sqrt{2 / n}}-t \sqrt{\frac{2}{n}} e^{t \sqrt{2 / n}]^{-n / 2}}\right]
\end{aligned}
$$

De acordo com a förmula de Taylor, existe um nümero $\xi(n)$, entre 0 e $t \sqrt{2 / n}$ tal que:

$$
e^{t \sqrt{2 / n}}=1+t \sqrt{\frac{2}{n}}+\frac{1}{2}\left(t \sqrt{\frac{2}{n}}\right)^{2}+\frac{e^{\xi(n)}}{6}\left(t \sqrt{\frac{2}{n}}\right)^{3}
$$

Se esta soma é substituída por $e^{t \sqrt{2 / n}}$ em $m_{Y}(t ; n)$, tem-se que

$$
m_{Y}(t, n)=\left(1-\frac{t^{2}}{n}+\frac{\psi(n)}{n}\right)^{-n / 2}
$$

com

$$
\psi(n)=\frac{\sqrt{2} t^{3} e^{\xi(n)}}{3 \sqrt{n}}-\frac{\sqrt{2} t^{3}}{\sqrt{n}}+\frac{2 t^{4}}{e^{\xi(n)}}
$$

Como $\xi(n)$ tende a zero, com $\underline{n}$ tendendo a infinito, en tão,

$$
\lim _{n \rightarrow \infty} \psi(n)=0,
$$

para todo valor fixo de $t$, e, de acordo com o limite fundamental (1) do Apêndice, tem-se que

$$
\lim _{n \rightarrow \infty} m_{Y}(t, n)=e^{t^{2} / 2},
$$

para todo $t$ real, que é a f.g.m. de uma distribuição normal padronizada. 
Conclui-se, pois, que a V.A. $Y_{n}=\frac{Z_{n}-n}{\sqrt{2 n}}$ tem uma dis tribuição normal padronizada como limite.

\subsubsection{4 - Aplicação da função geradora de momentos limite ao teorema do limite central}

Como se demonstra, pela utilização da técnica da fun ção geradora de momentos em (3.6.2.1.5), a média amostral $\bar{x}$, proveniente de uma população normal, de média m e variância $\sigma^{2}$, tem também distribuição normal, de média m e variância $\frac{\sigma^{2}}{n}$. Consequentemen te, a variävel aleatória $\frac{\bar{x}-m}{\sigma / \sqrt{n}}$ tem distribuição normal padrão.

Em teoria estatistica há um importante teorema chama do Teorema do Limite Central (vide Apêndice). Um caso especial desse teorema, afirma que, se $x_{1}, x_{2}, \ldots, x_{n}$ são os elementos de uma amostra aleatória de tamanho $\underline{n}$, provenientes de alguma distribuição que possui variância $\sigma^{2}$ e média $m\left(\sigma^{2}>0,-\infty<m<\infty\right)$, então a va riāvel aleatōria $\frac{\bar{x}-m}{\sigma / \sqrt{n}}$ tem uma distribuição normal limite com média zero e variância um. Pode-se, pois, usar essa distribuição normal aproximada para calcular probabilidades aproximadas concernentes a $\bar{x}$, o que se constitui na grande importância prática do conhecimento desse teorema.

Apresenta-se, nesta aplicação, somente uma forma especial do teorema do limite central, que consiste em requerer-se a existência de uma função geradora de momentos para a distribuição a partir da qual toma-se a amostra, o que, entretanto, é muito mais 
severo do que meramente requerer-se uma variância positiva para a distribuição. Segue-se a forma modificada do teorema do limite central.

Teorema: Sejam $x_{1}, x_{2}, \ldots, x_{n}$ os elementos de uma amostra alea tória provenientes de uma população com média m e variância $\sigma^{2}$, a qual possui função geradora de momentos $m_{X}(t)$ que existe para $-\mathrm{h}<\mathrm{t}<\mathrm{h}, \mathrm{h}>0$. Então a variävel aleatöria $\mathrm{Y}_{\mathrm{n}}=\frac{\overline{\mathrm{X}}-\mathrm{m}}{\sigma / \sqrt{\mathrm{n}}}$ tem uma distribuição limite, a qual é normal, com média zero e variância um.

\section{Demonstração:}

$$
\text { Como } m_{X}(t)=E\left[e^{t X}\right] \text { existe para }-h<t<h, h>0,
$$

a função $M_{X}(t)=E\left[e^{t(X-m)}\right]=e^{-m t} m_{X}(t)$ existe para $-h<t<h$, $h>0$.

Mostra-se inicialmente que:

$$
M_{X}(t)=1+\frac{\sigma^{2} t^{2}}{2}+\frac{\left[M^{\prime \prime}(\xi)-\sigma^{2}\right] t^{2}}{2}
$$

onde $\xi$ está entre zero e um. Para tanto, nota-se que

$$
\begin{aligned}
& M_{X}^{\prime}(t)=e^{-m t} m_{X}^{\prime}(t)-m e^{-m t} m_{X}(t) \\
& M_{X}^{\prime \prime}(t)=e^{-m t} m_{X}^{\prime \prime}(t)-2 m e^{-m t} m_{X}^{\prime}(t)+m^{2} e^{-m t} m_{X}(t)
\end{aligned}
$$

e que

$$
\mathrm{m}_{\mathrm{X}}(0)=1 \quad ; \quad \mathrm{m}_{\mathrm{X}}^{\prime}(0)=\mathrm{m} \quad \text { e } \quad \mathrm{m}_{\mathrm{X}}^{\prime \prime}(0)=\sigma^{2}+\mathrm{m}^{2}
$$


de forma que

$$
M_{X}(0)=1, \quad M_{X}^{\prime}(0)=(0) \text { e } \operatorname{Mg}_{X}^{\prime \prime \prime}(0)=\sigma^{2}
$$

Como pela fórmula de Taylor existe um nümero $\xi$ entre 0 e $t$ tal que

$$
\begin{aligned}
M_{X}(t) & =M_{X}(0)+M_{X}^{\prime}(0) t+\frac{M_{X}^{\prime \prime}(\xi) t^{2}}{2} \\
& =1+\frac{M_{X}^{\prime \prime}(\xi) t}{2}
\end{aligned}
$$

adicionando-se e subtraindo-se $\frac{\sigma^{2} t^{2}}{2}$ obtém-se então o que se preten de mostrar inicialmente, ou seja, que

$$
M_{X}(t)=1+\frac{\sigma^{2} t^{2}}{2}+\frac{\left[M_{X}^{\prime \prime}(\xi)-\sigma^{2}\right] t^{2}}{2}
$$

A seguir, considera-se $m_{Y}(t ; n)$, onde:

$$
\begin{aligned}
m_{Y}(t ; n) & =E\left[e^{t Y_{n}}\right]=E\left[e^{[t(\bar{X}-m) /(\sigma / \sqrt{n})]}\right] \\
\text { substituindo-se } Y_{n}= & \frac{\bar{x}-m}{\sigma / \sqrt{n}} \text { por } \frac{\sum X_{i}-n m}{\sigma \sqrt{n}}
\end{aligned}
$$

obtēm-se:

$$
m_{Y}(t ; n)=E\left[e^{\left[t\left(\sum X-n m\right) /(\sigma \sqrt{n})\right]}\right]
$$




$$
\begin{aligned}
m_{Y}(t ; n) & =E\left\{e^{t\left[\left(x_{1}-m\right) /(\sigma \sqrt{n})\right]} e^{t\left[\left(x_{L}-m\right) /(\sigma \sqrt{n})\right]} \ldots e^{t\left[\left(x_{n}-m\right) /(\sigma \sqrt{n})\right]}\right\} \\
& =E\left\{e^{\left[t\left(x_{1}-m\right) /(\sigma \sqrt{n})\right]}\right\} \ldots E\left\{e^{\left[t\left(x_{n}-m\right) /(\sigma \sqrt{n})\right]}\right\} \\
& =\left\{E\left[e^{t[(x-m) /(\sigma \sqrt{n})]}\right]\right\}
\end{aligned}
$$

$$
m_{Y}(t ; n)=\left[M_{X}\left(\frac{t}{\sigma \sqrt{n}}\right)\right]^{n} \quad, \quad-h<\frac{t}{\sigma \sqrt{n}}<h
$$

$$
\text { No resultado (8), substituindo-se } t \text { por } \frac{t}{\sigma \sqrt{n}} \text {, obtëm- }
$$

-se:

$$
M_{X}\left(\frac{t}{\sigma \sqrt{n}}\right)=1+\frac{t^{2}}{2 n}+\frac{\left[M_{X}^{\prime \prime}(\xi)-\sigma^{2}\right] t^{2}}{2 n \sigma^{2}}
$$

onde $\xi$ está entre 0 e $\frac{t}{\sigma \sqrt{n}}$, com $-h \sigma \sqrt{n}<t<h \sigma \sqrt{n}$.

$$
\text { E, a seguir, substituindo-se o resultado (10) em (9), }
$$

tem-se

$$
m_{Y}(t ; n)=\left\{1+\frac{t^{2}}{2 n}+\frac{\left.\left[M_{X}^{\prime \prime}(\xi)-\sigma^{2}\right] t^{2}\right\}}{2 n \sigma^{2}}\right\}
$$

Sendo $M_{X}^{\prime \prime}(t)$ continua para $t=0$, e desde que $\xi \rightarrow 0$, quando $\mathrm{n} \rightarrow \infty$, pelo limite fundamental (1) do Apêndice, tem-se que

$$
\lim _{n \rightarrow \infty}\left[M_{X}^{\prime \prime}(\xi)-\sigma^{2}\right]=0
$$

de forma que 


$$
\lim _{n \rightarrow \infty} m_{Y}(t ; n)=\lim _{n \rightarrow \infty}\left(1+\frac{t^{2}}{2 n}\right)^{n}=e^{t^{2} / 2}
$$

para todo $t$ real, a qual é a função geradora de momentos de uma dis tribuição normal padronizada.

Prova-se, pois, por esse resultado, que a variāvel aleatória $Y_{n}=\frac{\bar{X}-m}{\sigma / \sqrt{n}}$ tem distribuição normal padronizada como dis tribuição 1 imite.

\section{7 - Funções Caracterîsticas}

\subsection{1 - Definição}

Define-se função característica de uma variāvel alea tória $X$, e nota-se por $\phi_{X}(t)$ a

$$
\begin{aligned}
& \phi_{X}(t)=E\left[e^{i t x}\right]=\sum_{X} e^{i t x} f_{X}(x)=\sum_{X}\left[\cos (t x) f_{X}(x)+i \operatorname{sen}(t x) f_{X}(x)\right] \\
& =\sum_{X}\left[\cos (t x) f_{X}(x)\right]+i \sum_{X}\left[\operatorname{sen}(t x) f_{X}(x)\right],
\end{aligned}
$$

se $x$ é V.A. discreta, e

$$
\begin{aligned}
\phi_{X}(t)=E\left[e^{i t x}\right] & =\int_{-\infty}^{+\infty} e^{i t x} f_{X}(x) d x \\
& =\int_{-\infty}^{+\infty}\left[\cos (t x) f_{X}(x)+i \operatorname{sen}(t x) f_{X}(x)\right] d x \\
& =\int_{-\infty}^{+\infty} \cos (t x) f_{X}(x) d x+i \int_{-\infty}^{+\infty} \operatorname{sen}(t x) f_{X}(x) d x
\end{aligned}
$$


se $x$ é V.A. contínua, onde:

$$
\begin{aligned}
& f_{X}(x) \quad \bar{e} \text { a densidade de probabilidade de } x, \\
& e^{i t x}=\cos (t x)+i \operatorname{sen}(t x) \\
& i=\sqrt{-1} \quad, \quad \text { (operador complexo) }
\end{aligned}
$$

A funçáo característica $\phi_{X}(t)$ pode tambëm ser chamada "transformada de Fourier" de $\mathrm{f}_{X}(\mathrm{x})$.

Observa-se que a função característica sempre existe, pois a integral (ou somatório) que a define sempre converge como demonstra-se facilmente para variáveis contínuas (o mesmo pode ser verificado para o caso discreto, trabalhando-se com somatórios).

\section{Demonstração:}

Sendo $\mathrm{f}_{X}(\mathrm{x})$ densidade de probabilidade, $\mathrm{f}_{X}(\mathrm{x}) \geqslant 0$, para todo x real,

$$
\left|\phi_{X}(t)\right|=\left|\int_{-\infty}^{+\infty} e^{i t x} f_{X}(x) d x\right| \leq \int_{-\infty}^{+\infty}\left|e^{i t x}\right|\left|f_{X}(x)\right| d x
$$

e como $\left|e^{i t x}\right|=\cos ^{2}(t x)+\operatorname{sen}^{2}(t x)=1$, obtém-se que

$$
\left|\phi_{X}(t)\right|=\left|\int_{-\infty}^{+\infty} e^{i t x} f_{X}(x) d x\right| \leq \int_{-\infty}^{+\infty} f_{X}(x) d x=1
$$

o que demonstra a convergência absoluta dessa integral. 
3.7.2 - Obtenção de momentos a partir da função caracterîs tica

Os momentos de uma distribuição, podem tambëm ser ob tidos a partir da correspondente função caracterïstica, como mostra-se nesse estudo, seja por desenvolvimento da série $e^{i t x}$ ou por derivação sucessiva.

\subsubsection{1 - Obtenção dos momentos por desenvolvimen- to em sêrie da funçäo característica}

Partindo-se da definição dada para $\phi_{X}(t)$, no caso con tínuo (salientando-se que o mesmo pode ser visto para o caso discre to),

$$
\phi_{X}(t)=E\left[e^{i t x}\right]=\int_{-\infty}^{+\infty} e^{i t x} f_{X}(x) d x
$$

e desenvolvendo-se, sob o sinal de integração, a sërie $e^{i t x}$, obtëm-se:

$$
\begin{aligned}
\phi_{X}(t) & =\int_{-\infty}^{+\infty}\left[1+\frac{i t x}{1 !}+\frac{(i t x)^{2}}{2 !}+\frac{(i t x)^{3}}{3 !}+\ldots\right] f_{X}(x) d x \\
& =\int_{-\infty}^{+\infty} f_{X}(x) d x+\frac{i t}{1} \int_{-\infty}^{+\infty} x f_{X}(x) d x+\frac{(i t)^{2}}{2 !} \int_{-\infty}^{+\infty} x^{2} f_{X}(x) d x+\ldots
\end{aligned}
$$

e como, por definição de momentos, 


$$
\begin{aligned}
& \mu_{0}^{\prime}=1 \\
& \mu_{1}^{\prime}=\int_{-\infty}^{+\infty} x f_{X}(x) d x \\
& \mu_{2}^{\prime}=\int_{-\infty}^{+\infty} x^{2} f_{X}(x) d x \\
& \ldots . \\
& \mu_{r}^{\prime}=\int_{-\infty}^{+\infty} x^{r} f_{X}(x) d x
\end{aligned}
$$

nota-se que

$$
\phi_{X}(t)=\mu_{0}^{\prime}+\frac{i t}{1} \mu_{1}^{\prime}+\frac{(i t)^{2}}{2 !} \mu_{2}^{\prime}+\ldots+\frac{(i t)^{r}}{r !} \mu_{T}^{\prime}+\ldots
$$

Com isso, mostra-se que os momentos de uma distribui çáo podem ser obtidos a partir do desenvolvimento em série da funçäo caracteristica, pois

$$
\phi_{X}(t)=\sum_{j=0}^{\infty} \frac{1}{j !} \mu_{j}^{\prime}(i t)^{j}
$$

e que a função característica $\phi_{X}(t)$ é tambēm uma função geradora de momentos. 
131.

3.7.2.2 - Obtenção dos momentos por derivação sucessiva

$\mathrm{Se}$

$$
\phi_{X}(t)=\int_{-\infty}^{+\infty} e^{i t x} f_{X}(x) d x,
$$

a sua derivada primeira em relação a t é dada por:

$$
\frac{d}{d t}\left[\phi_{X}(t)\right]=i \int_{-\infty}^{+\infty} e^{i t x} x f_{X}(x) d x
$$

Multiplicando-se membro a membro essa igualdade por

-i, obtēm-se

$$
-i \frac{d}{d t}\left[\phi_{X}(t)\right]=\int_{-\infty}^{+\infty} e^{i t x} x f_{X}(x) d x,
$$

de modo que

$$
-\left.i \frac{d}{d t}\left[\phi_{X}(t)\right]\right|_{t=0}=\int_{-\infty}^{+\infty} x f_{X}(x) d x=E(X)=\mu_{1}^{\prime}
$$

A derivada segunda de $\phi_{X}(t)$ em relação a t é dada por

$$
\frac{d^{2}}{d t^{2}}\left[\phi_{X}(t)\right]=i^{2} \int_{-\infty}^{+\infty} e^{i t x} x^{2} f_{X}(x) d x
$$

Multiplicando-se membro a membro essa igualdade por

$-i^{2}$, obtēm-se:

$$
-\left.i^{2} \frac{d^{2}}{d t^{2}}\left[\phi_{X}(t)\right]\right|_{t=0}=\int_{-\infty}^{+\infty} x^{2} f_{X}(x) d x=E\left(X^{2}\right)=\mu_{2}^{\prime}
$$


Generalizando-se essa sequência de resultados, tem-se a expressão geral para o momento de ordem $r$, em relação à origem, a partir da função característica da distribuição, que é

$$
\mu_{r}^{\prime}=E\left[X^{r}\right]=\left.(-1)^{r} i^{r} \frac{d^{r}}{d t^{r}}\left[\phi_{X}(t)\right]\right|_{t=0}
$$

E necessärio observar-se que, embora o desenvolvimen to tenha sido feito para distribuições continuas, ele se aplica igualmente à distribuições discretas.

\subsubsection{3 - CālcuTo de momentos atravēs da função ca racteristica: distribuição gama}

Ilustrando o cálculo de momentos a partir da função característica, seja por desenvolvimento da série $e^{i t x}$, ou por deri vação sucessiva de $\phi_{X}(t)$, apresenta-se a distribuição gama.

Inicialmente, calcula-se a função caracteristica cor respondente a uma V.A. com distribuição gama com função de densidade de probabilidade

$$
\left\{\begin{array}{c}
f_{X}(x)=\frac{\lambda}{\Gamma(r)}(\lambda x)^{r-1} e^{-\lambda x}=\frac{\lambda^{r}}{\Gamma(r)} x^{r-1} e^{-\lambda x}, I_{[0, \infty)}(x) \\
r>0 ; \quad \lambda>0
\end{array}\right.
$$

como a seguir? 


$$
\begin{aligned}
\phi_{X}(t) & =E\left[e^{i t x}\right]=\frac{\lambda^{r}}{\Gamma(r)} \int_{0}^{\infty} e^{i t x} x^{r-1} e^{-\lambda x} d x \\
& =\frac{\lambda^{r}}{\Gamma(r)} \int_{0}^{\infty} x^{r-1} e^{-x(\lambda-i t)} d x
\end{aligned}
$$

Substituindo-se na igualdade acima $x(\lambda-i t)=w$, tem-

-se que

$$
\begin{aligned}
\phi_{X}(t) & =\frac{\lambda^{r}}{\Gamma(r)} \int_{0}^{\infty} \frac{w^{r-1}}{(\lambda-i t)^{r-1}} e^{-w} \frac{d w}{(\lambda-i t)} \\
& =\frac{\lambda^{r}}{\Gamma(r)(\lambda-i t)^{r}} \int_{0}^{\infty} e^{-w} w^{r-1} d w
\end{aligned}
$$

e, como

$$
\Gamma(r)=\int_{0}^{\infty} e^{-w} w^{r-1} d w
$$

obtēm-se, finalmente, a função característica

$$
\phi_{X}(t)=\left(\frac{\lambda}{\lambda-i t}\right)^{r}=\left(1-\frac{i t}{\lambda}\right)^{-r} .
$$

A seguir, desenvolve-se essa função obtida, $\phi_{X}(t)$, pe 10 binômio de Newton.

$$
\begin{aligned}
\phi_{X}(t)=\left(1-\frac{i t}{\lambda}\right)^{-r}=1 & +\frac{r}{\lambda} i t+\frac{r(r+1)}{\lambda^{2}} \frac{(i t)^{2}}{2 !}+ \\
& +\frac{r(r+1)(r+2)}{\lambda^{3}} \frac{(i t)^{3}}{3 !}+\ldots
\end{aligned}
$$

e 1 embrando que 


$$
\phi_{X}(t)=\sum_{j=0}^{\infty} \frac{1}{j^{\prime}} \mu_{j}^{\prime}(i t)^{j}
$$

tem-se os momentos desejados, ou seja,

$$
\begin{aligned}
& \mu_{0}^{\prime}=1 \\
& \mu_{1}^{\prime}=\frac{r}{\lambda} \\
& \mu_{2}^{\prime}=\frac{r(r+1)}{\lambda^{2}} \\
& \mu_{3}^{\prime}=\frac{r(r+1)(r+2)}{\lambda^{3}} \\
& \ldots= \\
& \mu_{n}^{\prime}=\frac{r(r+1)(r+2) \ldots(r+n-1)}{\lambda^{n}} \\
& \ldots .
\end{aligned}
$$

Lógicamente, a partir desses resultados, podem ser obtidos os momentos em relação à média, através da utilização da re 1 ação geral entre os momentos.

Alternativamente, os momentos de uma distribuição ga ma podem ser obtidos por derivação sucessiva, pois

$$
\mu_{n}^{r}=E\left(X^{n}\right)=\left.(-1)^{n}{ }_{i}^{n} \frac{d^{n}}{d t^{n}}\left[\phi_{X}(t)\right]\right|_{t=0}
$$

tal que 


$$
\begin{aligned}
& \mu_{1}^{\prime}=\left.(-1) i \frac{d}{d t}\left[\left(1-\frac{i t}{\lambda}\right)^{-r}\right]\right|_{t=0}=\frac{r}{\lambda} \\
& \mu_{2}^{\prime}=\left.(-1)^{2} i^{2} \frac{d^{2}}{d t^{2}}\left[\left(1-\frac{i t}{\lambda}\right)^{-r}\right]\right|_{t=0}=\frac{r(r+1)}{\lambda^{2}}
\end{aligned}
$$

e assim por diante

3.7.3 - Algumas propriedades das funções características

$$
3.7 .3 .1-\phi_{X}(0)=1
$$

pois,

$$
\begin{aligned}
\phi_{X}(0)=E\left[e^{0}\right]=E(1)=1 \\
\\
3.7 .3 .2-\left|\phi_{X}(t)\right|=1
\end{aligned}
$$

pois,

$$
\begin{aligned}
\left|\Phi_{X}(t)\right|= & \left|E\left(e^{i t x}\right)\right| \leq E\left|e^{i t x}\right|=E(1)=1 \\
& 3.7 .3 .3-\phi_{X}(t) \quad \bar{e} \text { uniformemente continua }
\end{aligned}
$$

pois,

$$
\begin{aligned}
\left|\phi_{X}(t+h)-\phi_{X}(t)\right| & =\left|E\left[e^{i(t+h) x}-e^{i t x}\right]\right| \\
& =\left|E\left[e^{i t x}\left(e^{i h x}-1\right)\right]\right| \leq E\left|e^{i t x}\left(e^{i h x}-1\right)\right| \\
& =E\left|e^{i h x}-1\right|
\end{aligned}
$$


136.

$\varepsilon$

$$
\begin{gathered}
\lim _{h \rightarrow 0}\left|\phi_{X}(t+h)-\phi_{X}(t)\right| \leq \lim _{h \rightarrow 0} E\left|e^{i h x}-1\right|=E\left[\lim _{h \rightarrow 0}\left|e^{i h x}-1\right|\right]=0 \\
3.7 .3 .4-\phi_{X+d}(t)=e^{i t d} \phi_{X}(t) \text {, d constante }
\end{gathered}
$$

pois,

$$
\begin{aligned}
\phi_{X+d}(t) & =E\left[e^{i t(x+d)}\right]=E\left[e^{i t x} e^{i d t]}=e^{i t d} E\left[e^{i t x}\right]\right. \\
& =e^{i t d} \Phi_{X}(t)
\end{aligned}
$$

$3.7 .3 .5-\phi_{c X}(t)=\phi_{X}(c t), c$ constante

pois,

$$
\begin{gathered}
\phi_{c X}(t)=E\left[e^{i t(c x)}\right]=E\left[e^{i(c t) x}\right]=\phi_{X}(c t) \\
3.7 .3 .6-\phi_{c X+d}(t)=e^{i t d} \phi_{X}(c t)
\end{gathered}
$$

pois,

$$
\phi_{c x+d}(t)=E\left[e^{(c x+d) i t}\right]=E\left[e^{(c x) i t}\right] E\left[e^{d i t}\right]
$$


3.7.4 - Alguns teoremas básicos das funções caracterî́sticas 3.7.4.1 - Teorema da inversão (fórmula de inversão)

\subsubsection{1 - Caso continuo}

Uma razão importante para o estudo da função caracte-

ristica

$$
\phi_{X}(t)=E\left[e^{i t x}\right]=\int_{-\infty}^{+\infty} e^{i t x} f_{X}(x) d x
$$

è que ela representa a transformada de Fourier da função de densida de $f_{X}(x)$. Pela teoria das transformadas de Fourier pode-se determinar a funçáo de densidade a partir de sua função característica. De fato,

$$
f_{X}(x)=\lim _{h \rightarrow 0} \lim _{T \rightarrow \infty} \frac{1}{2 \pi} \int_{-T}^{T} \frac{1-e^{-i t h}}{i t h} e^{-i t x} \phi_{X}(t) d t
$$

e, em particular, se

$$
\int_{-\infty}^{+\infty}\left|\phi_{X}(t)\right| d t<\infty \quad
$$

então,

$$
f_{X}(x)=\frac{1}{2 \pi} \int_{-\infty}^{+\infty} e^{-i t x} \phi_{X}(t) d t,
$$

se $f_{X}(x)$ é contỉnua (ROUSSAS, 1973). 
Demonstração:

- Seja $\phi_{X}(t)$ a funçào característica da função de densidade $f_{X}(x)$, cuja função de distribuição ē $F(x)$. Então,

$$
F(a+h)-F(a)=\lim _{T \rightarrow \infty} \frac{1}{2 \pi} \int_{-T}^{T} \frac{1-e^{-i t h}}{i t} e^{-i t a} \phi_{X}(t) d t
$$

sob a condição de que a e $a+h(c o m h>0)$ sejam pontos de continuidade de $F(x)$.

$x=$ hy, obtëm-se

$$
\text { - Seja } \lambda(h, T)=\frac{1}{\pi} \int_{0}^{T} \frac{\text { sen hy }}{y} d y, e \text {, substituindo-se }
$$

$$
\lambda(h T)=\frac{1}{\pi} \int_{0}^{h T} \frac{\text { sen } x}{x} d x
$$

que, de acordo com o lema 1 (Vide Apêndice) é limitada para todo $h$ e todo $\mathrm{T}$ e tal que

$$
\begin{gathered}
\lambda(-h T)=-\lambda(h T) \\
\operatorname{Iim}_{T \rightarrow \infty} \lambda(h T)=\left\{\begin{array}{cc}
1 / 2 & \text { se } h>0 \\
0 & \text { se } h=0 \\
-1 / 2 & \text { se } h<0
\end{array}\right.
\end{gathered}
$$

e,

- Introduzindo-se a integral

$$
J_{T}=\frac{1}{2 \pi} \int_{-T}^{T} \frac{e^{-i t a}-e^{-i t(a+h)}}{i t} \phi_{X}(t) d t
$$


e nela substituindo-se $\Phi_{X}(t)$ por $\int_{-\infty}^{+\infty} e^{i t x} f_{X}(x) d x$, obtém-se

$$
J_{T}=\frac{1}{2 \pi} \int_{-\infty}^{+\infty}\left[\int_{-T}^{T} \frac{e^{i t(a-x)}-e^{-i t(a+h-x)}}{i t} d t\right] f_{X}(x) d x
$$

e substituindo os exponenciais pelas respectivas funções trigonomëtricas,

$$
J_{\mathrm{T}}=\frac{1}{\pi} \int_{-\infty}^{+\infty}\left[\int_{0}^{\mathrm{T}} \frac{\operatorname{sen} t(x-a)-\operatorname{sen} t(x-a-h)}{t} d t\right] f_{X}(x) d x
$$

de forma que se pode escrever que

$$
g(x, T)=\lambda(x-a ; T)-\lambda(x-a-h ; T)
$$

e, a partir da limitação de $\lambda(x, T)$ e de (12), observa-se que $g(x, T)$ é também Iimitada para todo $\underline{x}$ e todo $\underline{T}$, de forma que

$$
\lim _{T \rightarrow \infty} g(x, T)=\left\{\begin{array}{cl}
0 & \text { se } x<a \\
1 / 2 & \text { se } x=a \\
1 & \text { se } a<x<a+h \\
1 / 2 & \text { se } x=a+h \\
0 & \text { se } x>a+h
\end{array}\right.
$$

Além disso, (13) pode ser escrita como:

$$
J_{T}=\int_{-\infty}^{+\infty} g(x, T) f_{X}(x) d x
$$


- Seja $\varepsilon$ um número positivo arbitrário. Como se admite que a e ath são pontos de continuidade de $F(x)$, e se demonstra que $g(x, T)$ é limitada, è possîvel selecionar um $\delta>0$ tão pequeno que são válidas simultaneamente as três inequações

$$
\begin{aligned}
& \left(I_{i}\right) \quad\left|\int_{a-\delta}^{a+\delta} g(x, T) f_{X}(x) d x\right| \leq \varepsilon \\
& \left(I_{2}\right) \quad\left|\int_{a+h-\delta}^{a+h+\delta} g(x, T) f_{X}(x) d x\right| \leq \varepsilon \\
& \left(I_{3}\right) \quad\left|\int_{a+\delta}^{a+h-\delta} f_{X}(x) d x-\int_{a}^{a+h} f_{X}(x) d x\right| \leq \varepsilon
\end{aligned}
$$

Alëm disso, a partir de (14), pode-se escolher $\mathrm{T}$ tão grande que as inequações

$$
\begin{aligned}
& \left(I_{4}\right)\left|\int_{a+\delta}^{a+h-\delta} g(x, T) f_{X}(x) d x-\int_{a+\delta}^{a+h-\delta} f_{X}(x) d x\right| \leq \varepsilon \\
& \left(I_{5}\right) \quad\left|\int_{-\infty}^{a-\delta} g(x, T) f_{X}(x) d x\right|+\left|\int_{a+h+\delta}^{\infty} g(x, T) f_{X}(x) d x\right| \leq \varepsilon
\end{aligned}
$$

são ambas satisfeitas.

- Decompõe-se, dessa forma, o intervalo de integração da integral (15) dentro de 5 sub-intervalos $(-\infty, a-\delta) ;(a-\delta ; a+\delta)$; $(a+\delta ; a+h-\delta) ;(a+h-\delta ; a+h+\delta)$ e $(a+h+\delta ; \infty)$ e obtém-se, usando $\left(I_{1}\right) ;\left(I_{2}\right)$ e $\left(I_{5}\right)$ 
141.

$$
\left(I_{6}\right) \quad\left|J_{T}-\int_{a+\delta}^{a+h-\delta} g(x, T) f_{X}(x) d x\right| \leq 3 \varepsilon
$$

e, a partir de $\left(I_{6}\right),\left(I_{4}\right)$ e $\left(I_{3}\right)$, e de $T$ escolhido suficientemente grande, que

$$
\begin{aligned}
& \qquad\left|J_{T}-\int_{a}^{a+b} f_{X}(x) d x\right| \leq 5 \varepsilon \\
& E \text {, como (16) è equivalente a (11), demonstra-se o teo }
\end{aligned}
$$
rema da inversão.

- Admitindo-se que $F(x)$ é função de distribuição associada à densidade $f_{X}(x)$, tem-se que

$$
\begin{aligned}
& f_{X}(x)=\lim _{h \rightarrow 0} \frac{F(x+h)-F(x)}{h}=\frac{d F(x)}{d x}=F^{\prime}(x) \\
& E, \text { associando-se esse resultado com o resultado }
\end{aligned}
$$
tomando-se $a=x$, desde que $x$ seja um ponto qualquer de continuidade de $F(x)$, pode-se escrever finalmente que:

$$
\begin{aligned}
f_{X}(x) & =\lim _{h \rightarrow 0} \frac{F(x+h)-F(x)}{h} \\
& =\lim _{h \rightarrow 0} \lim _{T \rightarrow \infty} \frac{1}{2 \pi} \int_{-T}^{T} \frac{1-e^{-i t h}}{i t h} e^{-i t x} \phi_{X}(t) d t
\end{aligned}
$$




\subsubsection{2 - Caso discreto}

De forma análoga, pode-se mostrar, no caso discreto, que a função de probabilidades $f_{X}(x)$ pode ser obtida de

$$
\phi_{X}(t)=\sum_{X=0}^{n} e^{i t x} f_{X}(x)
$$

mediante o emprego das séries de Fourier (que constituem, para o ca so discreto, o análogo da integral de Fourier).

De fato, se $x$ è variävel aleatória discreta, tem-se

que

$$
f_{X}(x)=\lim _{T \rightarrow \infty} \frac{1}{2 T} \int_{-T}^{T} e^{-i t x} \phi_{X}(t) d t
$$

e, em particular, se x tem distribuição "1attice"(Vide Apêndice), então,

$$
f_{X}(x)=\frac{1}{2 \pi} \int_{-\pi}^{\pi} e^{-i t x} \phi_{X}(t) d t .
$$

\subsubsection{3 - Apticações do teorema da inversão}

A aplicação fundamental do teorema da inversão,é que ele permite que se obtenha a função de densidade de probabilidade de uma distribuição, a partir da correspondente função característi ca, seja a distribuição de variável contínua, como em (3.7.4.1.1), ou de variável discreta (3.7.4.1.2). Apresenta-se, pois, exemplos dessa inversão, para o caso discreto ilustrado pela distribuiçăo bi nomial, e para o caso contínuo, pela distribuição normal padronizada. 


\subsection{1 - Caso discreto (distribuição binomial)}

Seja uma V.A. x com distribuição binomial e função caracteristica

$$
\phi_{X}(t)=\left(q+p e^{i t}\right)^{N} \quad(\text { veja-se } 3 \cdot 7 \cdot 6 \cdot 1 \cdot 2)
$$

Aplica-se o teorema da inversão a essa função característica de V.A. discreta, e obtēm-se a correspondente densidade

$$
f_{X}(x)=\left(\begin{array}{l}
N \\
X
\end{array}\right) p^{x} q^{N-x}
$$

como se segue:

$$
\begin{aligned}
& f_{X}(x)=\lim _{T \rightarrow \infty} \frac{1}{2 T} \int_{-T}^{T} e^{-i t x} \phi_{X}(t) d t \\
& =\lim _{T \rightarrow \infty}\left[\frac{1}{2 T} \int_{-T}^{T}\left(p e^{i t}+q\right)^{N} e^{-i t x} d t\right] \\
& =\lim _{T \rightarrow \infty}\left(\frac{1}{2 T} \int_{-T}^{T}\left[\sum_{r=0}^{N}\left(\begin{array}{l}
N \\
r
\end{array}\right)\left(p e^{i t}\right)^{r} q^{N-r} e^{-i t x}\right] d t\right\} \\
& =\lim _{T \rightarrow \infty}\left\{\frac{1}{2 T} \sum_{r=0}^{N}\left(\begin{array}{l}
N \\
r
\end{array}\right) p^{r} q^{N-r} \int_{-T}^{T} e^{i(r-x) t} d t\right\}
\end{aligned}
$$




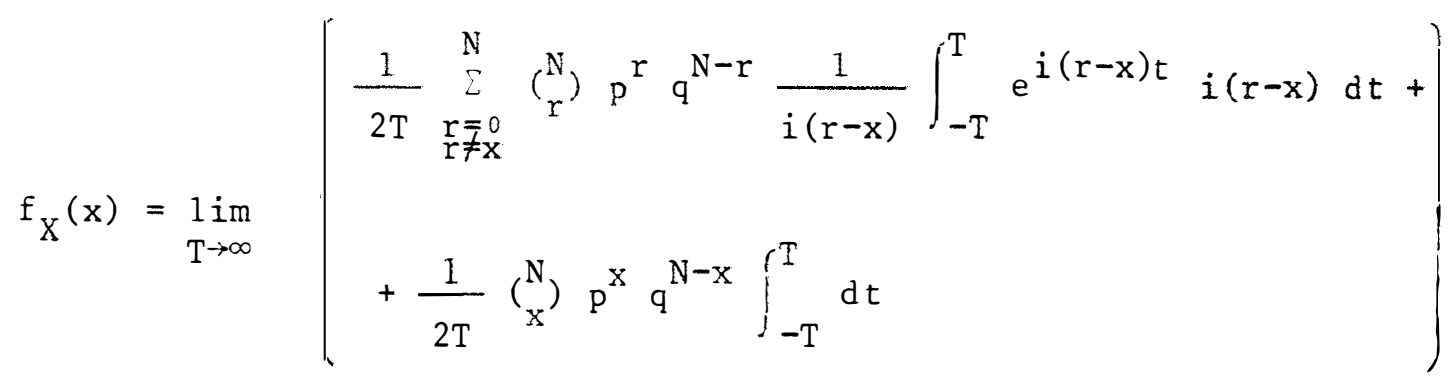

$$
\begin{aligned}
& =\lim _{\mathrm{T} \rightarrow \infty}\left\{\begin{array}{l}
\sum_{\substack{r \\
\mathrm{r} \neq \mathrm{x}}}^{N}\left(\begin{array}{l}
N \\
\mathrm{x}
\end{array}\right) \mathrm{p}^{r} q^{N-r} \frac{e^{i(r-x) T}-e^{-i(r-x) T}}{2 T j(r-x)}+ \\
+\frac{1}{2 T}\left(\begin{array}{l}
\mathrm{N} \\
\mathrm{x}
\end{array}\right) \mathrm{p}^{\mathrm{x}} \mathrm{q}^{\mathrm{N}-\mathrm{x}} 2 \mathrm{~T}
\end{array}\right\}
\end{aligned}
$$

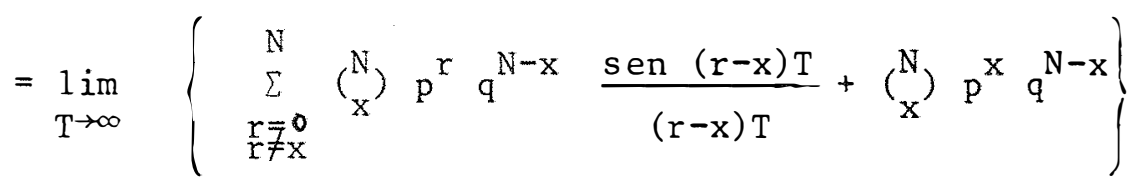

$$
\begin{aligned}
& =\left(\begin{array}{l}
N \\
X
\end{array}\right) p^{X} q^{N-x}
\end{aligned}
$$

que é a função densidade de uma distribuição binomial.

\subsection{2 - Caso continuo (distribuiçao normal padronizada)}

Seja uma V.A. x com distribuição normal padrão, $\mathrm{x} \cap \mathrm{N}(0,1)$ e função característica,

$$
\phi_{X}(t)=e^{-t^{2} / 2}
$$


Aplica-se o teorema da inversão a essa função característica de V.A. contỉnua e obtēm-se a correspondente densidade

$$
f_{X}(x)=\frac{1}{\sqrt{2 \pi}} e^{-x^{2} / 2}
$$

como se segue.

Sendo

$$
\left|\phi_{X}(t)\right|=\left|e^{-t^{2} / 2}\right|=e^{-t^{2} / 2}
$$

e

$$
\int_{-\infty}^{+\infty}\left|\phi_{X}(t)\right| d t<\infty
$$

de acordo com 3.1.1 tem-se que

$$
\begin{aligned}
f_{X}(x) & =\frac{1}{2 \pi} \int_{-\infty}^{+\infty} e^{-i t x} \phi_{X}(t) d t \\
& =\frac{1}{2 \pi} \int_{-\infty}^{+\infty} e^{-i t x} e^{-t^{2} / 2} d t \\
& =\frac{1}{2 \pi} \int_{-\infty}^{+\infty} e^{-i / 2\left(t^{2}+2 i t x\right)} d t \\
& =\frac{1}{2 \pi} \int_{-\infty}^{+\infty} e^{-1 / 2\left[t^{2}+2 t(i x)+(i x)^{2}\right]} e^{1 / 2(i x)^{2}} d t \\
& =\frac{e^{-x^{2} / 2}}{\sqrt{2 \pi}} \int_{-\infty}^{+\infty} \frac{1}{\sqrt{2 \pi}} e^{-1 / 2(t+i x)^{2}} d t \\
& =\frac{e^{-x^{2} / 2}}{\sqrt{2 \pi}} \frac{1}{\sqrt{2 \pi}} \int_{-\infty}^{+\infty} e^{-u^{2} / 2} d u
\end{aligned}
$$


146.

$$
\begin{aligned}
f_{X}(x) & =\frac{e^{(-1 / 2) x^{2}}}{\sqrt{2 \pi}} \cdot 1 \\
& =\frac{1}{\sqrt{2 \pi}} e^{-x^{2} / 2}
\end{aligned}
$$

que è a função densidade de probabilidade de uma distribuição normal padronizada.

\subsubsection{2 - Teorema da unicidade}

Existe uma única função densidade de probabilidade as sociada a uma função característica, e vice-versa (ROUSSAS, 1973), ou seja, duas funções de distribuição $F_{1}(X)$ e $F_{2}(X)$ são idênticas, se e somente se, suas funções características $\phi_{1}(t)$ e $\phi_{2}(t)$ são idênticas .

Não se apresenta a prova dessa teorema, pois o mesmo

é decorrência imediata do teorema da inversão visto em (3.7.4.1).

\subsection{5 - Funções caracterīsticas para funções de vārias va- riāveis aleatörias}

Apresenta-se, de forma condensada, a definição e a1gumas propriedades das funções características de funções de várias variāveis, como generalização do que se apresenta neste trabalho pa ra funções de uma variävel independente. 
147.

\subsubsection{1 - Definiçăo}

Define-se função característica da V.A. conjunta $x_{1}$, $x_{2}, \ldots, x_{K}$, ou do vetor aleatörio $X=\left(x_{1} x_{2} \ldots x_{K}\right)$ e nota-se por $\phi_{X}(\boldsymbol{T})$ ou $\phi_{X_{1}}, \ldots, X_{K}\left(t_{1}, \ldots, t_{K}\right)$ a

$$
\phi_{X}(T)=E\left[e^{i} T^{\prime} X\right]
$$

$\operatorname{com} r=\left(\begin{array}{llll}t_{1} & t_{2} & \ldots & t_{K}\end{array}\right)$, ou

$$
\begin{gathered}
\phi_{X_{1}}, \ldots, x_{K}\left(t_{1}, \ldots, t_{K}\right)=E\left[e^{\left.i \sum_{j} t_{j} x_{j}\right]}=E\left[e^{\left.i t_{1} x_{1}+i t_{2} x_{2}+\ldots+i t_{K} x_{K}\right]}\right.\right. \\
j=1,2, \ldots, K
\end{gathered}
$$

A função característica conjunta $\phi_{X_{1}}, \ldots, x_{K}\left(t_{1}, \ldots, t_{K}\right)$ satisfaz propriedades e teoremas análogos aos apresentados para $\phi_{X}(t)$ como os que se seguem.

\subsubsection{2 - Propriedades}

$(3.7 .5 .2 .1) \quad \Phi_{\mathrm{X}_{1}}, \ldots, \mathrm{X}_{\mathrm{K}}(0, \ldots, 0)=1$

$(3.7 .5 .2 .2) \quad\left|\phi_{X_{1}}, \ldots, x_{K}\left(t_{1}, \ldots, t_{K}\right)\right| \leq 1$

(3.7.5.2.3) $\phi_{X_{1}, \ldots, X_{K}}\left(t_{1}, \ldots, t_{K}\right) \quad \bar{e}$ uniformemente contínua

$(3.7 .5 .2 .4) \quad \phi_{j}\left(x_{j}+d_{j}\right)\left(t_{1}, \ldots, t_{k}\right)=e^{i \sum_{j} t_{j} d_{j}} \phi_{x_{1}}, \ldots, x_{k}\left(t_{1}, \ldots, t_{k}\right)$

$$
j=1,2, \ldots, k \quad ; d \text { constante }
$$




$$
\begin{aligned}
& (3.7 .5 .2 .5) \phi_{j} c_{j} x_{j}\left(t_{1}, \ldots, t_{K}\right)=\phi_{x_{1}}, \ldots, x_{K}\left(c_{1} t_{1}, \ldots, c_{K} t_{K}\right) \\
& j=1,2, \ldots, k ; \quad c \text { constante } \\
& (3.7 .5 .2 .6) \phi_{j}\left(c_{j} x_{j}+d_{j}\right)\left(t_{1}, \ldots, t_{k}\right)=e^{i \sum_{j} t_{j} d} \phi_{X_{1}}, \ldots, x_{K}\left(c_{1} t_{1}, \ldots, c_{K} t_{K}\right) \\
& \mathrm{j}=1,2, \ldots, \mathrm{k} \quad ; \quad c \text { e d constantes }
\end{aligned}
$$

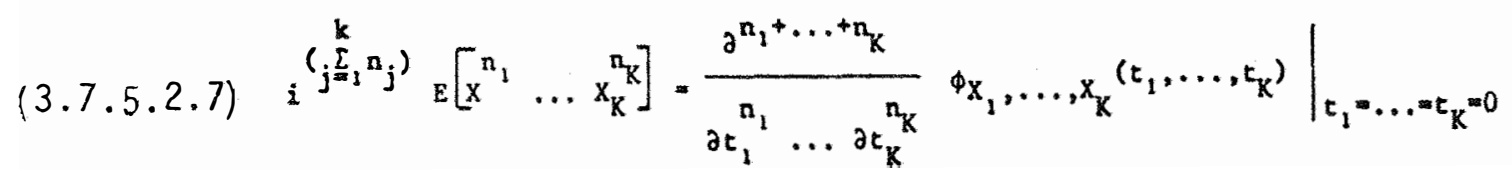

$$
\begin{aligned}
& (3.7 .5 .2 .8) i^{(n)} E\left(x_{j}^{n}\right)=\frac{\partial^{n}}{\partial t_{j}^{n}} \phi_{X_{1}}, \ldots,\left.x_{K}\left(t_{1}, \ldots, t_{K}\right)\right|_{t_{1}=\ldots, t_{k}=0} \\
& \text { 3.7.5.3 - Teoremas } \\
& \text { 3.7.5.3.1 - Teorema da inversão }
\end{aligned}
$$$$
\text { Se }\left(x_{1}, \ldots, x_{K}\right) \text { é uma V.A. conjunta discreta, }
$$

$$
\begin{aligned}
f_{X_{1}}, \ldots, x_{X}\left(x_{1}, \ldots, x_{K}\right)= & \lim _{T \rightarrow \infty}\left(\frac{1}{2 T}\right)^{k} \int_{-T}^{T} \ldots \int_{-T}^{T} e^{-i t_{1} x_{1}-\ldots-i t_{K} X_{K}} \times \\
& \times \phi_{X_{1}}, \ldots, x_{K}\left(t_{1}, \ldots, t_{K}\right) d t_{1} \ldots d t_{K}
\end{aligned}
$$


e, se $\left(X_{1}, \ldots, X_{K}\right) \vec{e}$ uma $V \cdot A$. conjunta continua,

$$
\begin{aligned}
& f_{X_{1}, \ldots, x_{K}}\left(x_{1}, \ldots, x_{K}\right)=\lim _{h \rightarrow 0} \lim _{T \rightarrow \infty}\left(\frac{1}{2 \pi}\right)^{k} \int_{-T}^{T} \cdots \int_{-T}^{T} \prod_{j=1}^{k}\left(\frac{1-e^{-i t_{j} h}}{i t_{j} h}\right) x \\
& e^{-i t_{1} x_{1}-\ldots-i t_{k} x_{K}} \quad x \\
& x \phi_{X_{1}}, \ldots, X_{K}\left(t_{1}, \ldots, t_{K}\right) d t_{I} \ldots d t_{K}
\end{aligned}
$$

$$
\int_{-\infty}^{+\infty} \ldots \int_{-\infty}^{+\infty}\left|\phi_{X_{1}}, \ldots, x_{K}\left(t_{1}, \ldots, t_{K}\right)\right| d t_{1} \ldots d t_{K}<\infty
$$

então,

$$
\begin{aligned}
& f_{x_{1}}, \ldots, x_{K}\left(x_{1}, \ldots, x_{K}\right)=\left(\frac{1}{2 \pi}\right)^{k} \int_{-\infty}^{+\infty} \ldots \int_{-\infty}^{+\infty} e^{-i t_{1} x_{1}-\ldots-i t_{K} x_{K}} \times \\
& \times \phi_{X_{1}}, \ldots, x_{K}\left(t_{1}, \ldots, t_{K}\right) d t_{1} \ldots d t_{K} \\
& \text { (ROUSSAS, 1973). }
\end{aligned}
$$

\subsubsection{2 - Teorema da unicidade}

Há uma correspondēncia biunívoca entre a função caracteristica e a funçäo densidade de probabilidade de uma V.A. conjunta $\left(X_{1}, \ldots, X_{K}\right)$ (ROUSSAS, 1973). 


\subsection{6 - Cấculo das funções caracterīsticas de algumas dis} tribuiçöes especiais de probabilidade

Também dentro do propósito inicial deste trabalho, calculam-se as funções características das distribuições mais usuais de probabilidade, cujas funções de densidade e correspondentes funções geradoras de momentos se apresentam em (3.4). Não se obtêm os momentos, como em (3.4) a partir das funções características, e lem bra-se apenas que o momento de ordem $\underline{r}$, em relaçăo à origem, pode ser obtido a partir da função característica, através de

$$
\mu_{r}^{\prime}=E\left[X^{r}\right]=\left.(-1)^{r} i^{r} \frac{d^{r}}{d t^{r}}\left[\phi_{X}(t)\right]\right|_{t=0}
$$

e os momentos em relação à média, pela relação geral entre os momen tos, dada por

$$
\mu_{r}=\sum_{j=0}^{r}\left(\begin{array}{l}
r \\
j
\end{array}\right) \mu_{r-j}^{\prime}\left(-\mu_{l}^{\prime}\right)^{j}
$$

\subsubsection{1 - Distribuiçōes discretas}

\subsubsection{1 - Distribuiçăo de Bernoulli}

Se uma V.A. discreta $\underline{x}$ tem distribuição de Bernoulli, com densidade 


$$
\begin{aligned}
& f_{X}(x)=f_{X}(x, p)= \begin{cases}p^{x}(1-p)^{1-x} & , \text { para } x=0 \text { ou } x=1 \\
0 & , \text { para outros valores }\end{cases} \\
& \operatorname{com} 0 \leq p \leq 1 \quad \text { e } q=1-p
\end{aligned}
$$

a sua função característica $\phi_{X}(t) \vec{e}$ dada por:

$$
\phi_{X}(t)=\left(q+p e^{i t}\right)
$$

pois,

$$
\begin{aligned}
\phi_{X}(t) & =E\left[e^{i t x}\right]=\Sigma\left[e^{i t x} p^{x} q^{1-x}\right] \\
& =\sum_{x=0}^{1} e^{i t x} p^{x} q^{1-x}=\sum_{x=0}^{1}\left(p e^{i t}\right)^{x} q^{1-x} \\
& =\left(q+p e^{i t}\right)
\end{aligned}
$$

\subsubsection{2 - Distribuição binomial}

Se uma V.A. discreta $x$ tem distribuição binomial, com

densidade

$$
\begin{gathered}
f_{X}(x)=f_{X}(x ; n, p)= \begin{cases}\left(\begin{array}{l}
N \\
x
\end{array}\right) p^{x} q^{N-x} \quad, \text { para } x=0,1,2, \ldots, N \\
0 \quad, \text { para outros valores }\end{cases} \\
\text { com } 0 \leq p \leq 1, \quad q=1-p
\end{gathered}
$$

a sua função característica $\phi_{X}(t)$ è dada por

$$
\phi_{X}(t)=\left(q+p e^{i t}\right)^{N} \text {, }
$$


pois,

$$
\begin{aligned}
\Phi_{X}(t) & =E\left[e^{i t x}\right]=\sum\left[e_{-i t x}\left(\begin{array}{l}
N \\
x
\end{array}\right) p^{x} q^{N-x}\right] \\
& =\sum_{x=0}^{N} e^{i t x}\left(\begin{array}{l}
N \\
x
\end{array}\right) p^{x} q^{N-x} \\
& =\sum_{x=0}^{N}\left(\begin{array}{l}
N \\
x
\end{array}\right)\left(p e^{i t}\right)^{x} q^{N-x} \\
& =\left(q+p e^{i t}\right)^{N}
\end{aligned}
$$

\subsubsection{3 - Distribuição de Poisson}

Se uma V.A. discreta $x$ tem distribuição de Poisson,

com densidade

$$
\begin{aligned}
& f_{X}(x)=f_{X}(x ; \lambda)=\left\{\begin{array}{cl}
\frac{e^{-\lambda} \lambda^{x}}{x !} & , \text { para } x=0,1,2, \ldots \\
0 & , \text { para outros valores }
\end{array}\right. \\
& \operatorname{com} \lambda>0 \text {, }
\end{aligned}
$$

a sua função característica $\phi_{X}(t) \bar{e}$ dada por

$$
\phi_{X}(t)=e^{\lambda\left(e^{i t}-1\right)}
$$

pois,

$$
\phi_{X}(t)=E\left[e^{i t x}\right]=\Sigma\left[e^{i t x} \frac{e^{-\lambda} \lambda^{x}}{x !}\right]
$$




$$
\begin{aligned}
\phi_{X}(t) & =e^{-\lambda} \sum_{x=0}^{\infty} \frac{e^{i t x} \lambda^{x}}{x !} \\
& =e^{-\lambda} \sum_{x=0}^{\infty} \frac{\left(\lambda e^{i t}\right)^{x}}{x !} \\
& =e^{-\lambda} e^{\lambda e^{i t}} \\
& =e^{\lambda\left(e^{i t}-1\right)}
\end{aligned}
$$

3.7.6.1.4 - Distribuição geométrica (ou Pascal)

Se uma variāvel aleatória discreta $x$ apresenta distribuição geométrica, com densidade

$$
\begin{aligned}
f_{X}(x)=f_{X}(x ; p)= \begin{cases}p(1-p)^{x} & , \text { para } x=0,1,2, \ldots \\
0 \quad & \text { para outros valores }\end{cases} \\
\quad \text { com } 0<p \leq 1 \quad ; \quad q=1-p,
\end{aligned}
$$

a sua função característica $\phi_{X}(t)$ é dada por

$$
\phi_{X}(t)=p\left[\frac{1}{1-q e^{i t}}\right] ;\left|q e^{i t}\right|<1
$$

pois,

$$
\begin{aligned}
\phi_{X}(t) & =E\left[e^{i t x}\right]=\sum\left[e^{i t x} p(1-p)^{x}\right] \\
& =p \sum_{x=0}^{\infty}\left[\left(q e^{i t}\right)^{\tilde{x}}\right]
\end{aligned}
$$




$$
\begin{aligned}
\phi_{X}(t) & =p\left[1+q e^{i t}+q^{2} e^{2 i t}+q^{3} e^{3 i t}+\ldots\right] \\
& =p\left[\frac{1}{1-q e^{i t}}\right] \quad ; \quad\left|q e^{i t}\right|<1
\end{aligned}
$$

\subsubsection{5 - Distribuição binomial nega- tiva}

Se uma V.A. discreta $x$ tem distribuição binomial negativa, com densidade

$$
\begin{gathered}
f_{X}(x)=f_{X}(x ; r, p)=\left\{\begin{array}{c}
\left(\begin{array}{c}
r+x-1 \\
x
\end{array}\right) p^{r} q^{x}=\left(\begin{array}{c}
-r \\
x
\end{array}\right) p^{r}(-q) \\
0 \quad \text {, para } x=0,1, \ldots
\end{array}\right. \\
\text { com } r=1,2,3, \ldots ; 0<p \leq 1 ; q=1-p
\end{gathered}
$$

a sua função característica $\phi_{X}(t)$ é dada por

$$
\phi_{X}(t)=\left[\frac{p}{1-q e^{i t}}\right]^{r}, \quad\left|q e^{i t}\right|<1
$$

pois,

$$
\begin{aligned}
\phi_{X}(t) & =E\left[e^{i t x}\right]=\sum\left[e^{i t x}\left(\begin{array}{c}
-r \\
x
\end{array}\right) p^{r}(-q) x\right] \\
& =p^{r} \sum_{0}^{\infty}\left(\begin{array}{c}
-r \\
x
\end{array}\right)\left(-q e^{i t}\right)^{x} \\
& =p^{r}\left(1-q e^{i t}\right)^{-r} \\
& =\left[\frac{p}{1-q e^{i t}}\right]^{r}, \quad\left|q e^{i t}\right|<1
\end{aligned}
$$




\subsubsection{6 - Distribuição multinomial}

Se uma V.A. discreta conjunta $\left(X_{1}, \ldots, x_{K}\right)$ apresenta distribuição multinomial, com densidade conjunta

$$
\begin{aligned}
& \mathrm{f}_{X_{1}}, \ldots, \mathrm{X}_{\mathrm{K}}\left(\mathrm{x}_{1}, \mathrm{x}_{2}, \ldots, \mathrm{x}_{\mathrm{K}}\right)=\frac{\mathrm{n} !}{\mathrm{x}_{1} ! \mathrm{x}_{2} ! \ldots \mathrm{x}_{\mathrm{K}} !} \mathrm{p}_{1}^{\mathrm{x}_{1}} \mathrm{p}_{2}^{\mathrm{x}_{2}} \ldots \mathrm{p}_{\mathrm{K}}^{\mathrm{x}_{k}} \\
& \operatorname{com} \quad \sum \mathrm{p}_{\mathrm{j}}=1 \quad ; \quad \sum \mathrm{x}_{\mathrm{j}}=\mathrm{n} \quad ; \quad \mathrm{j}=1,2, \ldots, \mathrm{k}
\end{aligned}
$$

a sua função caracterỉstica $\phi_{X}, \ldots, X_{K}\left(t_{1}, \ldots, t_{K}\right)$ é dada por

$$
\phi_{X_{1}}, \ldots, x_{K}\left(t_{1}, \ldots, t_{K}\right)=\left(p_{1} e^{i t_{1}}+\ldots+p_{X} e^{i t_{k}}\right)^{n}
$$

pois,

$$
\begin{aligned}
& \phi_{X_{1}}, \ldots, x_{K}\left(t_{1}, \ldots, t_{K}\right)=E\left[e^{i \sum_{j} t_{j} x_{j}}\right] \\
& =\sum_{x_{1}, \ldots, x_{K}} e^{i t_{1} x_{1}+\ldots+i t_{K} x_{K}} \frac{n !}{x_{1} ! \ldots x_{K} !} x \\
& \times \mathrm{p}_{1}^{\mathrm{x}_{1}} \ldots \mathrm{p}_{\mathrm{K}}^{\mathrm{x}_{\mathrm{K}}} \\
& =\sum_{x_{1}, \ldots, x_{K}} \frac{n !}{x_{1} ! \ldots x_{K} !}\left(p_{1} e^{i t_{1}}\right)^{x_{1}} \ldots\left(p_{K} e^{i t_{k}}\right)^{x_{K}} \\
& =\left(p_{1} e^{i t}+\ldots+p_{K} e^{i t_{K}}\right)^{n}
\end{aligned}
$$


3.7.6.2 - Distribuiçoes continuas

\subsubsection{1 - Distribuição retangular (ou uniforme)}

Se uma V.A. contỉnua $X$ tem distribuição retangular, com densidade

$$
\begin{aligned}
& f_{X}(x)=f_{X}(x ; a, b)=\frac{1}{b-a}, I_{[a, b]}(x) \\
& \text { com }-\infty<a<b<\infty,
\end{aligned}
$$

a sua função característica $\phi_{X}(t)$ é dada por

$$
\phi_{X}(t)=\frac{e^{i t b}-e^{i t a}}{i t(b-a)}
$$

pois,

$$
\begin{aligned}
\dot{\Phi}_{X}(t) & =E\left[e^{i t x}\right]=\int_{a}^{b} e^{i t x} \frac{1}{b-a} d x \\
& =\frac{1}{b-a} \int_{a}^{b} e^{i t x} d x \\
& =\frac{e^{i t b}-e^{i t a}}{i t(b-a)}
\end{aligned}
$$




\subsubsection{2 - Distribuição exponencia) (ne gativa)}

Se uma V.A. contínua $X$ tem distribuição exponencial

(negativa), com densidade

$$
f_{X}(x)=f_{X}(x ; \lambda)=\lambda e^{-\lambda x} \quad, \quad I_{[0, \infty)}(x)
$$

$\operatorname{com} \lambda>0$, a sua função característica $\phi_{X}(t) \bar{e}$ dada por

$$
\phi_{X}(t)=\frac{\lambda}{\lambda-i t} \quad, \quad \text { it }<\lambda
$$

po is :

$$
\begin{aligned}
\phi_{X}(t) & =E\left[e^{i t x}\right]=\int_{0}^{\infty} i t x \lambda e^{-\lambda x} d x \\
& =\lambda \int_{0}^{\infty} e^{i t x} e^{-\lambda x} d x \\
& =\lambda \int_{0}^{\infty} e^{x(i t-\lambda)} d x
\end{aligned}
$$

onde, substituindo-se $u=-x(i t-\lambda)$, obtém-se

$$
\begin{aligned}
\phi_{X}(t) & =\frac{\lambda}{\lambda-i t} \int_{0}^{\infty} e^{-u} d u \\
& =\frac{\lambda}{\lambda-i t},
\end{aligned}
$$

pois

$$
\int_{0}^{\infty} e^{-u} d u=\Gamma(1)=1
$$




\subsubsection{3 - Distribuição gama}

Se uma V.A. contínua $X$ apresenta distribuiçao gama,

com densidade

$$
f_{X}(x)=f_{X}(x ; r, \lambda)=\frac{\lambda}{\Gamma(r)}(\lambda x)^{r-1} e^{-\lambda x} \quad I_{[0, \infty)}(x)
$$

com $r>0, \lambda>0$, a sua função característica $\phi_{X}(t)$ é dada por

$$
\phi_{X}(t)=\left(\frac{\lambda}{\lambda-i t}\right)^{r} \quad, \quad \text { it }<\lambda
$$

pois,

$$
\begin{aligned}
\phi_{X}(t) & =E\left[e^{i t x}\right]=\int_{0}^{\infty} e^{i t x} \frac{\lambda}{\Gamma(r)}(\lambda x)^{r-1} e^{-\lambda x} d x \\
& =\frac{\lambda^{r}}{\Gamma(r)} \int_{0}^{\infty} e^{-x(\lambda-i t)} x^{r-1} d x \\
& =\frac{\lambda^{r}}{(\lambda-i t)^{r}} \frac{1}{\Gamma(r)}(\lambda-i t) \int_{0}^{\infty} e^{-x(\lambda-i t)}[x(\lambda-i t)]^{r-1} d x
\end{aligned}
$$

onde, substituindo-se $u=x(\lambda-i t)$, obtēm-se

$$
\phi_{X}(t)=\frac{\lambda^{r}}{(\lambda-i t)^{r}} \frac{1}{\Gamma(r)} \frac{1}{r} r \int_{0}^{\infty} e^{-u} u^{r-1} d u
$$

e, como

$$
r \int_{0}^{\infty} e^{-u} u^{r-1} d u=\Gamma(r+1)=r \Gamma(r)
$$

tem-se que

$$
\phi_{X}(t)=\left(\frac{\lambda}{\lambda-i t}\right)^{r} \quad, \quad i t<\lambda
$$


159.

\subsubsection{4 - Distribuição beta}

Se uma V.A. contỉnua $X$ apresenta distribuição beta, com densidade

$$
f_{X}(x)=f_{X}(x ; p, q)=\frac{1}{B(p, q)} x^{p-1}(1-x)^{q-1}, I_{(0,1)}(x)
$$

$\operatorname{com} \beta(p, q)=\frac{\Gamma(p) \Gamma(q)}{\Gamma(p+q)} ; p>0$ e $q>0$, a sua função característ ca è dada por:

$$
\phi_{X}(t)=\frac{\Gamma(p+q)}{\Gamma(p)} \sum_{j=0}^{\infty} \frac{\Gamma(p+j)}{\Gamma(p+q+j) \Gamma(j+1)} \quad(i t)^{j} \quad \text { (LUKACS, 1970) }
$$

\subsubsection{5 - Distribuição normal}

Se uma V.A. contínua $X$ apresenta distribuição normal, com densidade

$$
f_{X}(x)=f_{X}\left(x ; m, \sigma^{2}\right)=\frac{1}{\sigma \sqrt{2 \pi}} e^{-(x-m)^{2} /\left(2 \sigma^{2}\right)}, \quad-\infty<x<\infty
$$

com $-\infty<m<\infty, \sigma>0$, a sua funçäo característica é dada por

$$
\Phi_{X}(t)=e^{i t m-\frac{\sigma^{2} t^{2}}{2}}
$$

e, em particular, se

$$
x \cap N(0,1) \quad, \quad \phi_{X}(t)=e^{-t^{2} / 2}
$$

e, se

$$
x \cap N\left(0, \sigma^{2}\right) \quad, \quad \phi_{X}(t)=e^{(-1 / 2) t^{2} \sigma^{2}}
$$


160.

pois,

$$
\begin{aligned}
\phi_{X}(t) & =E\left[e^{i t x}\right]=\int_{-\infty}^{+\infty} e^{i t x} \frac{1}{\sigma \sqrt{2 \pi}} e^{-(x-m)^{2} /\left(2 \sigma^{2}\right)} d x \\
& =\frac{1}{\sigma \sqrt{2 \pi}} \int_{-\infty}^{+\infty} e^{i t x} e^{-(x-m)^{2} /\left(2 \sigma^{2}\right)} d x
\end{aligned}
$$

$$
\begin{aligned}
& \text { onde, substituindo-se u }=\frac{x-m}{\sigma} \text {, obtém-se } \\
& \qquad \begin{aligned}
\phi_{X}(t) & =\frac{1}{\sqrt{2 \pi}} \int_{-\infty}^{+\infty} e^{(-1 / 2) u^{2}+i t u \sigma+i t m} d u \\
& =\frac{1}{\sqrt{2 \pi}} e^{i t m} \int_{-\infty}^{+\infty} e^{-1 / 2\left[(u-i t \sigma)^{2}+t^{2} \sigma^{2}\right]} d u \\
& =\frac{e^{i t m}-\frac{t^{2} \sigma^{2}}{2}}{\sqrt{2 \pi}} \int_{-\infty}^{+\infty} e^{-1 / 2(u-i t \sigma)^{2}} d u
\end{aligned}
\end{aligned}
$$

e ef etuando-se a substituição $z=u-i t \sigma$ obtém-se finalmente que

$$
\begin{aligned}
\phi_{X}(t) & =\frac{e^{i t m}-\frac{t^{2} \sigma^{2}}{2}}{\sqrt{2 \pi}} \int_{-\infty}^{+\infty} e^{-z^{2} / 2} d z \\
& =e^{i t m-\left[\left(t^{2} \sigma^{2}\right) / 2\right]},
\end{aligned}
$$

pois

$$
\frac{1}{\sqrt{2 \pi}} \int_{-\infty}^{+\infty} e^{-z^{2} / 2} d z=1
$$


3.7.6.2.6 - Distribuição de qui-quadrado

Se uma V.A. continua $v_{f}$ tem distribuição de qui-quadrado, com $\underline{f}$ graus de 1 iberdade, com densidade

$$
f_{v_{f}}\left(v_{f}\right)=\frac{1}{2^{f} / 2} \frac{1}{\Gamma(f / 2)} e^{-v_{f} / 2} v_{f}(f-2) / 2,0 \leq v_{f} \leq \infty
$$

a sua função característica é dada por

$$
\phi_{\mathrm{f}}(t)=(1-2 i t)^{-f / 2},
$$

pois,

$$
\begin{aligned}
\phi_{\mathrm{f}}(t) & =E\left[e^{i t v}\right]=E\left[e^{i t\left(u_{1}^{2}+u_{2}^{2}+\ldots+u_{f}^{2}\right)}\right] \\
& =E\left[e^{i t u_{1}^{2}}\right] E\left[e^{i t u_{2}^{2}}\right] \ldots E\left[e^{i t u_{f}^{2}}\right]
\end{aligned}
$$

e, como $u_{i} \cap N(0,1)$,

$$
\begin{aligned}
E\left[e^{i t u_{i}^{2}} i\right] & =\frac{1}{\sqrt{2 \pi}} 2 \int_{0}^{\infty} e^{\left(u_{i}^{2} / 2\right)(1-2 i t)} d u_{i} \\
& =(1-2 i t)^{-1 / 2}
\end{aligned}
$$

tem-se que

$$
\phi_{v_{f}}(t)=(1-2 i t)^{-f / 2}
$$




\subsection{7 - O caso da distribuição de Cauchy}

Uma razão importante para se estudar a função carac-

terística é que ela sempre existe, como se demonstra no último parā grafo de (3.7.1). A função geradora, porēm, pode não existir, se $E\left[e^{t x}\right\rceil$ não existe. Logo, há distribuições de probabilidade que não possuem funções geradoras de momentos, possuindo, no entanto, função característica, como é o caso da distribuição de Cauchy.

\subsubsection{7 - Distribuição de Cauchy}

Uma variāvel aleatória $X$ distribui-se segundo Cauchy, ou tem distribuição de Cauchy, se a sua função densidade de prô babilidade é dada por

$$
f_{X}(x)=\frac{a}{\pi\left(x^{2}+a^{2}\right)}, a>0,-\infty<x<\infty .
$$

Esta função de densidade é simétrica em relação a $x=0$, de forma que, mesmo não possuindo média, pode-se considerä-la como zero. A variância e os momentos de ordem mais alta não existem, como também não existe a função geradora de momentos. Entretanto, a função característica existe, e é dada por

$$
\phi_{X}(t)=e^{-a|t|}
$$


3.7.7.2 - Verificação de que a função geradora de momentos para a distribuição de Cauchy não existe

Tem-se, por definição, que

$$
\begin{aligned}
m_{X}(t) & =E\left[e^{t x}\right]=E\left[e^{t x} \frac{a}{\pi\left(x^{2}+a^{2}\right)}\right] \\
& =\frac{a}{\pi} \int_{-\infty}^{+\infty} \frac{e^{t x}}{x^{2}+a^{2}} d x
\end{aligned}
$$

que não existe para $\underline{t}$ real. Com efeito, basta notar, por exemplo, que se $x>0, t>0$,

$$
e^{t x}=1+t x+\frac{t^{2} x^{2}}{2 !}+\frac{t^{3} x^{3}}{3 !}+\ldots>\frac{t^{2} x^{2}}{2 !}
$$

de modo que

$$
\frac{a}{\pi} \int_{-\infty}^{+\infty} \frac{e^{t x}}{x^{2}+a^{2}} d x \geq \frac{a}{\pi} \frac{t^{2}}{2} \int_{0}^{\infty} \frac{x^{2}}{x^{2}+a^{2}} d x
$$

ond e $\int_{0}^{\infty} \frac{x^{2}}{x^{2}+a^{2}} d x$ diverge.

Demonstra-se, então, que a integral

$$
\int_{0}^{\infty} \frac{x^{2}}{x^{2}+a^{2}} d x
$$

diverge. 
164.

$$
\text { Em } \begin{aligned}
\int_{0}^{\infty} \frac{x^{2}}{x^{2}+a^{2}} d x, & \text { substituindo-se } \\
x^{2}+a^{2} & =a^{2} \sec ^{2} \theta \\
x & =a \operatorname{tg} \theta \\
d x & =a \sec ^{2} \theta d \theta
\end{aligned}
$$

tem-se

$$
\begin{aligned}
\int_{0}^{\pi / 2} \frac{a^{2} \operatorname{tg}^{2} \theta}{a^{2} \sec ^{2} \theta} a \sec ^{2} \theta d \theta & =a \int_{0}^{\pi / 2} \operatorname{tg}^{2} \theta d \theta \\
& =a \int_{0}^{\pi / 2}\left(\sec ^{2} \theta-1\right) d \theta \\
& =a[\operatorname{tg} \theta-\theta]_{0}^{\pi / 2} \\
& =\infty
\end{aligned}
$$

de forma que $\int_{0}^{\infty} \frac{x^{2}}{x^{2}+a^{2}} d x$ diverge.

Alëm disso, verifica-se que tambēm para $t<0, m_{X}(t)$

não existe. Dessa forma, apenas para $t=0, m_{X}(t)$ existe, ou seja,

$$
m_{X}(0)=E\left[e^{0}\right]=1
$$




\subsubsection{3 - Obtenção da função característica de uma distribuição de Cauchy}

Tem-se, por definição, que:

$$
\begin{aligned}
\phi_{X}(t) & =E\left[e^{i t x}\right]=E\left[e^{i t x} \frac{a}{\pi} \frac{1}{\left(x^{2}+a^{2}\right)}\right] \\
& =\frac{a}{\pi} \int_{-\infty}^{+\infty} \frac{e^{i t x}}{x^{2}+a^{2}} d x \\
& =\frac{a}{\pi} \int_{-\infty}^{+\infty} \frac{\cos (t x)+i \operatorname{sen}(t x)}{a^{2}+x^{2}} d x \\
& =\frac{a}{\pi} \int_{-\infty}^{+\infty} \frac{\cos (t x)}{a^{2}+x^{2}} d x+i \frac{a}{\pi} \int_{-\infty}^{+\infty} \frac{\operatorname{sen}(t x)}{a^{2}+x^{2}} d x \\
& =\frac{2 a}{\pi} \int_{0}^{\infty} \frac{\cos (t x)}{a^{2}+x^{2}} d x \\
& =\frac{2 a}{\pi} \frac{\pi}{2 a} e^{-a|t|} \\
& =e^{-a|t|}
\end{aligned}
$$

pois,

$$
\int_{-\infty}^{+\infty} \frac{\operatorname{sen}(t x)}{a^{2}+x^{2}} d x=0 \quad \text { e } \quad \int_{0}^{\infty} \frac{\cos (t x)}{a^{2}+x^{2}}=\frac{\pi}{2 a} e^{-a|t|}
$$

como se demonstra a seguir. 
Considere-se inicialmente que

$$
\oint_{\mathbb{C}} \frac{e^{i t z}}{z^{2}+a^{2}} d z
$$

onde $\mathbb{C}$ è caminho do lema 2 (Apêndice).

0 integrando possui polos simples em $z= \pm$ ai, dos quais somente $z=$ ai é interior a $\mathbb{C}$.

0 resíduo em $z=a i \quad \bar{e}$

$$
\lim _{z \rightarrow a i}\left[(z-a i) \frac{e^{i t z}}{(z-a i)(z+a i)}\right]=\frac{e^{-a t}}{2 a i}
$$

Então,

$$
\oint_{\mathbb{C}} \frac{e^{i t z}}{z^{2}+a^{2}} d z=2 \pi_{i}\left(\frac{e^{-a t}}{2 a i}\right)=\frac{\pi}{a} e^{-a t}
$$

pelo teorema dos resíduos (Apêndice).

$$
\text { Daí, }
$$

$$
\int_{-R}^{R} \frac{e^{i t x}}{x^{2}+a^{2}} d x+\int_{\Gamma} \frac{e^{i t z}}{z^{2}+a^{2}}=\frac{\pi}{a} e^{-a t}
$$

ou

$$
\int_{-R}^{R}\left[\frac{\cos (t x)+i \operatorname{sen}(t x)]}{x^{2}+a^{2}}\right] d x+\int_{\Gamma} \frac{e^{i t z}}{z^{2}+a^{2}} \cdot d z=\frac{\pi}{a} e^{-a t}
$$




$$
\int_{-R}^{R} \frac{\cos (t x)}{a^{2}+x^{2}} d x+i \int_{-R}^{R} \frac{\operatorname{sen}(t x)}{a^{2}+x^{2}} d x+\int_{\Gamma} \frac{e^{i t z}}{z^{2}+a^{2}} d z=\frac{\pi}{a} e^{-a t}
$$

$2 \int_{0}^{R} \frac{\cos (t x)}{a^{2}+x^{2}} d x+i \int_{-R}^{R} \frac{\operatorname{sen}(t x)}{a^{2}+x^{2}} d x+\int_{\Gamma} \frac{e^{i t z}}{z^{2}+a^{2}} d z=\frac{\pi}{a} e^{-a t}$

e, considerando-se o lema 2 (Apêndice), como $F(z)=\frac{1}{z^{2}+a^{2}}$ è limita da no círculo $z=\operatorname{Re}^{i \theta}$, tem-se que

$$
\int \frac{e^{i t z}}{z^{2}+a^{2}}=0
$$

de forma que

$$
2 \int_{0}^{R} \frac{\cos (t x)}{a^{2}+x^{2}} d x+i \int_{-R}^{R} \frac{\operatorname{sen}(t x)}{a^{2}+x^{2}} d x=\frac{\pi}{a} e^{-a t}
$$

e, aplicando-se o limite para $R$ tendendo a infinito $(R \rightarrow \infty)$, tem-se que:

$$
\int_{0}^{\infty} \frac{\cos (t x)}{a^{2}+x^{2}} d x=\frac{\pi}{2 a} e^{-a t}
$$

e

$$
\int_{-\infty}^{+\infty} \frac{\operatorname{sen}(t x)}{a^{2}+x^{2}} d x=0
$$

e, como a função cosseno è par, pode-se trabalhar com $|t|$, de forma que

$$
\int_{0}^{\infty} \frac{\cos (t x)}{x^{2}+a^{2}} d x=\frac{\pi}{2 a} e^{-a|t|}
$$


168.

$$
\begin{aligned}
\text { Como } \phi_{X}(t) & =e^{-a|t|} \\
& \frac{d}{d t} \phi_{X}(t)=\frac{d}{d t}\left[e^{-a|t|]}\right.
\end{aligned}
$$

que não existe para $t=0$. Dessa forma, não existe $\mu_{1}^{\prime}$ e qualquer outro $\mu_{r}^{\prime}$ como se afirma em (3.7.7.1). 
169.

4. RESULTADOS E DISCUSSOES

Embora esta dissertação seja essencialmente teórica, por se tratar de uma revisão bibliogräfica, alguns resultados impor tantes devem ser salientados, bem como as generalizações teóricas comprovadas.

4.1 - Definição de Momentos

Quanto à definição geral de momentos, salienta-se a fórmula que se constitui na relação geral entre momentos centrados e momentos em relação à origem, pois a mesma é um instrumento de gran de utilidade para obtençáo de momentos de ordem elevada a partir do conhecimento dos momentos de ordem mais baixa, embora o trabalho al gébrico possa ser considerado exaustivo. Porém, como em termos prá- 
ticos, para caracterização de uma distribuição, dificilmente se pre cisa obter momentos de ordem superior à quarta, basta que se saiba que as igualdades:

$$
\begin{aligned}
& \mu_{2}^{\prime}=\mu_{2}+\mu_{1}^{2} \\
& \mu_{3}^{\prime}=\mu_{3}+3 \mu_{2} \mu_{1}^{\prime}+\mu_{1}^{3} \\
& \mu_{4}^{\prime}=\mu_{4}+4 \mu_{3} \mu_{1}^{\prime}+6 \mu_{2} \mu_{1}^{2}+\mu_{1}^{4}
\end{aligned}
$$

e

$$
\begin{aligned}
& \mu_{2}=\mu_{2}^{\prime}-\mu_{1}^{2} \\
& \mu_{3}=\mu_{3}^{\prime}-3 \mu_{1}^{\prime} \mu_{2}^{\prime}+2 \mu_{1}^{3} \\
& \mu_{4}=\mu_{4}^{\prime}-4 \mu_{1}^{\prime} \mu_{3}^{\prime}+6 \mu_{1}^{2} \mu_{2}^{\prime}-3 \mu_{1}^{4}
\end{aligned}
$$

são obtidas pelo desenvolvimento algëbrico de

$$
\mu_{r}^{\prime}=\sum_{j=0}^{r}\left(\begin{array}{l}
r \\
j
\end{array}\right) \mu_{r-j} \mu_{1}^{j}=\left(\mu+\mu_{l}^{\prime}\right)^{r}
$$

\section{2 - Funções Geradoras de Momentos}

Destacam-se, neste trabalho, duas principais funções geradoras de momentos, a função geradora de momentos,

$$
m_{X}(t)=E\left[e^{t x}\right]=\sum_{j=0}^{\infty} \frac{1}{j !} \mu_{j}^{!} t^{j},
$$

e a função característica,

$$
\phi_{X}(t)=E\left[e^{i t x}\right]=\sum_{j=0}^{\infty} \frac{1}{j !} \mu_{j}^{\prime}(i t)^{j}
$$


Além disso, faz-se referência à função geradora de momentos fatorial, $\eta_{X}(t)=E\left(t^{X}\right)$, de utilidade praticamente restrita ao caso discreto, como ao Binomial e Poisson para momentos até $2^{a}$ ordem.

Uma vantagem da função característica sobre a função geradora de momentos é o fato de que ela sempre existe, ao passo que a f.g.m. pode não existir, como ocorre no caso da distribuição de Cauchy. Observa-se, entretanto, que se $m_{X}(t)$ existe, $\phi_{X}(t)$ é cor respondente a $\mathrm{m}_{\mathrm{X}}(\mathrm{it})$, de forma que a obtenção dos momentos $\bar{e}$ mais simples algebricamente se feita através de $\mathrm{m}_{X}(t)$, por não ser neces sário trabalhar no campo complexo.

Apresentam-se, condensadamente, nas Tabelas 1 e 2 , as funções geradoras de momentos obtidas no decorrer deste trabalho, onde evidencia-se que, se $m_{X}(t)$ existe, $\phi_{X}(t)=m_{X}(i t)$, e também os principais momentos que caracterizam uma distribuição (média e variâncià).

\section{3 - General ização da Fōrmula Recorrente (NOGUEIRA, 1965)}

Aplicando-se a förmula recorrente (NOGUEIRA, 1965) às distribuições Bernoulli, Geométrica, Binomial Negativa, Exponencial (negativa) e Gama, obteve-se, para a mesma, uma generalização do que jä estava assentado para as distribuições Binomial, Poisson e Normal. Resume-se, na Tabela 3, o resultado dessa generalização. 
172.

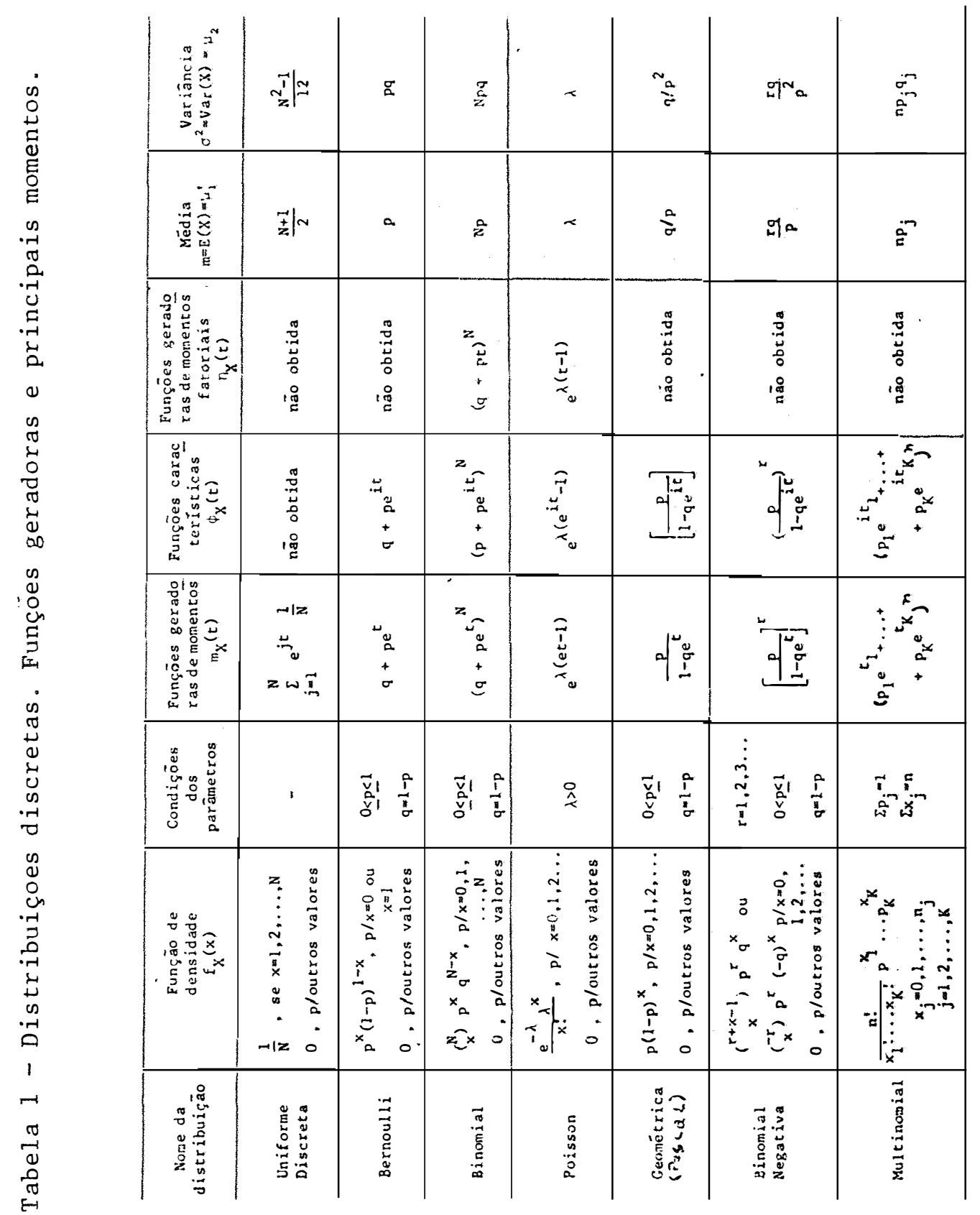


173.

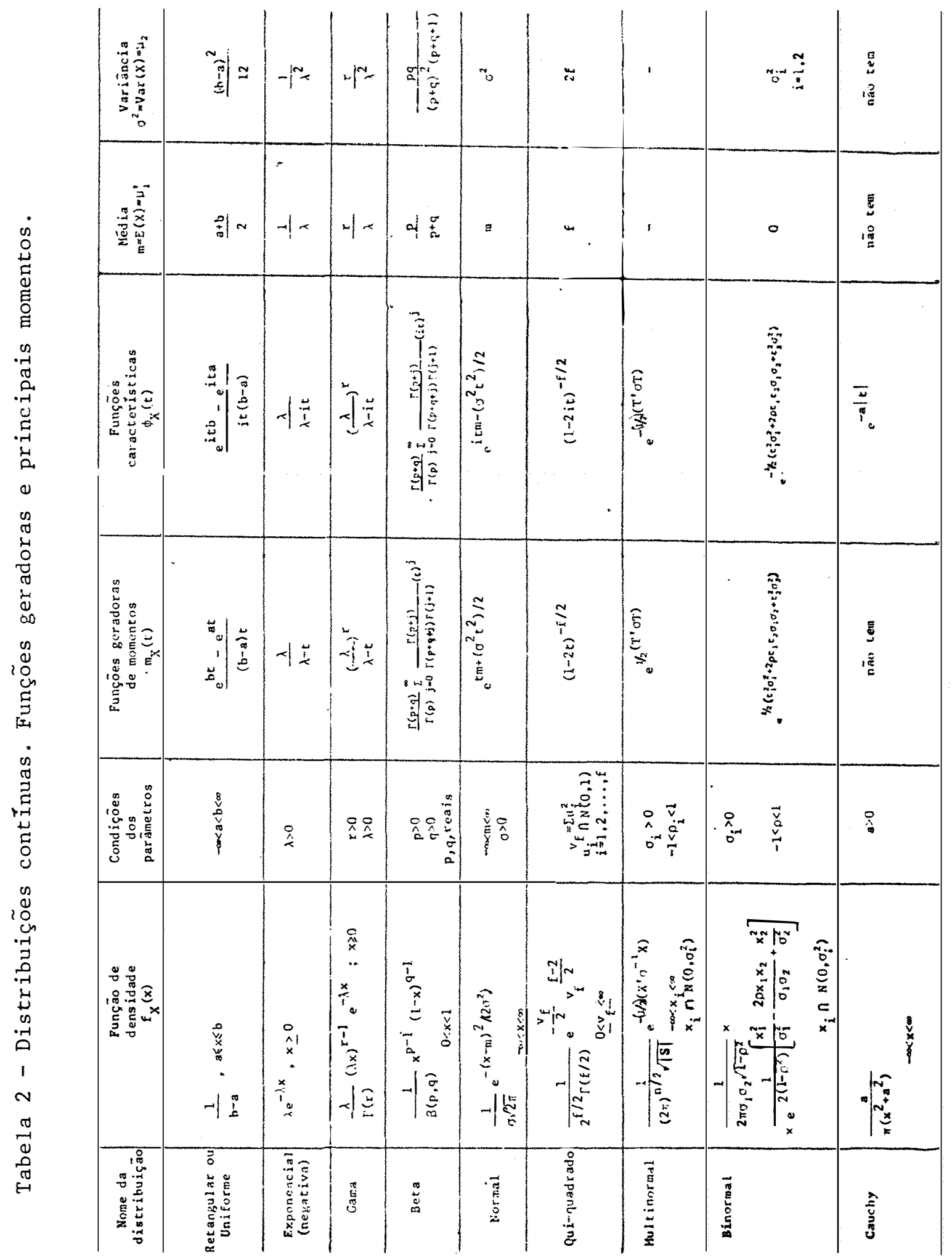


Tabela 3 - Förmula recorrente (NOGUEIRA, 1965) e generalizações.

$$
\text { Se } \frac{d}{d m}\left[f_{X}(x)\right]=\left[\frac{x-m}{\sigma^{2}}\right] f_{X}(x) \text {, então, } \mu_{n+1}=\sigma^{2} \frac{d}{d m}\left(\mu_{n}\right)+n \sigma^{2} \mu_{n-1}
$$

Distribuição

$$
\mu_{n+1}=\sigma^{2} \frac{d}{d m}\left(\mu_{n}\right)+n \sigma^{2} \mu_{n-1}
$$

Bernoulli

$$
p q \frac{d}{d m}\left(\mu_{n}\right)+n p q \mu_{n-1}
$$

Binomial (NOGUEIRA, 1965) Npq $\frac{d}{d m}\left(\mu_{n}\right)+n$ Npq $\mu_{n-1}$

$$
\text { Poisson (NOGUEIRA, 1965) } \lambda \frac{d}{d m}\left(\mu_{n}\right)+n \lambda \mu_{n-1}
$$

Geométrica (Pascal) $\frac{\mathrm{q}}{\mathrm{p}^{2}} \frac{\mathrm{d}}{\mathrm{dm}}\left(\mu_{\mathrm{n}}\right)+\mathrm{n} \frac{\mathrm{q}}{\mathrm{p}^{2}} \mu_{n-1}$

Binomial Negativa $\quad \frac{\mathrm{rq}}{\mathrm{p}^{2}} \frac{\mathrm{d}}{\mathrm{dm}}\left(\mu_{\mathrm{n}}\right)+\mathrm{n} \frac{\mathrm{rq}}{\mathrm{p}^{2}} \mu_{\mathrm{n}-1}$

Exponencial (negativa) $\frac{1}{\lambda^{2}} \frac{d}{d m}\left(\mu_{n}\right)+n \frac{1}{\lambda^{2}} \mu_{n-1}$

Gama

$$
\frac{r}{\lambda^{2}} \frac{d}{d m}\left(\mu_{n}\right)+n \frac{r}{\lambda^{2}} \mu_{n-1}
$$

Normal (NOGUEIRA, 1965) $\sigma^{2} \frac{d}{d m}\left(\mu_{n}\right)+n \sigma^{2} \mu_{n-1}$ 


\section{4 - Técnica da Função Geradora de Momentos}

A maior importância prática do estudo das funções ge radoras de momentos do tipo $\mathrm{m}_{X}(t)$ não reside, simplesmente, no fato de que elas geram momentos, mas sim na aplicação da técnica da função geradora de momentos que se apresenta em 3.6. Esta afirmação é feita em função dessa técnica permitir que:

i) se obtenham distribuições conjuntas, desde que sejam conhecidas as distribuições de probabilidade das variáveis aleatórias que a definem, o que se constitui como uma ferramenta de grande utilidade em teoria estatística aplicada, embora a mesma seja limitada para $k>1$, pelo fato de que pou cas funções geradoras de momentos conjuntos são de imediato reconheciveis. Porém, para $k=1$, è de extrema utilidade;

ii) seja possível identificar a distribuição da soma de variāveis aleatōrias independentes, tambēm de importância fundamental em estatistica aplicada;

iii) se encontrem distribuições limites, também de grande importância em estatística aplicada, o que permite a utilização da distribuição limite como uma aproximação para a exata fun ção de distribuição. Isto é extremamente útil para o caso em que as tabelas de probabilidade se limitam a $\underline{n}$ pequeno para uma determinada variável X com distribuição de probabị 
lidade conhecida, se essa variāvel X tem por distribuição lí mite de probabilidade (para $\underline{\mathrm{n}}$ grande) uma distribuição cuja tabela independe de $\underline{n}$, tipo normal padrão. Além disso, por permitir que se prove a forma especial do teorema do limite central, apesar da limitação imposta de que se deve admitir a existência de uma função geradora de momentos para a distribuição da qual se extrai a amostra.

Outros resultados da aplicação da técnica da função geradora de momentos, além dos que se desenvolvem nesta dissertação, podem ser pesquisados. Resume-se, na Tabela 4,0 que se desenvolve nes te trabalho como aplicação desta técnica.

\section{5 - Momentos da Distribuição Normal}

Em uma distribuição normal, o cálculo de momentos é extremamente simples, pois todos os momentos em relação à média, de ordem İmpar, são nulos, e os de ordem par são dados pela fórmula re corrente que se resume na Tabela 5. Os momentos em relação à origem são facilmente obtidos pela relação geral entre os momentos que se apresenta em 3.2.7. 
Tabela 4 - Aplicações da técnica da função geradora de momentos.

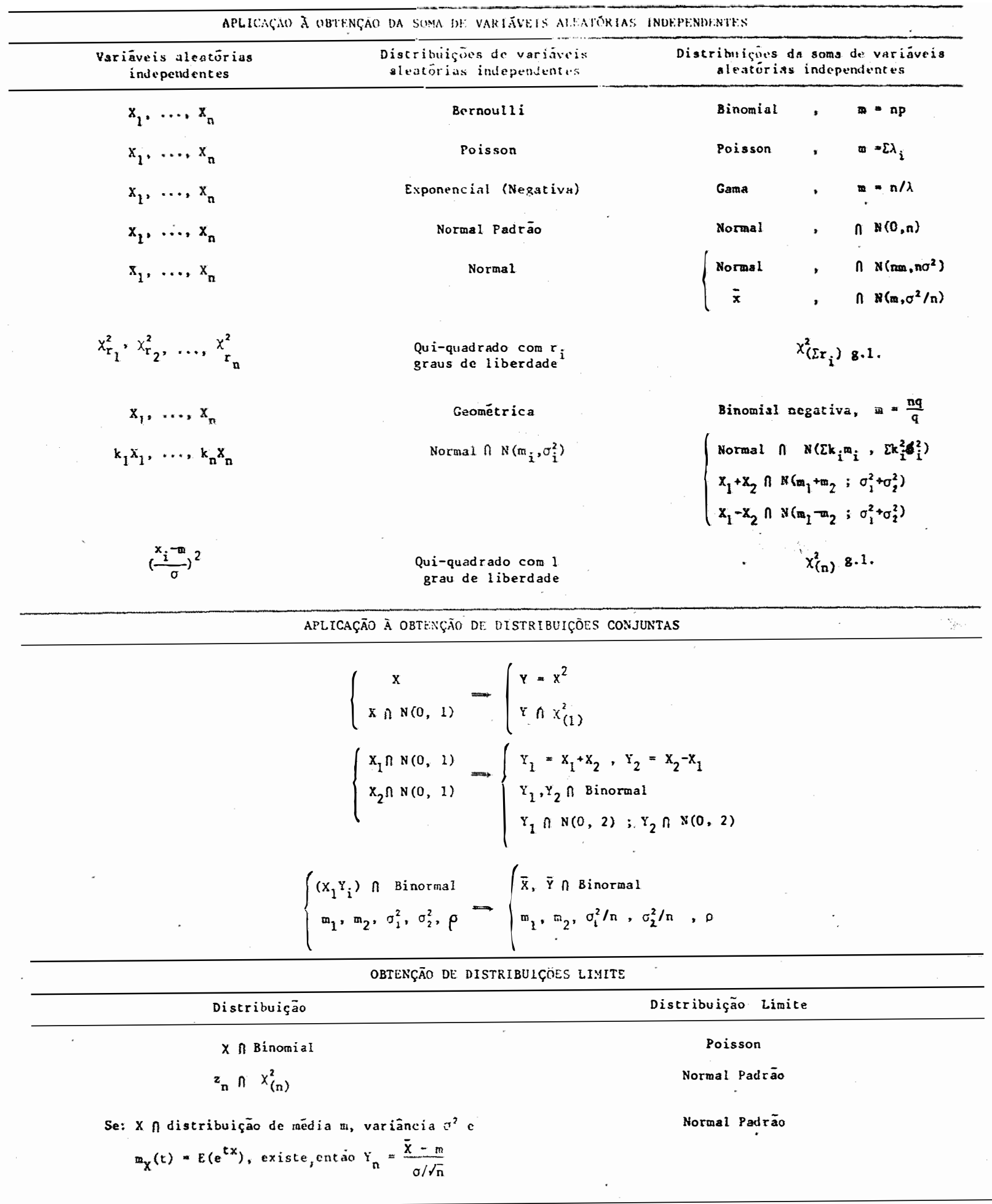


Tabela 5 - Distribuição Normal. Fórmula recorrente para momentos de ordem par.

$\begin{array}{rlrl} & x \cap N(0,1) & x \cap N\left(m, \sigma^{2}\right) \\ \mu_{2 n+1}=0 & \mu_{2 n+1}=0 \\ \mu_{2 n}=(2 n-1) \mu_{2 n-2} & \mu_{2 n}=(2 n-1) \sigma^{2} \mu_{(2 n-2)} \\ =\frac{(2 n) !}{2^{n}(n) !} & =\frac{(2 n) !}{2^{n}(n) !} \sigma^{2 n}\end{array}$

\section{6 - Teorema da Inversão}

A principal razão de se apresentar um capítulo deste trabalho dedicado ao estudo das funções características não se deve ao fato de que essas funções são geradoras de momentos que sempre existem, pois as distribuições de probabilidade mais utilizadas em estatística aplicada possuem funções geradoras de momentos do tipo $\mathrm{m}_{\mathrm{X}}(\mathrm{t})$ como se apresenta em 3.4, e distribuições tipo Cauchy, que não possuem $m_{X}(t)$, mas $\operatorname{sim} \phi_{X}(t)$, são raramente utilizadas. A razão fun damental, então, de se estudar a teoria das funções características é o conhecimento do teorema da inversão, porque ele permite que se determine a função de densidade de uma variável aleatöria X, a partir de sua função característica, como se resume na Tabela 6 . 
179.

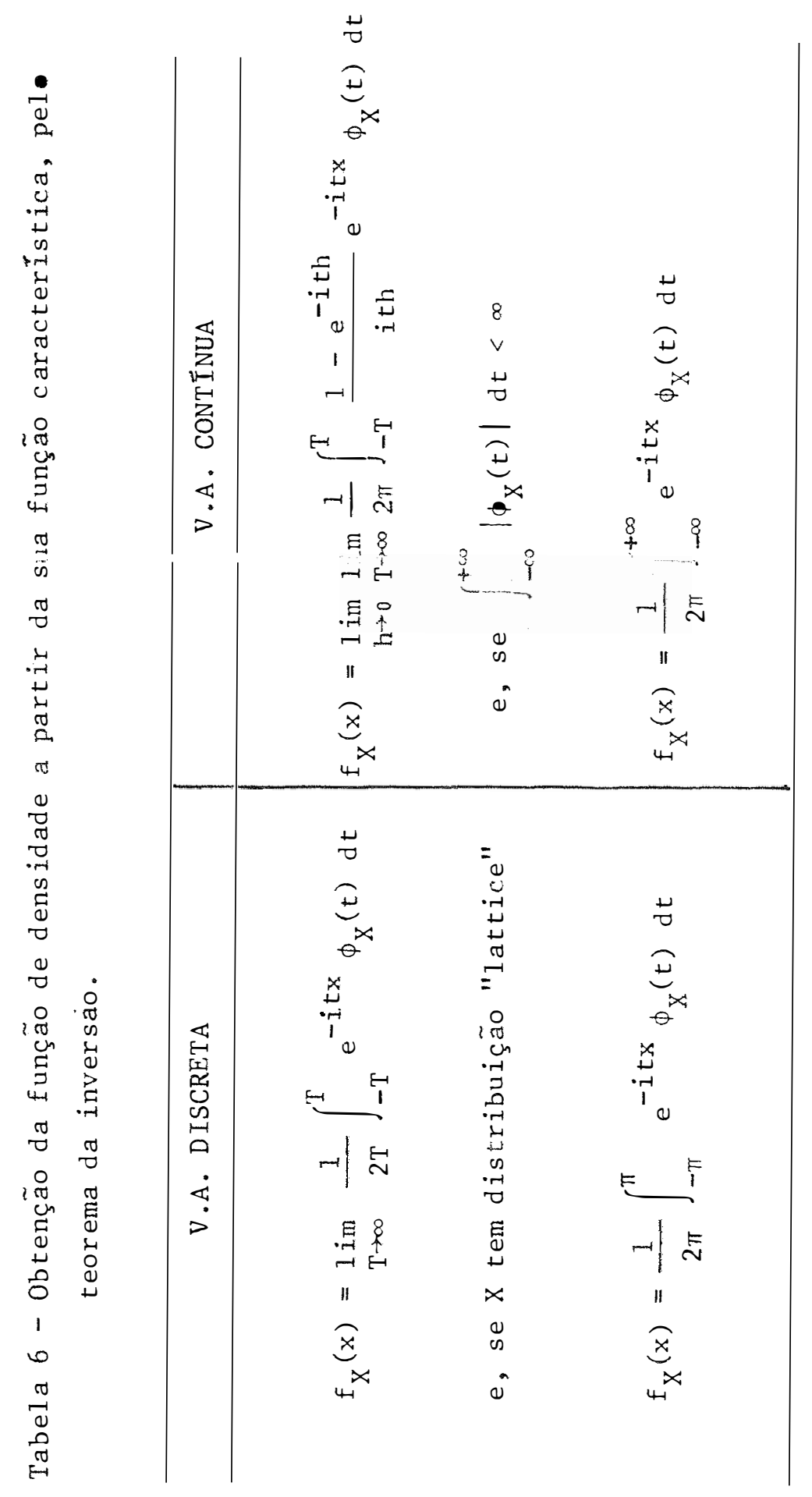


180.

\section{CONCLUSOES}

- Os momentos de uma distribuição são obtidos pelas próprias de finições, ou por funções geradoras de momentos, dos tipos $m_{X}(t)=E\left(e^{t X}\right)$ se a esperança existe, ou $\phi_{X}(t)=E\left[e^{i t x}\right]$ que sempre existe.

- Existindo $m_{X}(t), \quad \phi_{X}(t)=m_{X}(i t)$.

- A função $m_{X}(t)$ é mais simples de ser operada algebricamente que a função $\phi_{X}(t)$.

- A função geradora de momentos pode não existir, porëm, existe para as distribuições mais usuais da Estatística.

- Os momentos de ordem elevada nas distribuições Bernoulli, Bí nomial, Poisson, Geométrica, Binomial Negativa, Exponencial 
(negativa), Gama e Normal são imediatamente obtidos através da generalização da fórmula recorrente.

- Distribuições conjuntas, distribuições da soma de variáveis aleatōrias independentes, e distribuiçoes limites podem ser encontradas pela utilizaçào da técnica da função geradora de momentos .

- Aplica-se uma fórmula recorrente para o cálculo dos momentos de ordem par em uma distribuição normal.

- Obtêm-se funções de densidade pela aplicação do teorema da inversão às suas funções características. 


\section{LITERATURA CITADA}

CHURCHILL, R.V., 1975. Variāveis Complexas e Suas Aplicações. São Paulo, McGraw Hill do Brasil e Editora da USP. 276 p.

CRÄMR, H., 1958. The Elements of Probability Theory. 4. edição, Nova York, John Wiley. 281 p.

CRAMER, H., 1974. Mathematical Methods of Statistics. 3. edição, Princeton, Princeton University Press. 575 p.

CRUZ, H.V., 1980. Emprego da Função Fatorial para o Cálculo de Momentos de uma Distribuição de Frequência. Revista Brasileira de. Estatistica, Fundação IBGE, Rio de Janeiro, 162: 267-287.

DIXON, J.W. e F.J. MASSEY Jr., 1969. Introduction to Statistical Analysis. 3 . edição, Tóquio, International Student Edition (Mc Graw-Hill Koga Kusha). 638 p.

HOEL, P.G., 1980. Estatística Matemática. 4. edição, Rio de Janei ro, Editora Guanabara Dois. 373 p. 
HOGG, R.V. e A. CRAIG, 1970. Introduction to Mathematical Statistics. 3. edição, Nova York, Macmillan. 415 p.

KENDALL, M. e A. STUART, 1977. The Advanced Theory of Statistics. Vol. 1. 4. edição, Nova York, Macmillan. 472 p.

LU KACS, E., 1970. Characteristic Functions. 2. edição, Londres, Charles Griffin. 350 p.

MOOD, A.M.; F.A. GRAYBILL e D.C. BOES, 1974. Introduction to the Theory of Statistics. 3. edição, Tóquio, International student Edition (McGraw-Hill Koga Kusha). 564 p.

NOGUEIRA, I.R., 1965. Förmula Geral para Obtenção dos Momentos nas Distribuições Binomial, Poisson e Normal. Boletim Didático, ESALQ/USP, Piracicaba, no 12.

NOGUE IRA, I.R., 1968. Aplicação de Matrizes no Estudo da Distribuiçāo Multinormal. Revista Ciência e Cultura. São Paulo, 20(3): 601-611.

PIEDRABUENA, A.E., 1968. Distribuições Interferidas - Estudo Sobre uma Distribuição Generalizada. Piracicaba, ESALQ/USP. 65 p. (Dissertaçāo de Mestrado).

ROUSSAS, G.G., 1973. A First Course in Mathematical Statistics. Wisconsin, Addison-Wesley. $506 \mathrm{p}$.

SPIEGEL, M.R., 1971. Cälculo Avançado. Säo Paulo, McGraw-Hill. $500 \mathrm{p}$. 
SPIEGEL, M.R., 1971. Variāveis Complexas. São Paulo, McGraw-Hill. $468 \mathrm{p}$.

SPIEGEL, M.R., 1978. Probabilidade e Estatística. São Paulo, Mc-Graw Hill. 518 p.

TATON, R. (Coord.), 1964. La Science Contemporaine. In: HISTOIRE GÉNERALE DES CIENCES, Vol. II. Paris, Presses Universitaires de France. 1080 p.

TORANZOS, F.I., 1971. Teoria Estadística y Aplicaciones. 3. edição, Buenos Aires, Editorial Kapelusz. 448 p.

WILKS, S.S., 1962. Mathematical Statistics. Nova York, John Wiley. $644 \mathrm{p}$. 


\section{APENDICE}

\section{1 - Teorema do Limite Central (SPIEGEL, 1978)}

Sejam $x_{1}, x_{2}, \ldots$, variāveis aleatórias independentes, identicamente distribuỉdas e com média m e variância $\sigma^{2}$ finitas. Então, se

$$
\begin{array}{r}
s_{n}=x_{1}+x_{2}+\ldots+x_{n} \quad(n=1,2, \ldots) \\
\lim _{n \rightarrow \infty} P\left(a \leq \frac{s_{n}-n m}{\sigma \sqrt{n}} \leq b\right)=\frac{1}{\sqrt{2 \pi}} \int_{a}^{b} e^{-u^{2} / 2} d u
\end{array}
$$

is to é, a variāvel aleatōria $\left(S_{n}-n m\right) /(\sigma \sqrt{n})$, que $\bar{e}$ a variāvel padronizada correspondente a $S_{n}$, $\bar{e}$ assintoticamente normal.

o teorema é válido também sob condições mais gerais, por exemplo, quando $x_{2}, x_{2}, \ldots$, são variāveis aleatórias indepen- 
dentes com a mesma média e a mesma variância, mas não necessariamen te com a mesma distribuição.

\section{2 - Função Gama}

$$
\Gamma(\alpha+1)=\int_{0}^{\infty} x^{\alpha} e^{-x} d x \quad, \quad \alpha>-1
$$

PROPRIEDADE: $\Gamma(\alpha+1)=\alpha \Gamma(\alpha)$.

\section{3 - Função Beta}

$$
\beta(a, b)=\int_{0}^{1} x^{a-1}(1-x)^{b-1} d x,\left\{\begin{array}{l}
a>0 \\
b>0
\end{array}\right.
$$

ou

$$
\begin{aligned}
& \qquad(a, b)=2 \int_{0}^{\pi / 2}(\operatorname{sen} \theta)^{2 a-1}(\cos \theta)^{2 b-1} d \theta \\
& \text { PROPRIEDADE: } \beta(a, b)=\frac{\Gamma(a) \Gamma(b)}{\Gamma(a+b)}
\end{aligned}
$$

7.4 - Série de MacLaurin

$$
f(x)=f(0)+f^{\prime}(0) \frac{x}{1 !}+f^{\prime \prime}(0) \frac{x^{2}}{2 !}+\ldots+f^{n}(0) \frac{x^{n}}{n !}+\cdots
$$




$$
f(x)=f(a)+f^{\prime}(a) \frac{x-a}{1 !}+f^{\prime \prime}(a) \frac{(x-a)^{2}}{2 !}+\ldots+f^{n-1}(a) \frac{(x-a)^{n-1}}{(n-1) !}+R
$$

onde $R=f^{n}\left(x_{1}\right) \cdot \frac{(x-a)^{n}}{n !},\left(a<x_{1}<x\right)$.

o termo $\mathrm{R}$ diz-se o resto depois do n-egësimo termo.

7.6 - Binômio de Newton

$$
\begin{aligned}
(x+a)^{n}=\left(\begin{array}{l}
n \\
0
\end{array}\right) a^{0} x^{n} & +\left(\begin{array}{l}
n \\
1
\end{array}\right) a x^{n-1}+\left(\begin{array}{l}
n \\
2
\end{array}\right) a^{2} x^{n-2}+ \\
& +\ldots+\left(\begin{array}{l}
n \\
n
\end{array}\right) a^{n} x^{0} \\
= & \sum_{j=0}^{n}\left(\begin{array}{l}
n \\
j
\end{array}\right) a^{j} x^{n-j}
\end{aligned}
$$

7.7 - Distribuição "Lattice"

Se $X$ è uma variävel aleatōria que possa ser escrita na forma $x=a+k h$, com $\underline{a}, \underline{h}$, constantes, $h>0$ e $\underline{k}$ variando no conjunto $0,1,2, \ldots, n$, ou $0,1,2, \ldots$ ou $0, \pm 1, \pm 2, \ldots, \pm n$, ou $0, \pm 1, \pm 2, \pm 3, \ldots$, chama-se a distribuição de $X$ de "distribuição lattice".

Exemplificando, se $\mathrm{x}$ tem distribuiçāo binomial, então os valores de $x$ são da forma $x=a+k h, \operatorname{com} a=0, h=1, e k=0,1$, $2, \ldots, n$. 
Se $\phi_{X}(t)$ é a função característica de $X$, pode-se mos trar que a distribuição de X é uma "distribuiçäo lattice", se e somente se, $\left|\Phi_{X}(t)\right|=1$ para algum $t \neq 0$ (ROUSSAS, 1973). Pode tambëm ser mostrado que a distribuiçào de x é uma "distribuição lattice", se e somente se, a função característica $\phi_{X}(t)$ é periódica com período $2 \pi$ (isto é, $\left.\phi_{X}(t+2 \pi)=\phi_{X}(t), t \in \mathbf{R}\right)$ (Ro USSAS, 1973).

\section{8 - Lema (1):}

A integral $\int_{0}^{x} \frac{\text { sen } y}{y}$ dy é 1imitada para $x>0 e$ e aproxima-se de $\pi / 2$ com $x$ tendendo a infinito (LUKACS, 1970).

7.9 - Lema (2):

Se $|F(z)| \leq M / R^{K}$, para $z=R e^{i \theta}$, onde $K>0$ e $M=$ cte, então $\lim _{R \rightarrow \infty} \int_{\Gamma} e^{i t z} F(z) d z=0$, onde $\Gamma \vec{e} \circ$ semi-círculo do semi-plano $\operatorname{Imz} \geq 0$, de raio $R$ e centro na origem.

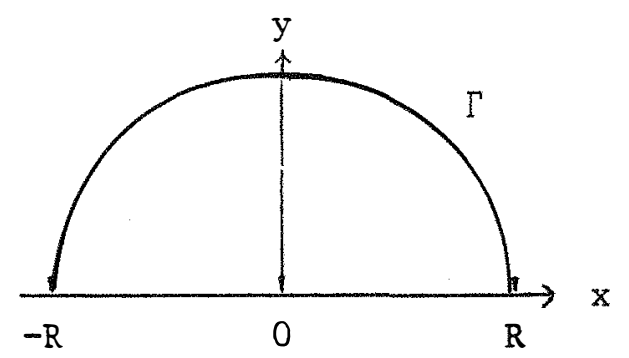


Demonstração:

$$
\begin{aligned}
& \text { Se } \quad z=\operatorname{Re}^{i \theta} \text {, } \\
& \int_{\Gamma} e^{i t z} F(z) d z=\int_{0}^{\pi} e^{i t} \operatorname{Re} F\left(R e^{i \theta}\right) i R e^{i \theta} d \theta \\
& \left|\int_{0}^{\pi} e^{i t} \operatorname{Re}^{i \theta} F\left(R e^{i \theta}\right) i R e^{i \theta} d \theta\right| \leq \int_{0}^{\pi}\left|e^{i t} \operatorname{Re} F\left(R e^{i \theta}\right) i R e^{i \theta}\right| d \theta= \\
& =\int_{0}^{\pi}\left|e^{i t R \cos \theta-t R \operatorname{sen} \theta} F\left(R e^{i \theta}\right) i R e^{i \theta}\right| d \theta= \\
& =\int_{0}^{\pi} e^{-t R \operatorname{sen} \theta}\left|F\left(R e^{i \theta}\right)\right| R d \theta \leq \frac{M}{R^{K-1}} \int_{0}^{\pi} e^{-t R \operatorname{sen} \theta} d \theta \\
& =\frac{2 M}{R^{K-1}} \int_{0}^{\pi / 2} e^{-t R \operatorname{sen} \theta} d \theta \\
& \text { Para } 0 \leq \theta \leq \pi / 2 \text {, tem-se sen } \theta \geq 2 \theta / \pi \text {, logo, } \\
& \frac{2 M}{R^{K-1}} \int_{0}^{\pi / 2} e^{-t R \operatorname{sen} \theta} d \theta \leq \frac{2 M}{R^{K-1}} \int_{0}^{\pi / 2} e^{-(2 t R \theta) / \pi} d \theta= \\
& =\frac{-2 M}{R^{K-1}} \int_{0}^{\pi / 2} e^{u} \frac{\pi}{2 t R} d u=\left[\frac{-M \pi}{t R^{K}} e^{-(2 t R \theta) / \pi}\right]_{0}^{\pi / 2}= \\
& =\frac{-M \pi}{t R^{K}}\left(e^{-t R}-1\right)=\frac{M \pi}{t R^{K}}\left(1-e^{-t R}\right),
\end{aligned}
$$

que tende a zero quando $R$ tende a infinito. 
190.

7.10 - Limite Fundamental (1) (HOOG e CRAIG, 1970)

$$
\lim _{n \rightarrow \infty}\left[1+\frac{b}{n}+\frac{\psi(n)}{n}\right]^{c n}=\lim _{n \rightarrow \infty}\left(1+\frac{b}{n}\right)^{c n}=e^{b c}
$$

onde $\underline{b}$ e $\underline{c}$ não dependem de $\underline{\mathrm{n}}$, e $\lim _{\mathrm{n} \rightarrow \infty} \psi(\mathrm{n})=0$.

7.11 - Teorema dos Resîduos (Cauchy) - (SPIEGEL, 1973)

"Se $\underline{f}$ é analítica e univalente numa região $R$, exceto em um número finito de singularidades isoladas $z_{1}, z_{2}, \ldots, z_{k}$, então,

$$
\int_{C} f(z) d z=2 \pi \underset{i}{\sum_{j=1}^{K}}(\text { res } f)\left(z_{j}\right)
$$

onde $\underline{C}$ é um contorno fechado de $R$, envolvendo $z_{1}, z_{2}, \ldots, z_{K}$, uma vez no sentido positivo." 